\title{
JG|U
}

Gutenberg School of Management and Economics \& Research Unit "Interdisciplinary Public Policy" Discussion Paper Series

\section{Bubbly Markov Equilibria}

Martin Barbie and Marten Hillebrand

March 2017

\section{Discussion paper number 1703}


Contact details:

Martin Barbie

CMR-Center for Macroeconomic Research

University of Cologne

Albertus-Magnus-Platz

50923 Köln, Germany

barbie@wiso.uni-koeln.de

Marten Hillebrand

School of Management \& Economics

Gutenberg University Mainz

Jakob Welder Weg 4

55128 Mainz, Germany

mhillebr@uni-mainz.de 


\title{
Bubbly Markov Equilibria*
}

\author{
Martin Barbie $^{\dagger} \quad$ Marten Hillebrand ${ }^{\ddagger}$
}

March 13, 2017

\section{Revised version}

\begin{abstract}
Bubbly Markov Equilibria (BME) are recursive equilibria on the natural state space which admit a non-trivial bubble. The present paper studies the existence and properties of BME in a general class of overlapping generations (OLG) economies with capital accumulation and stochastic production shocks. Using monotone methods, we develop a general approach to construct Markov equilibria and provide necessary and sufficient conditions for these equilibria to be bubbly. Our main result shows that a BME exists whenever the bubbleless equilibrium is Pareto inefficient either due to overaccumulation of capital or inefficient risksharing between generations.
\end{abstract}

JEL classification: C62, D51, E32

Keywords: Asset Bubbles, Stochastic OLG, Production, Markov Equilibria, Pareto Optimality.

${ }^{*}$ We would like to thank Tim Deeken, Tomoo Kikuchi, Herakles Polemarchakis, Clemens Puppe, Kevin Reffett, Caren Söhner, John Stachurski, and Klaus Wälde for helpful comments and discussions and two anonymous referees for their constructive and very helpful comments. We also thank seminar participants at various conferences including the 2013 SAET Conference in Paris, the 2013 Annual Meeting of the Verein für Socialpolitik in Düsseldorf, and the 2014 CEF Conference in Oslo.

${ }^{\dagger}$ CMR-Center for Macroeconomic Research, University of Cologne, Albertus-Magnus-Platz, D50923, Köln, Germany, email: barbie@wiso.uni-koeln.de

$\ddagger$ (correspondence) School of Management \& Economics, Gutenberg University Mainz, Jakob Welder Weg 4 D-55128 Mainz, Germany, email: mhillebr@uni-mainz.de 


\section{Introduction}

A bubble is an intrinsically worthless asset which trades at a positive price such as fiat money or a bond with infinite maturity that never pays any dividends. Bubbly equilibria in which bubbles occur as an equilibrium outcome in the presence of rational investors only exist if the equilibrium price system satisfies certain conditions. In the presence of infinite-lived investors, these conditions can only hold in the presence of frictions such as borrowing constraints. In models with overlapping generations of investors, however, bubbles can occur even in the absence of such frictions which makes this class an attractive framework to study the existence and properties of bubbly equilibria.

The general contribution of this paper is to conduct such a study in a stochastic overlapping generations model with production. To our knowledge, we are the first to offer a comprehensive study of this type. Specifically, we state explicit conditions for the existence of bubbly equilibria in terms of the pricing kernel and provide a recursive method to construct them. Our main existence condition takes the form of an inefficiency property of the bubbleless equilibrium which recovers several existing results in the literature as special cases.

The emergence of a bubble in our setup has two important macroeconomic effects. First, it affects the formation of capital by providing an alternative investment opportunity to investors. Second, it creates an additional insurance possibility which affects the risk sharing arrangements among consumers. With these features, our setup encompasses previous studies of bubbly equilibria in OLG models for the case of a deterministic production economy as studied in Tirole (1985) as well as stochastic models with pure exchange as in Manuelli (1990), Aiyagari \& Peled (1991), or Magill \& Quinzii (2003). By construction, these studies either neglect the investment or the risk sharing effect of bubbles. In this sense, our framework contains these models as special cases and we will discuss which role the previous existence results play in our extended setup.

The stochastic OLG model with production has been studied in Wang $(1993,1994)$ and, more recently, in Morand \& Reffett (2007), McGovern et al. (2013), and Hillebrand (2014). These studies focus on a particular class of equilibria in which the equilibrium variables are determined by time-invariant mappings on the minimal or 'natural' state space. Following Kübler \& Polemarchakis (2004), such equilibria will be called Markov Equilibria (ME).

All results on the existence and properties of ME in Wang $(1993,1994)$ and the aforementioned and related papers are derived under the assumption that capital is the only asset available to consumers. This excludes the existence of a bubbly asset from the outset. However, as bubbly equilibria can potentially emerge in these economies without any change in fundamentals or the behavioral characteristics of agents, any characterization of the set of equilibria and their properties remains incomplete unless bubbly equilibria are also taken into account. Filling this gap is therefore the main contribution relative 
to Wang (1993) and similar studies of ME. Extending the terminology from Kübler \& Polemarchakis (2004), we call a ME which admits a bubble a Bubbly Markov Equilibrium (BME). Identifying conditions under which a BME exists and characterizing its properties is the general objective of this paper.

The first part of our analysis lays out a general method to construct potentially bubbly ME. This sets the stage to establish a general existence theorem for BME in the second part. A first major obstacle to construct ME in our setup is that the pointwise fixed point methods employed in Wang (1993) are no longer applicable. For this reason, our construction is based on monotone methods from functional analysis similar to Coleman (1991, 2000), or Greenwood \& Huffman (1995). This approach was successfully applied in Morand \& Reffett (2007) to study bubbleless ME, and we will show how it can be extended to study BME as well. The method to be developed is also constructive and can directly be employed to compute BME numerically in applications of our results.

The goal of the second part is to provide necessary and sufficient conditions under which the ME constructed is bubbly. Our main result shows that this is the case whenever the bubbleless equilibrium is Pareto inefficient. Such an inefficiency can be the result of dynamic inefficiency due to overaccumulation of capital as studied in Zilcha (1990) but may also be the result of inefficient intertemporal risk sharing between generations (see Barbie, Hagedorn \& Kaul (2007) for details). Thus, a major difference to the deterministic result in Tirole (1985) is that bubbles can emerge in stochastic economies which are dynamically efficient but suffer from inefficient risk sharing. While this result may sound intuitive, it requires new methods and proofs inherently different from the ones used in deterministic studies or stochastic exchange economies 1 Thus, our paper also offers a methodological contribution.

A major challenge to establish our existence result is that it requires a workable criterion to determine when an equilibrium allocation is Pareto inefficient. Building on the results from Chattopadhyay \& Gottardi (1999), a complete characterization of Pareto optimality in stochastic OLG production economies is provided in Barbie, Hagedorn \& Kaul (2007). The criterion employed in this paper essentially combines their results with the recursive formulations of Pareto optimality/suboptimality developed in Barbie \& Kaul (2015) who provide dominant root-type criteria necessary and sufficient for inefficiency in a stationary exchange economy with continuous state space. Similar criteria for efficiency/inefficiency are derived in Demange \& Laroque (2000). By exploiting certain monotonicity properties, our framework allows us to extend the results in Barbie \& Kaul (2015) and Demange \& Laroque (2000) and offer a complete characterization of inefficiency under a standard condition (Feller continuity) on the endogenous transition probability on the natural state space.

\footnotetext{
${ }^{1}$ Formally, this is because investments in capital and the bubbly asset being imperfect substitutes in our framework give rise to two Euler equations while there is only one such equation in the deterministic or pure exchange case.
} 
Based on this criterion, we establish our existence result by constructing a sequence of economies with a dividend-paying asset whose dividends converge to zerd2. Each such economy is known to have only efficient ME. Under some additional restrictions, the limiting ME of the benchmark economy is also efficient. Thus, a BME necessarily exists whenever the bubbleless equilibrium is known to be Pareto inefficient.

We expect our existence result to have many promising and interesting applications. One such application concerns the sustainability of government debt which is rolled over indefinitely between any two successive generations and is formally equivalent to a bubble. The sustainability of debt in the deterministic case was first analyzed in Diamond (1965). In the stochastic case, Ball, Elmendorf \& Mankiw (1998) argue that, roughly speaking, roll-over is possible when debt offers a riskless return lower than the growth rate of the economy. Our characterization which is based on the pricing kernel of the economy offers a clean theoretical foundation of this argument and permits to extend it to debt returns with a general stochastic structure. Further, our construction of BME provides an algorithm for explicitly determining sustainable debt policies and the optimal risk indexation of debt returns. These findings could also be valuable for applied studies, e.g., in the current discussion about sustainable debt levels, low interest rates and secular stagnation. Further potential applications, e.g., in the context of social security systems as studied in Gottardi \& Kübler (2011) are discussed below.

Bubbles can also emerge in models with heterogeneous, infinitely-lived households in the presence of borrowing constraints which are self-enforcing. A recent paper representative of this literature is Hellwig \& Lorenzoni (2009). They show that if borrowing constraints are positive and sustainable, they behave like a bubble in the sense that the resulting equilibrium allocation is the same as in an economy with unbacked public debt (see Theorem 2 in Hellwig \& Lorenzoni (2009)). Similar to the OLG-literature, bubbles in their model can only occur if equilibrium interest rates are sufficiently low (see Proposition 3 in Hellwig \& Lorenzoni (2009).)

A final large and growing branch of the literature studies the existence and properties of bubbly equilibria in the presence of financial frictions. Representatives of this literature are Farhi \& Tirole (2012), Martin \& Ventura (2012), and, more recently, Ikeda \& Phan (2016) or Miao, Wang \& Xu (2016). In the present paper, we choose not to include such frictions for at least three reasons. First, we would like to retain the deterministic case studied in Tirole (1985) as a natural reference point for our existence conditions. Second, as explained above, we will formulate our existence conditions in terms of the welfare properties of the bubbleless equilibrium based in criteria which are well-understood only in the frictionless case. Third, an important reason for introducing frictions in the first place was to explain the emergence of bubbles in dynamically efficient economies. Thus, an important contribution of our paper to the literature with frictions is that bubbles

\footnotetext{
${ }^{2}$ The idea of taking the limit of an economy with positive dividends to obatin bubbly equilibria was also used in Barbie \& Kaul (2015) and Aiyagari \& Peled (1991).
} 
can emerge in dynamically efficient economies even in the absence of such frictions.

The paper is organized as follows. Section 1 introduces the model and defines the concept of a Markov equilibrium. Section 2 develops a general approach to construct these equilibria. Section 3 contains the main results which state necessary and sufficient conditions under which the ME constructed is bubbly. Section 4 concludes; technical proofs and derivations are relegated to the Mathematical Appendices $\mathrm{A}$ and $\mathrm{B}$.

\section{The Model}

This section introduces the structure and assumptions of the basic model and formalizes the concept of a Markov equilibrium which will be at the core of the subsequent analysis.

\subsection{Production sector}

The production side is represented by a single firm which operates a linear homogeneous technology to produce an all-purpose output commodity using capital and labor as inputs. In addition, production in period $t$ is subjected to an exogenous random production shock $\theta_{t}>0$. At equilibrium, labor supply will be constant and normalized to unity. Given the shock, the intensive form production function $f: \mathbb{R}_{+} \longrightarrow \mathbb{R}_{+}$ determines production output $y_{t}$ in period $t$ from the existing stock of capital $k_{t} \geq 0$ as

$$
y_{t}=\theta_{t} f\left(k_{t}\right) .
$$

As in Wang (1993), shocks are i.i.d. over time with (marginal) distribution $\nu$ supported on the compact set $\Theta \subset \mathbb{R}_{++}$. Let $\theta_{\min }$ denote the minimal and $\theta_{\max }$ the maximal realization of the shock. The formal arguments in Section 3 assume that $\Theta$ is a finite set. The process $\left\{\theta_{t}\right\}_{t \geq 0}$ induces a probability space $(\Omega, \mathcal{F}, \mathbb{P})$ on which all random variables are defined and a filtration $\left\{\mathcal{F}_{t}\right\}_{t \geq 0}$ such that $\theta_{t}$ is $\mathcal{F}_{t}$-measurable. Throughout, the notion of an adapted stochastic process $\left\{\xi_{t}\right\}_{t \geq 0}$ refers to this filtration and implies that each $\xi_{t}$ can depend only on random variables $\theta_{n}, n \leq t$. Moreover, $\mathbb{E}_{t}[\cdot]:=\mathbb{E}\left[\cdot \mid \mathcal{F}_{t}\right]$ is the conditional expectations operator.

The following restrictions on $f$ are standard and will be imposed throughout the paper.

\section{Assumption 1}

The map $f: \mathbb{R}_{+} \longrightarrow \mathbb{R}_{+}$is $C^{2}$ with derivatives $f^{\prime \prime}<0<f^{\prime}$ and $\lim _{k \rightarrow 0} f^{\prime}(k)=\infty$. Moreover, there exists an upper bound $\bar{k}>0$ such that $\theta_{\max } f(k)<k$ whenever $k>\bar{k}$.

The previous restrictions ensure that $f$ is strictly increasing, strictly concave, and satisfies the so-called left-side Inada condition of an infinite capital return at zero. The 
final restriction excludes unbounded growth and ensures that any feasible allocation of capital, output, and consumption in our model is uniformly bounded.

Market clearing and profit maximizing behavior imply that the equilibrium wage $w_{t}$ and capital return $r_{t}$ are determined by the capital stock $k_{t}>0$ and the shock as $\theta_{t} \in \Theta$ as

$$
\begin{aligned}
w_{t} & =W\left(k_{t}, \theta_{t}\right):=\theta_{t}\left[f\left(k_{t}\right)-k_{t} f^{\prime}\left(k_{t}\right)\right] \\
r_{t} & =R\left(k_{t}, \theta_{t}\right):=\theta_{t} f^{\prime}\left(k_{t}\right) .
\end{aligned}
$$

Economically, equations (1a) and (1b) equate the prices of labor and capital to their marginal product in production which also implies that equilibrium profits are zero.

\subsection{Consumption sector}

The consumption sector consists of overlapping generations of consumers who live for two periods. For simplicity, there is no population growth and the size of each generation is normalized to unity. Young consumers earn income from supplying one unit of labor inelastically to the labor market while old consumers earn the proceeds on their investments made during the previous period.

To transfer income to the second period of life, there are two investment possibilities available to a young consumer in period $t$. First, she can invest in capital to earn the uncertain capital return $r_{t+1}$ in the next period per unit invested at time $t$. Second, she can invest in assets given by retradeable shares of a fruit tree (Lucas tree) which pay a constant non-random dividend $d \geq 0$ in each period. Let $p_{t} \geq 0$ denote the asset price per share in period $t \geq 0$. The total supply of shares is normalized to unity.

A young consumer in period $t$ observes her labor income $w_{t}>0$ and the buying price of shares $p_{t} \geq 0$ while taking the selling price $p_{t+1} \geq 0$ and the capital return $r_{t+1}>0$ as given random variables in her decision. The consumer chooses the desired investments in capital $s$ and in shares $z$ to maximize expected lifetime utility. Assuming an additive von-Neumann Morgenstern utility function $U\left(c^{y}, c^{o}\right)=u\left(c^{y}\right)+v\left(c^{o}\right)$ over lifetime consumption, the decision problem reads:

$$
\max _{z, s}\left\{u\left(w_{t}-z p_{t}-s\right)+\mathbb{E}_{t}\left[v\left(z\left(p_{t+1}+d\right)+s r_{t+1}\right)\right] \mid s \geq 0, z \geq 0, z p_{t}+s \leq w_{t}\right\} .
$$

Throughout, we impose the following standard restrictions on the utility functions.

\section{Assumption 2}

Both $g \in\{u, v\}$ are $C^{2}$ with derivatives satisfying $g^{\prime \prime}<0<g^{\prime}$ and $\lim _{c \rightarrow 0} g^{\prime}(c)=\infty$. Moreover, second period utility satisfies property $(U): c\left|v^{\prime \prime}(c)\right| \leq v^{\prime}(c)$ for all $\left.\left.c \in\right] 0, c_{\max }\right] 3$

\footnotetext{
${ }^{3}$ Here, $c_{\max }$ is a suitable upper bound for equilibrium consumption. It can formally be obtained if capital is restricted to the bounded state space defined below.
} 
The previous restrictions imply that utility functions in both periods are strictly increasing and concave and their boundary behavior excludes equilibrium allocations with zero consumption in any period of life. Furthermore, property (U) confines risk aversion of second period utility to the unit-interval which is again a standard although more restrictive condition also imposed in Magill \& Quinzii (2003), Morand \& Reffett (2007) or McGovern et al. (2013). In deterministic models such as Galor \& Ryder (1989), it ensures that savings are an increasing function of the expected capital return.

Capital investment $s_{t}$ in period $t$ determines the capital stock $k_{t+1}$ of the following period. Combining this with the first-order conditions of the decision problem (2), one obtains the following Euler equations which must hold in each period $t$ at equilibrium:

$$
\begin{aligned}
u^{\prime}\left(w_{t}-p_{t}-k_{t+1}\right) & =\mathbb{E}_{t}\left[r_{t+1} v^{\prime}\left(p_{t+1}+d+k_{t+1} r_{t+1}\right)\right] \\
u^{\prime}\left(w_{t}-p_{t}-k_{t+1}\right) p_{t} & =\mathbb{E}_{t}\left[\left(p_{t+1}+d\right) v^{\prime}\left(p_{t+1}+d+k_{t+1} r_{t+1}\right)\right] .
\end{aligned}
$$

Some results will require an additional joint restriction on the production technology $f$ and second-period utility $v$. To introduce this restriction, let $E_{\phi}(z):=\left|\frac{z \phi^{\prime}(z)}{\phi(z)}\right|, z \in \mathbb{R}$ the (absolute) elasticity of a differentiable function $\phi \neq 0$. Using this notation, define

$$
\begin{aligned}
& \left.\left.E_{v^{\prime}}^{\max }:=\sup \left\{E_{v^{\prime}}(c) \mid c \in\right] 0, c_{\max }\right]\right\} \\
& \left.\left.E_{v^{\prime}}^{\min }:=\inf \left\{E_{v^{\prime}}(c) \mid c \in\right] 0, c_{\max }\right]\right\} .
\end{aligned}
$$

The values in (44) define upper and lower bounds on risk aversion $E_{v^{\prime}}(c)=\frac{c\left|v^{\prime \prime}(c)\right|}{v^{\prime}(c)}$. The following assumption uses these values to obtain a lower bound on the elasticity of $f^{\prime}$.

\section{Assumption 3}

The production technology $f$ and second period utility $v$ satisfy the joint restriction

$$
E_{f^{\prime}}(k) \geq \frac{E_{v^{\prime}}^{\max }-E_{v^{\prime}}^{\min }}{1+E_{v^{\prime}}^{\max }-E_{v^{\prime}}^{\min }}
$$

for all $\left.k \in \mathbb{K}=] 0, k_{\max }\right]$ with $k_{\max }$ to be determined below and $E_{v^{\prime}}^{\max }$ and $E_{v^{\prime}}^{\min }$ by (4).

Unlike Assumptions 1 and 2, Assumption 3 is a non-standard restriction which will be needed for the monotone methods employed in this paper. Essentially, condition (5) imposes a uniform lower bound on the curvature of the function $f$ measured by $E_{f^{\prime}}$ which increases with the range of risk aversion $E_{v^{\prime}}^{\max }-E_{v^{\prime}}^{\min }$. It holds automatically if $v^{\prime}$ displays constant relative risk aversion in which case $E_{v^{\prime}}^{\max }=E_{v^{\prime}}^{\min }$, an assumption widely used in applied macroeconomic models. Further, since $0 \leq E_{v^{\prime}}^{\max }-E_{v^{\prime}}^{\min } \leq E_{v^{\prime}}^{\max } \leq 1$ due to Assumption 2, the r.h.s. in (5) is uniformly bounded from above by $\frac{1}{2}$. Thus, (5) holds for any production technology with $E_{f^{\prime}}$ uniformly bounded from below by $\frac{1}{2}$. An example is the Cobb-Douglas case $f(k)=k^{\alpha}$ with capital elasticity $0<\alpha \leq \frac{1}{2}$ which is a standard restriction imposed in virtually any empirical application. These observations 
reveal that Assumption 3 can be satisfied by restricting either the production or the consumer side of the economy. Also note that we do not impose an upper bound on $E_{f^{\prime}}$. Thus, we completely avoid the restrictive capital-income monotonicity condition $E_{f^{\prime}} \leq 1$ which is often imposed in the literature, cf. Wang (1993), Hauenschild (2002), and others. Finally, we remark that we could further relax Assumption 3 if some restrictions on the distribution $\nu$ were imposed. For our study in this paper, we decided not to impose such restrictions.

\subsection{Markov Equilibria (ME)}

The dividend payment $d \geq 0$ will be a key parameter in our analysis. For a given value $d \geq 0$, the economy is summarized by the list $\mathscr{E}_{d}=\langle u, v, f, \nu, d\rangle$ plus initial conditions for capital $k_{0}>0$ and the shock $\theta_{0} \in \Theta$. Specifically, we refer to the economy $\mathscr{E}:=\mathscr{E}_{0}$ in which dividend payments are zero as as the benchmark economy in our framework. Note that $\mathscr{E}$ essentially corresponds to the economy studied in Wang (1993).

The following definition is standard and provides the most general notion of equilibrium which reconciles market clearing and optimal behavior of all market participants with rational, self-confirming expectations of consumers.

\section{Definition 1}

Given initial values $k_{0}>0$ and $\theta_{0} \in \Theta$, a sequential equilibrium (SE) of $\mathscr{E}_{d}$ is an adapted stochastic process $\left\{w_{t}, r_{t}, p_{t}, k_{t+1}\right\}_{t \geq 0}$ which satisfies (1a,b) and (3a,b) for all $t \geq 0$.

Using the consumers' budget constraints, the equilibrium consumption processes are given by $c_{t}^{y}=w_{t}-p_{t}-k_{t+1}$ and $c_{t}^{o}=k_{t} r_{t}+p_{t}+d=\theta_{t} f\left(k_{t}\right)+d-c_{t}^{y}-k_{t+1}$ for all $t \geq 0$.

In this paper, we focus on a particular class of equilibria where all equilibrium variables are determined by time-invariant functions of some state variable $x_{t}$ which takes values in the state space $\mathbb{X}$. In the literature, such equilibria are called Recursive Equilibria $(R E)$. We confine ourselves to a particular class of recursive equilibria where the state variable is $x_{t}=\left(k_{t}, \theta_{t}\right)$. The underlying state space $\mathbb{X}$ is called the natural state space. Note that the factor price mappings $W$ and $R$ from (1a,b) already satisfy this property. Following the terminology of Kübler \& Polemarchakis (2004), RE on the natural state space are called Markov Equilibria (ME). In the following definition, $\mathbb{X} \subset \mathbb{R}_{++} \times \Theta$ is assumed to be a non-empty Borel set which will be constructed explicitly in the next section.

\section{Definition 2}

A SE of $\mathscr{E}_{d}$ is called a Markov equilibrium (ME) on $\mathbb{X}$ if there exists measurable mappings $K_{d}^{E}: \mathbb{X} \longrightarrow \mathbb{R}_{++}$and $P_{d}^{E}: \mathbb{X} \longrightarrow \mathbb{R}_{+}$such that $k_{t+1}=K_{d}^{E}\left(k_{t}, \theta_{t}\right)$ and $p_{t}=P_{d}^{E}\left(k_{t}, \theta_{t}\right)$ for all $t \geq 0$ and all $x_{0}=\left(k_{0}, \theta_{0}\right) \in \mathbb{X}$. 
A primary goal of this paper is to study $\mathrm{ME}\left(K^{E}, P^{E}\right)$ of the benchmark economy $\mathscr{E}=\mathscr{E}_{0}$ where dividend payments are zero (we will occasionally drop the subscript if $d=0$ ). In particular, we ask whether such equilibria admit a bubble, i.e., can be supported by a non-zero asset price process. Extending the previous terminology, we refer to a ME which admits a bubble as a Bubbly Markov Equilibrium (BME). Formally, we have

\section{Definition 3}

A ME $\left(K^{E}, P^{E}\right)$ of $\mathscr{E}$ is called bubbly if $P^{E} \neq 0$ and bubbleless if $P^{E}=0$.

Below we show that any BME satisfies $P^{E}>0$, i.e., the price of the bubbly asset is everywhere positive. Note that by adding the Euler equations (3a,b), one could also reinterpret the bubbly asset as a bubble on capital, i.e., a deviation from its fundamental value rather than being a separate asset. Intuitively, one can imagine that the firm issues a combined asset which subsumes both investment in capital and the bubble. In this way, we can attach a bubble to any existing asset by adding its price to the fundamental price of the asset.

In addition to their theoretical appeal, bubbly ME have several important applications and admit various alternative interpretations. One such application concerns the sustainability and optimal risk structure of governmental debt. Suppose in each period $t$, a government issues one-period bonds with unit price and (risk-indexed) return $r_{t+1}^{*}$ to finance its current debt $b_{t}>0$. Then, the process $\left\{b_{t}\right\}_{t \geq 0}$ which evolves as $b_{t+1}=r_{t+1}^{*} b_{t}$ is formally equivalent to a bubble in our previous framework. Exploiting this equivalence, the value $P^{E}\left(x_{t}\right)$ defines the maximum level of debt that is sustainable if the current fundamental state is $x_{t} \in \mathbb{X}$. Further, the optimal risk structure of the return offered in period $t$ needed to sustain this maximum level is determined by the random variable

$$
r_{t+1}^{*}:=R^{*}\left(x_{t}, \cdot\right)=\frac{P^{E}\left(K^{E}\left(x_{t}\right), \cdot\right)}{P^{E}\left(x_{t}\right)} .
$$

The existence of a BME is therefore equivalent to a positive equilibrium level of debt that can be sustained without further stabilization such as taxation, etc. Also note that (6) would permit to explicitly compute the Arrow-Debreu prices of risk at equilibrium.

An alternative interpretation of a BME is that of a monetary equilibrium in which a fixed quantity $M>0$ of fiat money is exchanged between successive generations. In this case, the price $p_{t}>0$ corresponds to real money balances in period $t$.

One can also interpret a BME as an equilibrium with a social security system in which $p_{t}>0$ represents the transfers from young to old consumers in period $t \geq 0$. A particular appealing feature that follows from the Euler equation (3b) is that such a system is time consistent in the sense that no generation has an incentive to change it (see Hillebrand (2011) for an application of this concept). Thus, a BME directly implies the existence of a time-consistent Social Security system. 
In the following section we show that the properties of the (unique) bubbleless ME of $\mathscr{E}$ are key to construct the state space $\mathbb{X}$ associated with any ME of $\mathscr{E}_{d}$ where $d \geq 0$.

\subsection{Restricting the state space}

It is shown in Hillebrand (2014) that property (U) from Assumption 1 is already sufficient for the benchmark economy $\mathscr{E}$ to possess a unique bubbleless ME. In this case, the equilibrium mappings are given by $P_{0}^{E} \equiv 0$ and $K_{0}^{E}=K_{0} \circ W$ where $K_{0}: \mathbb{R}_{++} \longrightarrow \mathbb{R}_{++}$ determines the unique solution $k=K_{0}(w)$ to the implicit condition

$$
G_{0}(k, w):=u^{\prime}(w-k)-\mathbb{E}_{\nu}\left[R(k, \cdot) v^{\prime}(k R(k, \cdot))\right]=0 .
$$

Note that the implicit function theorem implies that $K_{0}$ is $C^{1}$, strictly increasing, and $0<K_{0}(w)<w$ for all $w>0$. The capital process along the bubbleless ME evolves as

$$
k_{t+1}=K_{0}^{E}\left(k_{t}, \theta_{t}\right)=K_{0} \circ W\left(k_{t}, \theta_{t}\right) \text {. }
$$

Equation (8) is precisely the representation of equilibrium studied in Wang (1993). To rule out degenerate cases in which capital converges to zero with positive probability, he imposes the additional restriction $\lim _{k \searrow_{0}} \partial_{k} K_{0}^{E}\left(k, \theta_{\text {min }}\right)>1$, cf. Theorem 4.3 in Wang (1993). It ensures existence of a lower bound $\underline{k}>0$ such that $K^{E}(k, \theta)>k$ for all $\theta \in \Theta$ whenever $k \leq \underline{k}$. For most of the following analysis, however, it suffices to work with a weaker condition which only excludes that capital converges to zero with probability one. Only later will the stronger requirement of Wang (1993) be necessary.

Before introducing such restrictions formally, a crucial observation is that the bubbleless ME is fully described by the map $K_{0}$ defined on the one-dimensional set $\mathbb{W} \subset \mathbb{R}_{++}$of equilibrium wages. We will show in the next section that the same structure obtains in the bubbly case and also along any ME of $\mathscr{E}_{d}, d>0$. In each case, the equilibrium mappings in Definition 2 take the form $P_{d}^{E}=P_{d} \circ W$ and $K_{d}^{E}=K_{d} \circ W$ with $P_{d}$ and $K_{d}$ defined on $\mathbb{W}$. Thus, any ME is completely described by mappings defined on a one-dimensional set $\mathbb{W}$ which we will refer to as the reduced state space. For this reason, the pair $\left(P_{d}, K_{d}\right)$ will also be referred to as a ME of $\mathscr{E}_{d}$.

It will be convenient to impose restrictions on the reduced state space $\mathbb{W}$ rather than $\mathbb{X}$ directly. For this reason, we state the aforementioned boundary properties in terms of the wage process along the bubbleless equilibrium which evolves as

$$
w_{t+1}=W_{0}^{E}\left(w_{t}, \theta_{t+1}\right):=W\left(K_{0}\left(w_{t}\right), \theta_{t+1}\right) .
$$

Mathematically, this representation of the equilibrium dynamics is equivalent to (8). The following assumption rules out that the wage process (91) converges to zero with probability one. 


\section{Assumption 4}

The map $W_{0}^{E}$ defined in (9) satisfies $\liminf _{w \searrow_{0}} W_{0}^{E}\left(w, \theta_{\max }\right) / w>1$.

Assumption 4 does not seem too restrictive, as it essentially excludes only economies for which capital converges to zero with probability one. In our view, such economies are not very interesting for the primary objective of this paper to study bubbly equilibria.

Assumptions 1 and 4 together with continuity of $W_{0}^{E}$ ensure that the set of fixed points of $W_{0}^{E}\left(\cdot, \theta_{\max }\right)$ is non-empty and compact. Thus, defining

$$
w_{\max }:=\min \left\{w>0 \mid w=W_{0}^{E}\left(w, \theta_{\max }\right)\right\}
$$

allows us to use $\left.\mathbb{W}:=] 0, w_{\max }\right]$ as the reduced state space. Note that $\mathbb{W}$ is self-supporting in the sense that $w \in \mathbb{W}$ implies $W_{0}^{E}(w, \theta) \in \mathbb{W}$ for all $\theta \in \Theta$. Further, $W_{0}^{E}\left(\cdot, \theta_{\max }\right)$ has $w_{\max }$ as its unique fixed point which is globally asymptotically stable on $\mathbb{W} \mathbb{4}$

Setting $k_{\max }:=K_{0}\left(w_{\max }\right)$ and $\left.\left.\mathbb{K}=\right] 0, k_{\max }\right]$ permits to define $\mathbb{X}:=\mathbb{K} \times \Theta$ as the natural state space from Definition 2 along the bubbleless ME. In the next section, we show that these choices for $\mathbb{W}$ and $\mathbb{X}$ extend to the bubbly case and any ME of $\mathscr{E}_{d}$ if $d>0$. Thus, a major advantage of Assumption 4 is that it permits a bounded state space.

Some results of Section 3 will even require that $\mathbb{W}$ and $\mathbb{X}$ can be chosen compact along the bubbleless ME. In such cases, the following stronger restriction is imposed which rules out that the wage process (9) converges to zero even with positive probability.

\section{Assumption 5}

There exists $\underline{w}>0$ such that $W_{0}^{E}\left(w, \theta_{\min }\right)>w$ for all $w \leq \underline{w}$ with $W_{0}^{E}$ defined in (9)).

Assumption 5 ensures that $W_{0}^{E}\left(\cdot, \theta_{\min }\right)$ has at least one positive fixed point. Defining

$$
w_{\min }:=\min \left\{w>0 \mid w=W_{0}^{E}\left(w, \theta_{\min }\right)\right\}
$$

ensures that $\overline{\mathbb{W}}:=\left[w_{\min }, w_{\max }\right]$ is a compact self-supporting set for the dynamics (9). While this would also permit to choose the state space $\mathbb{X}$ compact along the bubbleless ME, these choices neither extend to the bubbly case nor to a ME of $\mathscr{E}_{d}$ if $d>0$.

A direct implication of Assumption 5 is $\liminf _{w \searrow 0} W_{0}^{E}\left(w, \theta_{\min }\right) / w \geq 1$, which is mathematically essentially equivalent to (although slightly weaker than) the condition in Wang (1993) discussed above. Further, since shocks in (9) are multiplicative, Assumption 5 implies Assumption 4 whenever $\theta_{\max }>\theta_{\min }$.

A potential problem with both Assumptions 4 and 5 is that they refer to derived objects and are not stated in terms of the primitives of the model. For this reason, we present two additional results which state restrictions on the primitives under which the stronger

\footnotetext{
${ }^{4}$ This uniqueness property will be important to obtain several results including Theorem 1 Otherwise, we could have defined $w_{\max }$ in (10) to be the maximum fixed point of $W_{0}^{E}\left(\cdot, \theta_{\max }\right)$.
} 
Assumption 5 holds. These restrictions should also be easy to verify in any numerical or empirical application of the model. As they are sufficient but not necessary, we will continue to work with Assumptions 4 and 5 in the following analysis.

\section{Lemma 1.1}

In addition to Assumptions 1 and 2, let the following conditions hold:

$$
\text { (a) } \liminf _{k \searrow 0} \frac{W\left(k, \theta_{\text {min }}\right)}{k}>1 \quad \text { (b) } \liminf _{k \searrow 0} \frac{\mathbb{E}_{\nu}\left[R(k, \cdot) v^{\prime}(k R(k, \cdot))\right]}{u^{\prime}\left(W\left(k, \theta_{\min }\right)-k\right)}>1 .
$$

Then, Assumption 5 is satisfied.

Condition (a) is necessary for Assumption 5, as it excludes a poverty trap in which capital would converge to zero under the minimal shock $\theta_{\text {min }}$, independently of any restriction on preferences. A sufficient restriction under which (a) holds is the so-called 'strengthened Inada' (SI) condition $\lim _{k \searrow_{0}} k f^{\prime \prime}(k)=\infty$ introduced in Galor \& Ryder (1989). Note that this condition is implied by the left-side Inada condition from Assumption 1 whenever $E_{f^{\prime}}$ is bounded away from zero. Thus, it holds automatically under our Assumption 3 imposed below whenever there is some variation in risk aversion of second-period utility. As $\lim _{k \searrow 0} f^{\prime}(k)=\infty$, condition (b) requires that $\mathbb{E}_{\nu}\left[\frac{\operatorname{id}_{\Theta}(\cdot) v^{\prime}(k R(k, \cdot))}{u^{\prime}\left(W\left(k, \theta_{\min }\right)-k\right)}\right]$ is either bounded below or at least does not converge to zero 'too fast' as capital converges to zero (here, $\operatorname{id}_{\Theta}(\cdot)$ is the identity map on $\left.\Theta\right)$. As $\theta \mapsto \theta v^{\prime}\left(\theta f^{\prime}(k) k\right)$ is non-decreasing due to $E_{v^{\prime}} \leq 1$, a sufficient condition for (b) is therefore

$$
\liminf _{k \searrow 0} \frac{v^{\prime}\left(k R\left(k, \theta_{\min }\right)\right)}{u^{\prime}\left(W\left(k, \theta_{\min }\right)-k\right)}>0
$$

which is similar to the existence conditions in Li \& Lin (2012) for the deterministic case. An alternative restriction to enforce (a) is the so-called 'non-vanishing labor share' (NLS) condition $\lim \inf _{k \searrow 0} \frac{f(k)}{k f^{\prime}(k)}>1$ which is generally stronger and implies SI whenever $f(0)=0.5$ The NLS condition is again satisfied if $E_{f^{\prime}}$ is bounded away from zero. Importantly, in the special case often studied in the literature where $v(c)=\beta u(c)$ for some discount factor $\beta>0$, NLS combined with Assumptions 1 and 2 also implies (12) and, therefore, condition (b). The same is true if instead $f(0)>0$ which holds, e.g., with CES production and an elasticity of substitution larger than one. As we did not find these last results in the literature, we state them in the following lemma 6

\section{Lemma 1.2}

Under Assumptions 1 and 2, suppose $\liminf _{k \searrow 0} \frac{f(k)}{k f^{\prime}(k)}>1$. Then condition (a) holds. Moreover, if either $v(c)=\beta u(c)$ for all $\left.c \in] 0, c_{\max }\right]$ or $f(0)>0$, then $(b)$ is also satisfied.

\footnotetext{
${ }^{5}$ To see this, suppose $f(0)=0$. Then, by L'Hopital's rule, $\lim _{k \searrow 0} \frac{f(k)}{k f^{\prime}(k)}=\lim _{k \searrow 0} \frac{1}{1-E_{f^{\prime}}(k)}$. The condition thus requires $\lim _{k \searrow_{0}} E_{f^{\prime}}(k)>0$. As $\lim _{k \searrow_{0}} f^{\prime}(k)=\infty$, this can only hold under SI.

${ }^{6}$ For the deterministic OLG growth model, Konishi \& Perera-Tallo (1997) establish existence of a non-trivial steady state equilibrium when NLS holds and lifetime utility is homothetic, see their Corollary 1 on page 535. These restrictions are somewhat similar to those of Lemma 1.2 for the present stochastic case.
} 


\section{Constructing Markov Equilibria}

The pointwise construction of ME employed in Wang (1993) and the previous section is available only in the bubbleless case. For this reason, the following sections develop a more general approach which is based on methods from functional analysis similar to Morand \& Reffett (2007). It is shown in Hillebrand (2014) that this approach is equivalent to the pointwise construction in Wang (1993) in the bubbleless case. Our method permits to construct ME of the general class of economies $\mathscr{E}_{d}, d \geq 0$ introduced in the previous section. Identifying conditions under which the solution obtained for $d=0$ defines a bubbly ME of the benchmark economy $\mathscr{E}$ then becomes a separate issue to be explored in Section 3 .

The following sections throughout impose Assumptions 1, 2, and 4, Using the results from Section 1.4, define $w_{\max }$ as in (10) and the reduced state space $\left.\left.\mathbb{W}=\right] 0, w_{\max }\right]$, $k_{\max }:=K_{0}\left(w_{\max }\right)$ by $\left.\left.(7), \mathbb{K}=\right] 0, k_{\max }\right]$, and the natural state space $\mathbb{X}=\mathbb{K} \times \Theta$.

\subsection{Defining an operator $T_{d}$}

Given $d \geq 0$, the following analysis aims to construct ME of $\mathscr{E}_{d}$ as fixed points of an operator $T_{d}$ defined on some suitably chosen function space $\mathscr{G}$. To restrict the class of candidate equilibrium functions $\mathscr{G}$, a first and crucial observation is that the current state $x_{t}=\left(k_{t}, \theta_{t}\right)$ enters the Euler equations $(3 \mathrm{a}, \mathrm{b})$ only through the wage $\left.w_{t}=W\left(x_{t}\right)\right]$ Therefore, we conjecture that, similar to the bubbleless equilibrium, the mappings from Definition 2 can be written as $K_{d}^{E}=K_{d} \circ W$ and $P_{d}^{E}=P_{d} \circ W$ where $K_{d}: \mathbb{W} \longrightarrow \mathbb{K}$ and $P_{d}: \mathbb{W} \longrightarrow \mathbb{R}_{+}$. Under this hypothesis, the problem of determining a ME is equivalent to determining the two functions $\left(K_{d}, P_{d}\right)$ consistent with the Euler equations (3a,, $\left.\mathrm{b}\right)$. Moreover, we will show below that any solution $P_{d}$ uniquely determines the associated capital function $K_{d}$. Thus, we are essentially left to determine the function $P_{d}$. We restrict our search for this solution to the function space

$$
\mathscr{G}:=\left\{\begin{array}{l|l}
P: \mathbb{W} \longrightarrow \mathbb{R}_{+} & \begin{array}{l}
P \text { is continuous } \\
w \mapsto P(w) \text { is weakly increasing } \\
w \mapsto w-P(w) \text { is weakly increasing }
\end{array}
\end{array}\right\} \text {. }
$$

The space $\mathscr{G}$ is endowed with the usual pointwise ordering, i.e., $P_{1} \geq P_{2}\left(P_{1}>P_{2}\right)$ iff $P_{1}(w) \geq P_{2}(w)\left(P_{1}(w)>P_{2}(w)\right)$ for all $w \in \mathbb{W}$.

The previous insights greatly simplify the construction of ME because they permit to reduce the problem of determining two functions $\left(P_{d}^{E}, K_{d}^{E}\right)$ both defined on $\mathbb{X}$ to

\footnotetext{
${ }^{7}$ This property rests crucially on the i.i.d. structure of the shock process. While this will simplify the subsequent construction of ME considerably, we expect the underlying principle along with most of the results to carry over to more general classes of economies including correlated production shocks. Clearly, in this case the function space $\mathscr{G}$ consists of mappings defined on $\mathbb{X}$ rather than $\mathbb{W}$.
} 
finding a single function $P_{d}$ defined on the one-dimensional space $\mathbb{W}$. In the sequel we will construct $P_{d}$ as a fixed point of some operator $T_{d}$ defined on $\mathscr{G}$. The additional monotonicity restrictions in (13) will be necessary for this operator to be well-defined.

Let $d \geq 0$ be arbitrary but fixed. The key ingredient to construct the operator $T_{d}$ are the Euler equations (3a,b). The idea is as follows: At some fixed point in time, suppose next period's asset price is determined by some function $P \in \mathscr{G}$ of next period's wage. Then, for any current state $w \in \mathbb{W}$, the current asset price $p$ and capital investment $k$ must solve the Euler equations (3a, b). Given $P \in \mathscr{G}$ and some fixed $w \in \mathbb{W}$, let

$$
\begin{aligned}
H^{1}(k, p ; w, P, d):= & u^{\prime}(w-p-k) \\
& -\mathbb{E}_{\nu}\left[R(k, \cdot) v^{\prime}(P(W(k, \cdot))+d+k R(k, \cdot))\right] \\
H^{2}(k, p ; w, P, d):= & u^{\prime}(w-p-k) p \\
& -\mathbb{E}_{\nu}\left[(P(W(k, \cdot))+d) v^{\prime}(P(W(k, \cdot))+d+k R(k, \cdot))\right]
\end{aligned}
$$

which are defined for all $0<k<k_{\max }$ and $p \geq 0$ such that $k+p<w$. Then, for any fixed $\tilde{w} \in \mathbb{W}$, the problem is to determine $\tilde{k} \in \mathbb{K}$, and $\tilde{p} \geq 0$ such that $\tilde{k}+\tilde{p}<\tilde{w}$ and

$$
H^{1}(\tilde{k}, \tilde{p} ; \tilde{w}, P, d)=H^{2}(\tilde{k}, \tilde{p} ; \tilde{w}, P, d)=0 .
$$

First, consider the problem (15) for $d=0$. For this case, we have the following result.

\section{Lemma 2.1}

Let Assumptions 1, 2, and 4 be satisfied and suppose $d=0$. Then, for any $P \in \mathscr{G}$ and $\tilde{w} \in \mathbb{W}$, there is a unique solution $\tilde{p} \geq 0$ and $\tilde{k} \in \mathbb{K}$ to (15).

Lemma 2.1 permits to define functions $T P: \mathbb{W} \longrightarrow \mathbb{R}_{+}$and $K_{P}: \mathbb{W} \longrightarrow \mathbb{R}_{++}$which determine the unique solution to (15) if $d=0$, i.e., $T P(\tilde{w}):=\tilde{p}$ and $K_{P}(\tilde{w}):=\tilde{k}$ for each $\tilde{w} \in \mathbb{W}$. This induces an operator $T$ on $\mathscr{G}$ which associates with any function $P \in \mathscr{G}$ the new function $T(P):=T P$. We also denote by $K_{\bullet}$ the operator on $\mathscr{G}$ which assigns to $P \in \mathscr{G}$ the function $K_{P} 8$

The following result shows that $T$ maps $\mathscr{G}$ into itself and establishes several additional properties. Here, the additional restriction (15) from Assumption 3 is needed to ensure that $T$ maps monotonic functions to monotonic functions.

\section{Lemma 2.2}

In addition to Assumptions 1, 2, and 4, let Assumption 3 hold. Then $T: \mathscr{G} \longrightarrow \mathscr{G}$. Further, for each $P \in \mathscr{G}$ the following holds:

(i) $T(P)<\mathrm{id}_{\mathbb{W}}, P>0$ implies $T(P)>0$ while $P=0$ implies $T(P)=0$.

(ii) $K_{P}$ is continuous and increasing, $K_{P} \leq K_{0}<\operatorname{id}_{\mathbb{W}}$ and $P>0$ implies $K_{P}<K_{0}$.

\footnotetext{
${ }^{8}$ As $K_{P}$ yields the solution $K_{0}$ defined by (7) for $P \equiv 0$, this notation is consistent with Section 1.4 .
} 
In a second step, consider now the problem (15) for $d>0$. Observe that this problem is identical to the case where $d=0$ if $P$ is replaced by the function $\hat{P}=P+d$, i.e., $\hat{P}(w):=P(w)+d$ for all $w \in \mathbb{W}$. Clearly, $P \in \mathscr{G}$ implies $P+d \in \mathscr{G}$ for all $d \geq 0$. Thus, define for each fixed $d \geq 0$ the operator $T_{d}$ on $\mathscr{G}$ as

$$
T_{d}(P)=T(P+d)
$$

Then, by Lemmata 2.1 and 2.2 , for each $P \in \mathscr{G}, \tilde{w} \in \mathbb{W}$ and fixed $d \geq 0$, the unique solution to (15) is given by $\tilde{p}=T_{d} P(\tilde{w})$ and $\tilde{k}=K_{P+d}(\tilde{w})$. In particular, $T_{0}=T$. The relation (16) shows that $T_{d}$ inherits all properties derived above for $T$. In particular, $T_{d}$ maps $\mathscr{G}$ into itself and $T_{d}(P)<\mathrm{id} \mathbb{W}$ for all $P \in \mathscr{G}$.

\subsection{Montonicity properties of $T_{d}$}

We conjecture - and prove in the next subsection - that a fixed point of $T_{d}$, i.e., a function $P_{d}^{*} \in \mathscr{G}$ such that $P_{d}^{*}=T_{d} P_{d}^{*}$ together with the induced capital function $K_{d}^{*}=K_{P_{d}^{*}+d}$ define a ME of $\mathscr{E}_{d}$. In this regard, the last result from Lemma 2.2 implies $K_{d}^{*} \leq K_{0}$ with the latter defined by (7). This property permits to employ $\left.\left.\mathbb{W}=\right] 0, w_{\max }\right]$ as the reduced state space and $\mathbb{X}=\mathbb{K} \times \Theta$ as the natural state space along any ME.

Our ultimate goal in this paper is to prove the existence of a BME which corresponds to a non-trivial fixed point $P_{0}^{*}>0$ of $T$. Unfortunately, however, Lemma 2.2 already showed that the trivial solution $P=0$ is always a fixed point of $T$, so a mere existence result will not help. Instead, we will explicitly construct fixed points as pointwise limits of sequences of function. The method is similar to the one developed in Greenwood \& Huffman (1995), see also Morand \& Reffett (2003, 2007).

A key property for this construction to be successful is that $T_{d}$ be monotonic which, by (16) is equivalent to monotonicity of $T$ which we will consider first. A major obstacle to establish this property globally on $\mathscr{G}$ is that the methods from differential calculus including the implicit function theorem are not available for all functions in $\mathscr{G}$. To remedy this problem, we will temporarily restrict ourselves (respectively $T$ ) to the smaller set

$$
\mathscr{G}^{\prime}:=\left\{P \in \mathscr{G} \mid P \text { is } C^{1}\right\}
$$

of continuously differentiable functions in $\mathscr{G}$. Observe that $\mathscr{G}^{\prime}$ still contains the trivial solution $P \equiv 0$. The next result shows that $T$ maps $\mathscr{G}^{\prime}$ into itself.

\section{Lemma 2.3}

Under the hypotheses of Lemma 2.2, $P \in \mathscr{G}^{\prime}$ implies $T P \in \mathscr{G}^{\prime}$.

The following result now establishes the monotonicity of $T$ on $\mathscr{G}^{\prime}$ which will turn out to be sufficient to apply the construction principle below. In addition, we show that $K_{\bullet}$ is strictly decreasing on $\mathscr{G}^{\prime}$ which resembles the usual crowding-out effect of assets. 


\section{Lemma 2.4}

Let Assumptions 1, 2, and 4 hold. Then, $T$ is monotonically increasing on $\mathscr{G}^{\prime}$, i.e., for any $P_{0}, P_{1} \in \mathscr{G}^{\prime}, P_{1} \geq P_{0}$ implies $T\left(P_{1}\right) \geq T\left(P_{0}\right)$ and $P_{1}>P_{0}$ implies $T\left(P_{1}\right)>T\left(P_{0}\right)$. Moreover, $K_{\bullet}$ is monotonically decreasing on $\mathscr{G}^{\prime}$.

It follows directly from (16) that the operator $T_{d}$ inherits again all previous properties from $T$. In particular, $T_{d}$ is monotonic on $\mathscr{G}^{\prime}$ and maps this subclass into itself. In addition, the map $d \mapsto T_{d}$ is monotonic in the sense that $d_{1} \geq d_{0}$ implies $T_{d_{1}} P \geq T_{d_{0}} P$ for all $P \in \mathscr{G}^{\prime}$. For later reference, we state these properties formally in the next result.

\section{Corollary 2.1}

Under the hypotheses of Lemma 2.4, $T_{d}$ satisfies the following monotonicity properties:

(i) For all $d \in \mathbb{R}_{+}$and $P_{0}, P_{1} \in \mathscr{G}^{\prime}: P_{1} \geq(>) P_{0}$ implies $T_{d} P_{1} \geq(>) T_{d} P_{0}$.

(ii) For all $P \in \mathscr{G}^{\prime}$ and $d_{0}, d_{1} \in \mathbb{R}_{+}: d_{1} \geq(>) d_{0}$ implies $T_{d_{1}} P \geq(>) T_{d_{0}} P$.

\subsection{Constructing $\mathrm{ME}$ as fixed points of $T_{d}$}

Let $d \geq 0$ be arbitrary but fixed. We are now in a position to construct ME of $\mathscr{E}_{d}$ as fixed points of $T_{d}$. For $m \in \mathbb{N}$, let $T_{d}^{m}$ denote the $m$-fold composition of $T_{d}$ with itself,

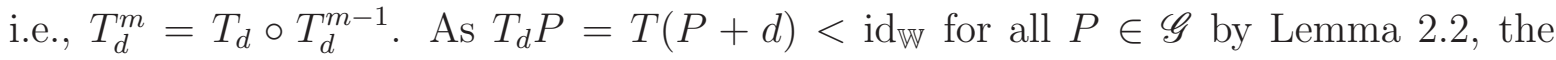
identity map $\operatorname{id}_{\mathbb{W}} \in \mathscr{G}^{\prime}$ defines a natural upper bound for any fixed point of $T_{d}$. Thus, define the sequence of functions $\left(P_{d}^{m}\right)_{m \geq 0}$ recursively by setting $P_{d}^{0} \equiv P_{0}:=\mathrm{id}_{\mathbb{W}}$ and $P_{d}^{m}:=T_{d}\left(P_{d}^{m-1}\right)=T_{d}^{m} P_{0}$. By Lemma 2.3, this sequence is well-defined and $P_{d}^{m} \in \mathscr{G}^{\prime}$ for all $m \geq 0$. Further, $P_{d}^{1}<P_{d}^{0}$ implies $P_{d}^{m+1}<P_{d}^{m}$ for all $m \geq 0$ by monotonicity of $T_{d}$, i.e., $\left(P_{d}^{m}\right)_{m \geq 0}$ is strictly decreasing. Thus, the pointwise limit

$$
P_{d}^{*}(w):=\lim _{m \rightarrow \infty} P_{d}^{m}(w)=\lim _{m \rightarrow \infty} T_{d}^{m} P_{0}(w)
$$

is well-defined for all $w \in \mathbb{W}$ as $\left(P_{d}^{m}(w)\right)_{m \geq 0}$ is a strictly decreasing sequence of real numbers bounded by zero. We show that the limiting function satisfies $P_{d}^{*} \in \mathscr{G}$. For each $m \geq 1, P_{d}^{m} \in \mathscr{G}$ implies that $w \mapsto P_{d}^{m}(w)$ and $w \mapsto w-P_{d}^{m}(w), w \in \mathbb{W}$ are both increasing. Thus, for any $0<w_{1}<w_{2} \leq w_{\max }$ the inequalities $P_{d}^{m}\left(w_{1}\right) \leq P_{d}^{m}\left(w_{2}\right)$ and $w_{1}-P_{d}^{m}\left(w_{1}\right) \leq w_{2}-P_{d}^{m}\left(w_{2}\right)$ being true for all $m \geq 1$ also hold in the limit and imply that $P_{d}^{*}$ inherits the previous monotonicity properties. Using an argument developed and proved in Morand \& Reffett (2003, p.1369), these properties already imply continuity of $P_{d}^{*}$. Thus, $P_{d}^{*} \in \mathscr{G}$. Note, however, that we can not be certain that $P_{d}^{*} \in \mathscr{G}^{\prime}$.

The previous findings lead to the following main result.

\section{Theorem 1}

Let Assumptions 1, 2, 3, and 4 hold. Then, for each $d \geq 0$ the functions $P_{d}^{*}$ defined in (18) and $K_{d}^{*}:=K_{P_{d}^{*}+d}$ satisfy the following: 
(i) $P_{d}^{*}$ is a fixed point of $T_{d}$ which satisfies $P_{d}^{*}>0$ for $d>0$ and either $P_{0}^{*}>0$ or $P_{0}^{*}=0$ if $d=0$. Moreover, $d>d^{\prime} \geq 0$ implies $P_{d}^{*} \geq P_{d^{\prime}}^{*}$ and $K_{d}^{*}<K_{d^{\prime}}^{*}$.

(ii) Both mappings $P_{d}^{*}$ and $K_{d}^{*}$ are continuous and increasing.

(iii) $K_{d}^{E}:=K_{d}^{*} \circ W$ and $P_{d}^{E}:=P_{d}^{*} \circ W$ is a $M E$ of $\mathscr{E}_{d}$ on $\mathbb{X}=\mathbb{K} \times \Theta$.

Theorem 1 shows that our algorithm delivers a unique ME for each $d \geq 0$ with asset prices being strictly positive whenever $d>0$. Moreover, (i) shows that these prices increase with dividend payments, which is certainly what one would expect. Further, higher asset prices and dividends reduce capital formation, which is the usual crowding out effect. Intuitively, consumers form less capital when asset pay-offs in the second period are higher. Assertion (ii) shows that both capital and asset prices increase with first-period income, i.e., higher income increases investment in both capital and assets.

For $d=0$, the previous construction also delivers a unique $\mathrm{ME}\left(K_{0}^{*}, P_{0}^{*}\right)$ of $\mathscr{E}$. However, it may be the case that $P_{0}^{*}=0$. Clearly, this would imply $K_{0}^{*}=K_{0}$ defined by (7) which yields precisely the bubbleless equilibrium studied in Section 1.4. Therefore, the main question to be answered in the remainder of the paper is, when does $P_{0}^{*}>0$ hold? Before exploring this question in the next section, we present an alternative way to construct the ME from Theorem 1 for the benchmark economy $\mathscr{E}$. The proof of our main existence result will be based on this construction. The idea is to obtain the ME of $\mathscr{E}$ as the limit of ME of dividend economies $\mathscr{E}_{d}$ as $d$ goes to zero. Formally, let $\left(d_{n}\right)_{n \geq 1}$ be a decreasing sequence of dividends such that $d_{n} \geq 0$ for all $n$ and $\lim _{n \rightarrow \infty} d_{n}=0$. By Theorem 1, for each $n \geq 1$ the functions $P_{d_{n}}^{*}$ defined by (18) and $K_{d_{n}}^{*}=K_{P_{d_{n}}^{*}+d_{n}}$ define a ME of $\mathscr{E}_{d_{n}}$. The following result shows that the sequence of ME constructed in this fashion indeed converges (pointwise) to the ME of $\mathscr{E}$ defined by Theorem 1 .

\section{Lemma 2.5}

For any positive dividend sequence $\left(d_{n}\right)_{n \geq 1}$ converging monotonically to zero, the induced sequence of $\mathrm{ME}\left(K_{d_{n}}^{*}, P_{d_{n}}^{*}\right)_{n \geq 1}$ from Theorem 1 converges pointwise to $\left(K_{0}^{*}, P_{0}^{*}\right)$.

\section{Existence of Bubbly Markov Equilibria}

In this section we establish necessary and sufficient conditions under which the ME $\left(K_{0}^{*}, P_{0}^{*}\right)$ constructed in Theorem 1 is bubbly, i.e., $P_{0}^{*}>0$. Our main result stated as Theorem 2 below shows that this is the case whenever the bubbleless equilibrium derived in Section 1.4 is Pareto inefficient. As the proof requires that the (reduced) state space can be chosen compact along this equilibrium, the following sections replace our previous Assumption 4 by the stronger Assumption 5. In addition, the formal arguments in the proofs of Lemma 3.1 and Theorem 2 below assume that the shock space $\Theta$ is finite without explicit notice. These restrictions allow us to directly use the 
characterization of Pareto-inefficiency along with Proposition 4 from Barbie, Hagedorn \& Kaul (2007). While we are confident that an extension of these results to infinite shock spaces would, in principle, be possible and could be obtained along the lines of the proof of Proposition 1 in Barbie \& Kaul (2015), we decided to restrict ourselves here to results that are already available. All other arguments and proofs in this section are formulated and hold also for the general case where $\Theta$ is an interval.

In this section, define $w_{\max }$ by (10) and $w_{\min }$ by (11). As a notational convention, a superscript * identifies functions associated with the ME constructed in Theorem 1 .

\subsection{Pareto optimality}

Our concept of Pareto optimality corresponds to Interim Pareto Optimality (IPO) as defined and studied, e.g., in Demange \& Laroque (2000) or Conditional Pareto Optimality (CPO) as in Chattopadhyay \& Gottardi (1999). The following definition formalizes this concept for the class of economies $\mathscr{E}_{d}$ defined above for a fixed value $d \geq 0$.

\section{Definition 4}

(i) Given $x_{0}=\left(k_{0}, \theta_{0}\right) \in \mathbb{X}$, a feasible allocation of $\mathscr{E}_{d}$ is an adapted stochastic process $a=\left\{k_{t+1}, c_{t}^{y}, c_{t}^{o}\right\}_{t \geq 0}$ with values in $\mathbb{R}_{+}^{3}$ which satisfies the resource constraint

$$
k_{t+1}+c_{t}^{y}+c_{t}^{o}=f\left(k_{t}, \theta_{t}\right)+d
$$

for all $t \geq 0$. The set of feasible allocations of $\mathscr{E}_{d}$ is denoted $\mathbb{A}_{d}\left(x_{0}\right)$.

(ii) Allocation $a \in \mathbb{A}_{d}\left(x_{0}\right)$ (Pareto) dominates allocation $\tilde{a} \in \mathbb{A}_{d}\left(x_{0}\right)$ if $c_{0}^{o} \geq \tilde{c}_{0}^{o}$ and

$$
u_{t}:=\mathbb{E}_{t}\left[u\left(c_{t}^{y}\right)+v\left(c_{t+1}^{o}\right)\right] \geq \mathbb{E}_{t}\left[u\left(\tilde{c}_{t}^{y}\right)+v\left(\tilde{c}_{t+1}^{o}\right)\right]=: \tilde{u}_{t}
$$

for all $t \geq 0$ and for some $t \geq 0$ there exists a non-empty set $A \in \mathcal{F}_{t}$ such that $u_{t}(\omega)>\tilde{u}_{t}(\omega)$ for all $\omega \in A$.

(iii) Allocation $a \in \mathbb{A}_{d}\left(x_{0}\right)$ is called Pareto optimal or efficient if it is not dominated by any other allocation in $\mathbb{A}_{d}\left(x_{0}\right)$. Otherwise, it is called inefficient.

(iv) Allocation $a \in \mathbb{A}_{d}\left(x_{0}\right)$ is called dynamically efficient if there is no other allocation $\tilde{a} \in \mathbb{A}_{d}\left(x_{0}\right)$ such that

$$
c_{t}:=\theta_{t} f\left(k_{t}\right)-k_{t+1} \leq \theta_{t} f\left(\tilde{k}_{t}\right)-\tilde{k}_{t+1}=: \tilde{c}_{t}
$$

for all $t \geq 0$ and for some $t \geq 0$ there exists a non-empty set $A \in \mathcal{F}_{t}$ such that $c_{t}(\omega)<\tilde{c}_{t}(\omega)$ for all $\omega \in A$.

An allocation which is not dynamically efficient as in (iv) is called dynamically inefficient. This type of inefficiency corresponds to an overaccumulation of capital. In such 
cases, it is possible to increase aggregate consumption in each period under any realization of shocks by reducing capital accumulation. Dynamic efficiency therefore excludes this possibility. The concept was originally introduced in Cass (1972) for the deterministic case and extended to the stochastic case by Zilcha (1990). While dynamic inefficiency implies Pareto inefficiency, the converse is not true: Pareto efficiency requires, in addition, that rolling over a state contingent Ponzi scheme is impossible. Intuitively, this excludes cases where an essentially worthless asset is valued because it enhances the possibilities to share risks between successive generations. For this reason, dynamically efficient allocations which are Pareto inefficient will be referred to as allocations with inefficient risk sharing. The relation between these concepts for competitive equilibrium allocations has been examined in Barbie, Hagedorn \& Kaul (2007). In Section 3.5 we present an example of an equilibrium allocation with inefficient risk-sharing.

Our main result in Theorem 2 below establishes that the benchmark economy $\mathscr{E}=\mathscr{E}_{0}$ has a BME whenever the bubbleless equilibrium allocation is Pareto inefficient. To state this result formally, we introduce the concept of a Markovian equilibrium allocation.

\subsection{Markovian equilibrum allocations (MEA)}

For fixed $d \geq 0$, identify a ME of $\mathscr{E}_{d}$ with the mappings $(K, P)$ on $\left.\left.\mathbb{W}=\right] 0, w_{\max }\right]$ constructed as in the previous sections (here and in the sequel we drop the subscript $d$ when convenient). We seek to derive the induced mappings which determine the consumption process along a ME. It will be convenient to define these mappings on the reduced state space $\mathbb{W}$ rather than $\mathbb{X}$ and to identify the state at time $t$ by $w_{t}$. For this reason, we fix the realization of the initial shock $\theta_{0} \in \Theta 9$ and define the consumption mappings associated with a $\mathrm{ME}(K, P)$ as

$$
\begin{aligned}
& C^{y}: \mathbb{W} \longrightarrow \mathbb{R}_{++}, \quad C^{y}(w) \quad:=w-K(w)-P(w) \\
& C^{o}: \mathbb{W} \times \Theta \longrightarrow \mathbb{R}_{++}, \quad C^{o}(w, \theta):=P(W(K(w), \theta))+d+K(w) R(K(w), \theta) .
\end{aligned}
$$

We call the triple $A=\left(K, C^{y}, C^{o}\right)$ a Markovian Equilibrium Allocation (MEA). The pricing kernel associated with $A$ is defined as the map $m_{A}: \mathbb{W} \times \Theta \longrightarrow \mathbb{R}_{++}$,

$$
m_{A}(w, \theta):=\frac{v^{\prime}\left(C^{o}(w, \theta)\right)}{u^{\prime}\left(C^{y}(w)\right)} .
$$

For each $w_{0} \in \mathbb{W}$, a MEA determines a unique feasible allocation $a^{E}\left(w_{0}\right) \in \mathbb{A}_{d}\left(x_{0}\right)$ where $k_{t+1}=K\left(w_{t}\right), c_{t}^{y}=C^{y}\left(w_{t}\right), c_{t+1}^{o}=C^{o}\left(w_{t}, \theta_{t+1}\right)=C^{o}\left(w_{t}, w_{t+1} / W\left(K\left(w_{t}\right), 1\right)\right.$ for $t \geq 0$ while old-age consumption $c_{0}^{o}$ in $t=0$ follows from the aggregate resource constraint. Consequently, we adopt the following notions of efficiency/inefficiency for MEA.

\footnotetext{
${ }^{9}$ This restriction is necessary because initial old-age consumption $c_{0}^{o}$ can, in general, not be written as a function of $w_{0}$ but requires knowledge of the full initial state $x_{0}$. If $\theta_{0}$ is fixed, there is a one-to one correspondence between $w_{0}$ and the initial state $x_{0}$ and the process $\left\{x_{t}\right\}_{t \geq 0}$ can fully be recovered from $\left\{w_{t}\right\}_{t \geq 0}$ as $k_{t}=K\left(w_{t-1}\right)$ and $\theta_{t}=w_{t} / W\left(k_{t}, 1\right)$ for $t \geq 1$.
} 


\section{Definition 5}

A MEA $A=\left(K, C^{y}, C^{o}\right)$ is called

(i) efficient/inefficient at $w_{0} \in \mathbb{W}$ if $a^{E}\left(w_{0}\right)$ is efficient/inefficient.

(ii) efficient/inefficient on $\overline{\mathbb{W}} \subset \mathbb{W}$ if $A$ is efficient/inefficient at all $w_{0} \in \overline{\mathbb{W}}$.

(iii) efficient/inefficient if it is efficient/inefficient at each $w_{0} \in \mathbb{W} 10$

The previous formulation permits consumption and capital along the ME to be expressed as functions of the (reduced) state process $\left\{w_{t}\right\}_{t \geq 0}$. Given $w_{0} \in \mathbb{W}$, the statistical evolution of this process is determined by a time-invariant transition probability $Q$ (see Appendix B for details). Therefore, the lifetime utility $u_{t}$ of generation $t$ from Definition 4 (ii) also depends exclusively on the state $w_{t}$. Combining results from Barbie, Hagedorn \& Kaul (2007) and Barbie \& Kaul (2015), these properties will allow us to characterize the (in-)efficiency of MEA in terms of mappings defined on a one-dimensional state space which greatly simplifies this characterization. To obtain these results, the following additional restrictions on MEA will be employed.

\section{Definition 6}

Let $A=\left(K, C^{y}, C^{0}\right)$ be a MEA defined as above.

(i) We call $A$ continuous if the mappings $K, C^{y}$, and $C^{o}$ are all continuous.

(ii) We call a subset of the form $\overline{\mathbb{W}}=\left[\underline{w}, w_{\max }\right] \subset \mathbb{W}$ a stable set and $\underline{w}>0$ a lower bound (of $A$ ) if $w \in \overline{\mathbb{W}}$ implies $W(K(w), \theta) \in \overline{\mathbb{W}}$ for all $\theta \in \Theta$.

(iii) We call $A$ bounded, if for each $w_{0} \in \mathbb{W}$ there is some stable set $\overline{\mathbb{W}}$ containing $w_{0}$.

The existence of a stable set excludes cases where the state variable converges to zero under some unfavorable sequence of shocks. A stable set can always be chosen as a compact state space because states in this set stay in it under any realization of shocks. For each $d \geq 0$, denote by $A_{d}^{*}=\left(K_{d}^{*}, C_{d}^{y, *}, C_{d}^{o *}\right)$ the MEA associated with the ME $\left(K_{d}^{*}, P_{d}^{*}\right)$ from Theorem 1. Further, let $A_{0}=\left(K_{0}, C_{0}^{y}, C_{0}^{o}\right)$ be the MEA associated with the bubbleless ME of $\mathscr{E}$ derived in Section 1.4. That is, $K_{0}$ is defined by (7), $C_{0}^{y}(w):=w-K_{0}(w)$, and $C_{0}^{o}(w, \theta):=K_{0}(w) R\left(K_{0}(w), \theta\right)$ for all $w \in \mathbb{W}$ and $\theta \in \Theta$. Note that $A_{0}^{*}$ coincides with $A_{0}$ if and only if $\left(K_{0}^{*}, P_{0}^{*}\right)$ is bubbleless, i.e., $P_{0}^{*}=0$. This observation will play a key role in the next section. Also observe that $A_{0}$ and each $A_{d}^{*}$ are continuous by the results from Section 1.4 and Theorem 10 (ii) and that $A_{0}$ is bounded under the additional restriction from Assumption 5 .

\footnotetext{
${ }^{10}$ Under the additional restrictions from Lemma 3.1 (ii) below, the efficiency properties of $A$ become to some extent independent of the initial state $w_{0}$.
} 


\subsection{A general existence theorem}

We are now in a position to state our main existence result in the following theorem.

\section{Theorem 2}

Let Assumptions 1, 2, 3, and 5 hold. If $A_{0}$ is inefficient, then $\left(K_{0}^{*}, P_{0}^{*}\right)$ defines a $B M E$ of $\mathscr{E}$, i.e., $P_{0}^{*}>0$.

The intuition behind the proof of Theorem 2 is straightforward. Consider a monotonic sequence of strictly positive dividend payments $\left(d_{n}\right)_{n \geq 1}$ which converges to zero. For each $n \geq 1$, construct the $\operatorname{ME}\left(K_{d_{n}}^{*}, P_{d_{n}}^{*}\right)$ of $\mathscr{E}_{d_{n}}$ as in Theorem 1 and denote by $A_{d_{n}}^{*}$ the induced MEA defined as above. It is well-known that each $A_{d_{n}}^{*}$, being an equilibrium allocation of an economy with a dividend-paying asset, is efficient. Intuitively, one would expect that this efficiency also holds in the limit such that the sequence $\left(A_{d_{n}}^{*}\right)_{n \geq 1}$ can not converge to $A_{0}$ if $A_{0}$ is inefficient. Thus, $A_{0} \neq A_{0}^{*}$ which is only possible if $P_{0}^{*}>0$, i.e., $\left(K_{0}^{*}, P_{0}^{*}\right)$ is bubbly 11

We preface the proof of Theorem 2 by the following three lemmata. The first result is a sort of unit root condition, which is used in OLG models with finitely many states to characterize the Pareto optimality of stationary competitive equilibria. The proof of part (i) is an adaption of the results from Barbie, Hagedorn \& Kaul (2007) and Barbie \& Kaul (2015). Part (ii) goes beyond the previous results and provides together with Lemma 3.2 and Lemma 3.3 a complete characterization of inefficiency. This part makes use of our monotonicity assumptions to obtain the more general result. The proofs are relegated to Appendix B. Also note the similarity of (21) to the conditions for inefficiency in Demange \& Laroque (2000) or Magill \& Quinzii (2003).

\section{Lemma 3.1}

Let $A=\left(K, C^{y}, C^{o}\right)$ be a MEA which is continuous and bounded.

(i) If $A$ is inefficient, there is an upper-semi-continuous function $\eta: \mathbb{W} \longrightarrow] 0,1]$ such that

$$
\mathbb{E}_{\nu}\left[\eta(W(K(w), \cdot)) m_{A}(w, \cdot)\right]>\eta(w) \text { for all } w \in \mathbb{W}
$$

(ii) If $m_{A}$ in (20) is increasing, then $\eta$ in (i) can be chosen continuous. Moreover, if $A$ is inefficient at some $w_{0} \in \mathbb{W}$, it is also inefficient for all $w_{0}^{\prime} \geq w_{0}$.

Let $m_{0}:=m_{A_{0}}$ be the pricing kernel associated with the bubbleless allocation $A_{0}$. Our next result ensures that $\eta$ in (21) can be chosen continuous whenever $A_{0}$ is inefficient.

\footnotetext{
${ }^{11}$ The same kind of argument is used in Barbie \& Kaul (2015), going back to the basic idea in Aiyagari \& Peled (1991), for the case of an exchange economy, where instead of the monotonicity methods applied here Schauder's fixed point theorem is used. Since in our framework in addition the capital stock adjusts as an endogenous variable, the analysis becomes more complicated than under pure exchange.
} 


\section{Lemma 3.2}

If Assumptions 1, 2, 3, and 4 hold, then $m_{0}$ is increasing.

Finally, we have the following sufficient condition for inefficiency. This condition also appears as part of Theorem 1 of Barbie \& Kaul (2015) and as Theorem 1 in Demange \& Laroque (2000). The proof we give here is similar to the ones given in these papers.

\section{Lemma 3.3}

Let $A=\left(K, C^{y}, C^{o}\right)$ be continuous and $\overline{\mathbb{W}}$ be a stable set of $A$. If a continuous function $\eta: \overline{\mathbb{W}} \longrightarrow] 0,1]$ satisfies (21) for all $w \in \overline{\mathbb{W}}$, then $A$ is inefficient on $\overline{\mathbb{W}}$.

We are now in a position to prove Theorem 2 in five steps.

Step 1: Let $w_{0} \in \mathbb{W}$ be arbitrary and $\overline{\mathbb{W}}=\overline{\mathbb{W}}_{A_{0}}=\left[\underline{w}, w_{\max }\right]$ be a stable set of $A_{0}$ containing $w_{0}$ such that $W\left(K_{0}(\underline{w}), \theta_{\min }\right) / \underline{w}>1$. Assumption 5 ensures that such a set exists. By hypothesis, $A_{0}$ is inefficient at $w_{0}$. Thus, invoking Lemmata 3.1 and 3.2 , there exists a continuous function $\eta: \mathbb{W} \rightarrow] 0,1]$ such that for all $w \in \overline{\mathbb{W}}$ :

$$
\mathbb{E}_{\nu}\left[\eta\left(W\left(K_{0}(w), \cdot\right)\right) m_{0}(w, \cdot)\right]>\eta(w) .
$$

Step 2: Define the sequence $\left(d_{n}\right)_{n \geq 1}$ as $d_{n}:=\bar{d} / n$ for $n \geq 1$ with $\bar{d}>0$ specified below. For each $n \geq 1$, let $\left(K_{d_{n}}^{*}, P_{d_{n}}^{*}\right)$ be the ME of $\mathscr{E}_{d_{n}}$ from Theorem 1 and define the induced MEA $A_{d_{n}}^{*}=\left(K_{d_{n}}^{*}, C_{d_{n}}^{y^{*}}, C_{d_{n}}^{o *}\right)$ as in Section [3.2. By Lemma 2.5, the sequence $\left(K_{d_{n}}^{*}, P_{d_{n}}^{*}\right)_{n \geq 1}$ converges pointwise to the $\operatorname{ME}\left(K_{0}^{*}, P_{0}^{*}\right)$ of $\mathscr{E}$ which satisfies either $P_{0}^{*}=0$ or $P_{0}^{*}>0$. We will show that the first case is impossible under the hypotheses of the theorem. Thus, with the aim of obtaining a contradiction, the remainder assumes $P_{0}^{*}=0$ which implies $K_{0}^{*}=K_{0}$. Then, the sequence $\left(A_{d_{n}}^{*}\right)_{n \geq 1}$ converges pointwise to $A_{0}=\left(K_{0}, C_{0}^{y}, C_{0}^{o}\right)$ defined above. Further, the sequence $\left(m_{n}\right)_{n>1}$ of pricing kernels $m_{n}:=m_{A_{d_{n}}^{*}}$ associated with $A_{d_{n}}^{*}$ defined in (20) converges pointwise to $m_{0}=m_{A_{0}}$.

Step 3: We choose $\bar{d}>0$ such that $\overline{\mathbb{W}}=\left[\underline{w}, w_{\max }\right]$ is stable for each $A_{d_{n}}^{*}$. Since $\left(K_{d_{n}}^{*}\right)_{n \geq 1}$ is increasing by Theorem 1 (i), it suffices to specify $\bar{d}$ such that $\overline{\mathbb{W}}$ is stable for $A_{d_{1}}^{*}$. As $\delta:=W\left(K_{0}(\underline{w}), \theta_{\min }\right) / \underline{w}>1$ and $K_{d_{1}}^{*}=K_{\bar{d}}^{*}$ converges pointwise to $K_{0}$ for $\bar{d} \searrow 0$ due to Lemma 2.5, choosing $\bar{d}>0$ small ensures $W\left(K_{\bar{d}}^{*}(\underline{w}), \theta_{\min }\right) / \underline{w}>1$. Then, $w \geq \underline{w}$ implies $W\left(K_{d_{n}}^{*}(w), \theta\right) \geq W\left(K_{d_{1}}^{*}(w), \theta_{\min }\right) \geq W\left(K_{d_{1}}^{*}(\underline{w}), \theta_{\min }\right)>\underline{w}$, i.e., $\overline{\mathbb{W}}$ is stable for $A_{d_{n}}^{*}$.

Step 4: Standard arguments imply that each $A_{d_{n}}^{*}$ is efficient on $\overline{\mathbb{W}}$. To see this, define for $n \geq 1$ the continuous function $R_{n}^{*}(w, \theta):=\left(P_{d_{n}}^{*}\left(W\left(K_{d_{n}}^{*}(w), \theta\right)\right)+d_{n}\right) / P_{d_{n}}^{*}(w)$ which satisfies $\mathbb{E}_{\nu}\left[m_{n}(w, \cdot) R_{n}^{*}(w, \cdot)\right]=1$ for all $w \in \overline{\mathbb{W}}$. Thus, $R_{n}^{*}$ is a return in the sense of Barbie, Hagedorn \& Kaul (2007), cf. their equation (5). For all $T>0$ and $w_{0} \in \overline{\mathbb{W}}$, monotonicity of $P_{d_{n}}^{*}$ implies $\Pi_{t=1}^{T} R_{n}^{*}\left(w_{t-1}, \theta_{t}\right) \geq P_{d_{n}}^{*}\left(w_{T}\right) / P_{d_{n}}^{*}\left(w_{0}\right) \geq P_{d_{n}}^{*}(\underline{w}) / P_{d_{n}}^{*}\left(w_{\max }\right)=: M$ for any realization of shocks $\theta_{1}, \ldots, \theta_{T}$ where $w_{t}=W\left(K_{d_{n}}^{*}\left(w_{t-1}\right), \theta_{t}\right)$. Note that $M$ is independent of $T$ and the shocks. Using Proposition 4(a) in Barbie, Hagedorn \& Kaul (2007), this implies that $A_{d_{n}}^{*}$ is interim Pareto efficient on $\overline{\mathbb{W}} 12$

\footnotetext{
${ }^{12}$ The result that economies with a dividend paying asset have efficient equilibria is well-known and can also by proved by defining state contingent claims prices and showing that the value of the aggregate
} 
Step 5: Combining the previous result with Lemma 3.3 shows that for each $n \geq 1$ there exists some $w_{n} \in \overline{\mathbb{W}}$ such that

$$
\mathbb{E}_{\nu}\left[\eta\left(W\left(K_{d_{n}}^{*}\left(w_{n}\right), \cdot\right)\right) m_{n}\left(w_{n}, \cdot\right)\right] \leq \eta\left(w_{n}\right) .
$$

Since $\overline{\mathbb{W}}$ is compact, the sequence $\left(w_{n}\right)_{n \geq 1}$ contains a subsequence converging to some $w^{*} \in \mathbb{W}$. Denote this sequence again by $\left(w_{n}\right)_{n \geq 1}$. Clearly, $\lim _{n \rightarrow \infty} \eta\left(w_{n}\right)=\eta\left(w^{*}\right)$ by continuity of $\eta$. We would like to show that for all $\theta \in \Theta$

$$
\lim _{n \rightarrow \infty} \eta\left(W\left(K_{d_{n}}^{*}\left(w_{n}\right), \theta\right)\right) m_{n}\left(w_{n}, \theta\right)=\eta\left(W\left(K_{0}\left(w^{*}\right), \theta\right)\right) m_{0}\left(w^{*}, \theta\right) .
$$

Since all functions in (24) are continuous, it suffices to show that $\lim _{n \rightarrow \infty} P_{d_{n}}^{*}\left(w_{n}\right)=0$ and $\lim _{n \rightarrow \infty} K_{d_{n}}^{*}\left(w_{n}\right)=K_{0}\left(w^{*}\right)$. We have that $\lim _{n \rightarrow \infty} \sup \left\{P_{d_{n}}^{*}(w) \mid w \in \overline{\mathbb{W}}\right\}=0$ by Theorem A in Buchanan \& Hildebrandt (1908)13, which immediately gives the result for $P_{d_{n}}^{*}$. Also by Theorem A in Buchanan \& Hildebrandt (1908), $\left(K_{d_{n}}^{*}\right)_{n \geq 1}$ being a sequence of strictly monotonic functions converges uniformly to $K_{0}$ on the compact interval $\overline{\mathbb{W}}$. Combined with continuity of $K_{0}$, for any $\delta>0$ there exists $n_{0}$ such that $n>n_{0}$ implies

$$
\left|K_{d_{n}}^{*}\left(w_{n}\right)-K_{0}\left(w^{*}\right)\right| \leq\left|K_{d_{n}}^{*}\left(w_{n}\right)-K_{0}\left(w_{n}\right)\right|+\left|K_{0}\left(w_{n}\right)-K_{0}\left(w^{*}\right)\right|<\delta .
$$

This proves (24). Further, $\eta$ is bounded as a continuous function on the compact set $\overline{\mathbb{W}}$ while $0<m_{n}\left(w_{n}, \theta\right)<v^{\prime}\left(K_{\bar{d}}(\underline{w}) R\left(K_{\bar{d}}(\underline{w}), \theta_{\min }\right)\right) / u^{\prime}\left(w_{\max }\right)$ for each $\theta \in \Theta$. Thus, by the Lebesgue-dominated convergence theorem, (23) and (24) imply

$$
\mathbb{E}_{\nu}\left[\eta\left(W\left(K_{0}\left(w^{*}\right), \cdot\right)\right) m_{0}\left(w^{*}, \cdot\right)\right] \leq \eta\left(w^{*}\right) .
$$

But this contradicts (22) and proves the claim that $P_{0}^{*}>0$.

The previous construction also suggests that the limiting MEA $A_{0}^{*}$ associated with the $\operatorname{BME}\left(K_{0}^{*}, P_{0}^{*}\right)$ is efficient. Clearly, if $A_{0}^{*}$ is bounded, this follows immediately from the same arguments used in Step 4. We thus have the following result.

\section{Proposition 1}

If the limiting allocation $A_{0}^{*}$ associated with the $B M E\left(K_{0}^{*}, P_{0}^{*}\right)$ constructed above is bounded, then it is efficient.

Unfortunately, however, boundedness of $A_{0}^{*}$ is not guaranteed even if the bubbleless equilibrium satisfies Assumption 5 .

endowment is finite (due to the presence of dividends). Efficiency of the equilibrium allocation then follows along the lines of the standard proof of the first welfare theorem.

${ }^{13}$ Their result states the simple, but in our analysis very useful fact that if a sequence of monotonic real-valued functions $f_{n}$ defined on the interval $[a, b]$ with $a<b$ converges pointwise to a continuous function $f$ on $[a, b]$, then $f$ is also monotonic and convergence is uniform. 
Under the hypotheses of Theorem 2, suppose the shock process is non-degenerate, i.e., $\theta_{\min }<\theta_{\max }$ or, equivalently, $w_{\min }<w_{\max }$ defined by (10) and (11). Then, for any initial value $w_{0} \in \mathbb{W}$, the dynamics (9) takes values in the ergodic set $\left[w_{\min }, w_{\text {max }}\right]$ after finitely many periods with positive probability. In this case, global inefficiency of $A_{0}$ is equivalent to inefficiency on the ergodic set which, by Lemma 3.1 (ii) and 3.2 is equivalent to $A_{0}$ being inefficient at $w_{\text {min }}$. Thus, we obtain the following existence result as a corollary to Theorem 2 ,

\section{Corollary 3.1}

Let Assumptions 1, 2, 3, and 5 hold. If $w_{\min }<w_{\max }$ and $A_{0}$ is inefficient at $w_{\min }$, then $\left(K_{0}^{*}, P_{0}^{*}\right)$ is a $B M E$ of $\mathscr{E}$, i.e., $P_{0}^{*}>0$.

\subsection{Conditions for inefficiency of $A_{0}$}

In this section we provide necessary and sufficient conditions for $A_{0}$ to be inefficient as required in Theorem 1 which are simple and easy to verify. As in the previous section, we impose the stronger Assumption 5 and define $w_{\max }$ by (10) and $w_{\min }$ by (11).

Define the bubbleless MEA $A_{0}=\left(K_{0}, C_{0}^{y}, C_{0}^{o}\right)$ as before. The pricing kernel $m_{0}=m_{A_{0}}$ defined in (201) induces a map $M: \mathbb{W} \longrightarrow \mathbb{R}_{++}$,

$$
M(w):=\mathbb{E}_{\nu}\left[m_{0}(w, \cdot)\right] .
$$

Economically, the value $1 / M(w)$ can be interpreted as the riskless return in state $w \in \mathbb{W}$. Using (7) and the definition (20) of $m_{0}, M$ can equivalently be written as

$$
M(w)=\frac{\mathbb{E}_{\nu}\left[v^{\prime}\left(C_{0}^{o}(w, \cdot)\right)\right]}{\mathbb{E}_{\nu}\left[R\left(K_{0}(w), \cdot\right) v^{\prime}\left(C_{0}^{o}(w, \cdot)\right)\right]}, \quad w \in \mathbb{W}
$$

The representation in (27) reveals directly that $M$ is continuously differentiable and satisfies $0 \leq M(w) \leq 1 / R\left(K_{0}(w) ; \theta_{\text {min }}\right)$ for all $w$. The latter implies $\lim _{w \searrow 0} M(w)=0$.

Our first result states a simple sufficient condition under which $A_{0}$ is inefficient. Note that the additional restrictions (T2) or (U2) are not required here.

\section{Lemma 3.4}

Let Assumptions 1, 2, and 5 hold and define $M$ as in (26). If $M(w)>1$ for all $w \in\left[w_{\min }, w_{\max }\right]$, then $A_{0}$ is inefficient.

Proof: We construct a continuous function $\left.\eta:] 0, w_{\max }\right] \longrightarrow \mathbb{R}_{++}$which satisfies (22) for all $w \in \mathbb{W}$. By Lemma 3.3 , this implies inefficiency of $A_{0}$ on any stable set $\left[\underline{w}, w_{\max }\right]$ which implies inefficiency on $\mathbb{W}$.

Defining $W_{0}^{E}$ as in (9), note that $W_{0}^{E}\left(\cdot ; \theta_{\min }\right)$ is strictly increasing and, therefore, invertible on its range. Denote the inverse by $\Lambda$. By continuity of $M$, there exists $\delta>0$ such 
that $M(w)>1$ for all $w \in\left[w_{\min }-\delta, w_{\max }\right]$. Construct a sequence $\left(w_{n}\right)_{n \geq 0}$ by setting $w_{0}:=w_{\min }-\delta$ and $w_{n}:=\Lambda\left(w_{n-1}\right)=\Lambda^{n}\left(w_{0}\right)$ for $n \geq 1$. Note that $\left(w_{n}\right)_{n \geq 0}$ is strictly decreasing and, due to Assumption 5 , converges to zero.

Now construct $\eta$ as follows. For $w \in\left[w_{0}, w_{\max }\right]$, let $\eta(w) \equiv 1$. Then,

$$
\mathbb{E}_{\nu}\left[\eta\left(W_{0}^{E}(w, \cdot)\right) m_{0}(w, \cdot)\right]=M(w)>1=\eta(w)
$$

for all $w \in\left[w_{0}, w_{\max }\right]$. Second, for $w \in\left[w_{1}, w_{0}\left[\right.\right.$ let $\eta(w):=M(w) / M\left(w_{0}\right)$. Then,

$$
\mathbb{E}_{\nu}\left[\eta\left(W_{0}^{E}(w, \cdot)\right) m_{0}(w, \cdot)\right]=M(w)>M(w) / M\left(w_{0}\right)=\eta(w)
$$

for all $w \in\left[w_{1}, w_{0}\left[\right.\right.$. Now proceed inductively for $n \geq 1$ by defining for $w \in\left[w_{n}, w_{n-1}[\right.$

$$
\eta(w):=\mathbb{E}_{\nu}\left[\eta\left(W_{0}^{E}(w, \cdot)\right) m_{0}(w, \cdot)\right] / M\left(w_{0}\right) .
$$

By construction, $\eta$ is a continuous function which satisfies (22). Since $\left[w_{0}, w_{\max }\right] \cup$ $\left(\cup_{n \geq 1}\left[w_{n}, w_{n-1}[)=\right] 0, w_{\max }\right]$ the previous construction covers the entire interval $\mathbb{W}$.

A partial converse to Lemma 3.4 is the following result.

\section{Lemma 3.5}

Let Assumptions 1, 2, and 5 hold. If $A_{0}$ is inefficient, then $M(w)>1$ for at least one $w \in\left[w_{\min }, w_{\max }\right]$.

Proof: By contradiction, suppose $A_{0}$ is inefficient but $M(w) \leq 1$ for all $w \in\left[w_{\min }, w_{\max }\right]$. By Lemma 3.1, there is an upper-semi-continuous function $\eta: \mathbb{W} \longrightarrow \mathbb{R}_{++}$such that

$$
\mathbb{E}_{\nu}\left[\eta\left(W_{0}^{E}(w, \cdot)\right) m_{0}(w, \cdot)\right]>\eta(w) .
$$

for all $w \in\left[w_{\min }, w_{\max }\right]$. By Theorem 2.43 in Aliprantis \& Border (2007, p.44), $\eta$ attains a maximum on any compact set and the set of maximizers is compact. Letting $w^{*} \in\left[w_{\min }, w_{\max }\right]$ be a value for which $\eta\left(w^{*}\right)=\eta_{\max }:=\max \left\{\eta(w) \mid w \in\left[w_{\min }, w_{\max }\right]\right\}$,

$$
\mathbb{E}_{\nu}\left[\eta\left(W_{0}^{E}\left(w^{*}, \cdot\right)\right) m_{0}\left(w^{*}, \cdot\right)\right] \leq \eta_{\max } \mathbb{E}_{\nu}\left[m_{0}\left(w^{*}, \cdot\right)\right]=\eta_{\max } M\left(w^{*}\right) \leq \eta_{\max }=\eta\left(w^{*}\right)
$$

which is a contradiction.

The previous conditions take an even simpler form under the additional restriction from Assumption 3. In this case, monotonicity of $m_{0}$ due to Lemma 3.2 implies that $M$ is strictly increasing. Combining Lemmata 3.4 and 3.5 then yields the following result.

\section{Theorem 3}

Under Assumptions 1, 2, 3, and 5, the following holds:

(i) If $M\left(w^{\min }\right)>1$, then $A_{0}$ is inefficient.

(ii) If $A_{0}$ is inefficient, then $M\left(w^{\max }\right)>1$. 
In the deterministic case where $w_{\min }=w_{\max }$, the two conditions from Theorem 3 reduce to $M\left(w_{\min }\right)>1$ which is equivalent to a capital return $R<1$ at the bubbleless steady state. This is precisely the condition in Tirole (1985) which is sufficient and necessary in the deterministic case. In the present stochastic case, the condition $M>1$ requires an 'average' capital return less than unity on the ergodic set $\left[w_{\min }, w_{\max }\right]$.

\subsection{Two example economies}

The following examples illustrate the construction of ME of $\mathscr{E}$ developed in Section 2 and the previous conditions under which the ME is bubbly. The first example allows to compute all solutions explicitly and also demonstrates that the condition $M\left(w_{\min }\right)>1$ from Theorem 3 is not necessary for a BME to exist. The second example illustrates our main result that a BME can exist in dynamically efficient economies by constructing an economy in which the bubbleless equilibrium suffers from inefficient risk sharing.

\section{Example 1}

Suppose $f(k)=k^{\alpha}, 0<\alpha<1, u(c)=\log (c)$, and $v(c)=\beta u(c), \beta>0$. This parametrization is widely studied in the literature, cf. Michel \& Wigniolle (2003) or Demange \& Laroque (2000). Rangazas \& Russell (2005) provide a detailed discussion of the (dynamic) efficiency properties of the bubbleless equilibrium allocation in this case.

One verifies directly that Assumptions 1, 2, and 3 hold. The mapping $K_{0}$ associated with the bubbleless ME of $\mathscr{E}$ defined by (17) computes $K_{0}(w)=\frac{\beta}{1+\beta} w$ such that $W_{0}^{E}$ defined in (9) takes the form

$$
W_{0}^{E}(w, \theta)=\theta(1-\alpha)\left(\frac{\beta}{1+\beta} w\right)^{\alpha} .
$$

Direct computations reveal that $W_{0}^{E}\left(\cdot, \theta_{\max }\right)$ has a unique non-trivial fixed point given by $w_{\max }=\left[(1-\alpha) \theta_{\max }(\beta /(1+\beta))^{\alpha}\right]^{\frac{1}{1-\alpha}}$ which is stable. Further, $W_{0}^{E}\left(\cdot, \theta_{\min }\right)$ also has a unique fixed point $w_{\min }=\left[(1-\alpha) \theta_{\min }(\beta /(1+\beta))^{\alpha}\right]^{\frac{1}{1-\alpha}}$ and Assumption 5 is satisfied. For later reference, let $k_{\max }:=K_{0}\left(w_{\max }\right)$ denote the maximum capital stock and $R_{\max }:=$ $R\left(k_{\max }, \theta_{\max }\right)$ the associated maximum capital return. These values compute explicitly as $k_{\max }=\left[\frac{\beta}{1+\beta}(1-\alpha) \theta_{\max }\right]^{\frac{1}{1-\alpha}}$ and $R_{\max }=\frac{1+\beta}{\beta} \frac{\alpha}{1-\alpha}$.

Applying the construction principle from Section 2.3, let $P_{0}=\mathrm{id} \mathbb{W}_{\mathbb{W}}$ and consider the sequence $\left(P_{n}\right)_{n \geq 0}$ defined as $P_{n}=T\left(P_{n-1}\right), n \geq 1$. As $P(w)=\delta w$ implies $T P(w)=$ $\left[R_{\max }+\delta \frac{1+\beta}{\beta}\right]^{-1} P(w)$ for $w \in \mathbb{W}$, the operator $T$ maps linear functions onto linear functions. Thus, each $P_{n}$ is linear and can be computed explicitly as

$$
P_{n}(w)=\frac{w}{R_{\max }^{n}+\frac{1+\beta}{\beta} \sum_{m=0}^{n-1} R_{\max }^{m}}, n \geq 0 .
$$

For each $w \in \mathbb{W}$, the limit $P_{0}^{*}$ defined in (18) is given by

$$
P_{0}^{*}(w)=\left\{\begin{array}{cl}
\left(\frac{\beta}{1+\beta}-\frac{\alpha}{1-\alpha}\right) w & \text { if } R_{\max }<1 \\
0 & \text { otherwise. }
\end{array}\right.
$$


Thus, in this example, the ME constructed is bubbly, if and only if $R_{\max }<1$ which is equivalent to $A_{0}$ being Pareto inefficient. To relate this result to the condition in Theorem 3 (ii), consider the function $M$ defined in (27) which can be computed as

$$
M(w)=\mathbb{E}_{\nu}\left[\left(R\left(K_{0}(w), \cdot\right)\right)^{-1}\right]
$$

One verifies by direct computations that $M\left(w_{\max }\right)=\frac{1}{R_{\max }} \mathbb{E}_{\nu}\left[\theta_{\max } / \operatorname{id}_{\Theta}(\cdot)\right]$ and $M\left(w_{\min }\right)=$ $\frac{1}{R_{\max }} \mathbb{E}_{\nu}\left[\theta_{\min } / \operatorname{id}_{\Theta}(\cdot)\right]$. As $\mathbb{E}_{\nu}\left[\theta_{\max } / \operatorname{id}_{\Theta}(\cdot)\right]>1, R_{\max }<1$ implies $M\left(w_{\max }\right)>1$. On the other hand, one can easily choose a distribution $\nu$ such that $\mathbb{E}_{\nu}\left[\theta_{\min } / \operatorname{id}_{\Theta}(\cdot)\right]<R_{\max }<1$. In this case, the fixed point (29) satisfies $P_{0}^{*}>0$ and $\mathscr{E}$ has a BME but $M\left(w_{\min }\right)<1$.

In the previous example, the ME defined by (18) is bubbly, if and only if $R_{\max }<1$. We remark that the same condition can be used to ensure existence of a BME in the more general case where both $u$ and $v$ display constant relative risk aversion (of the same degree) bounded by unity while $f$ is of the Cobb-Douglas form. Assumptions 1-5 are again satisfied for this example. The condition $R_{\max }<1$ now ensures existence of a linear function $P(w)=\underline{\delta} w, w \in \mathbb{W}, 0<\underline{\delta}<1$ which is a lower bound for $T$ in the sense that $T \underline{P}>\underline{P} \underline{14}$ By the monotonicity properties of $T$, the fixed point in (18) satisfies $P_{0}^{*}>\underline{P}>0$ and, therefore, defines a BME. While sufficient in both previous cases, condition $R_{\max }<1$ is not necessary for a BME to exist, as the next example shows.

\section{Example 2}

The second example constructs an economy in which the bubbleless equilibrium suffers from inefficient risk-sharing, i.e., is dynamically efficient but Pareto inefficient. It is a slightly modified version of an example from Barbie, Hagedorn \& Kaul (2006).

The specification is as follows. There are two shocks $\Theta=\left\{\theta_{\min }, \theta_{\max }\right\}$ with associated probabilities $\nu_{\min }:=\nu\left(\left\{\theta_{\min }\right\}\right)$ and $\nu_{\max }:=\nu\left(\left\{\theta_{\max }\right\}\right)$. Specifically, we choose $\theta_{\min }=1$, $\theta_{\max }=\frac{5}{3}, \nu_{\min }=\frac{2}{3}$, and $\nu_{\max }=\frac{1}{3}$. Second period utility is again logarithmic with no discounting, i.e., $v(c)=\ln c$ such that Assumption 3 holds and the function $M$ defined in (26) is again strictly increasing and of the form (30). First period utility $u$ and production function $f$ will be chosen below such that Assumptions 1 and 2 hold.

Let $\overline{\mathbb{W}}=\left[w_{\min }, w_{\max }\right]$ denote a stable set of the wage dynamics (9) to be specified below and set $k_{\min }:=K_{0}\left(w_{\min }\right)$ and $k_{\max }:=K_{0}\left(w_{\max }\right)$ with $K_{0}$ defined by (17). Define the production function $f$ locally on the interval $\overline{\mathbb{K}}:=\left[k_{\min }, k_{\max }\right]$ as $f(k)=3+\ln k$. As $f\left(k_{\min }\right)>3>k_{\min } f^{\prime}\left(k_{\min }\right)=1$, we can always extend $f$ to a $C^{2}$ function on the entire interval $\left.] 0, k_{\text {max }}\right]$ such that Assumption [1holds. For the previous specification, the factor pricing functions (11) take the form

$$
\begin{aligned}
W(k, \theta) & =\theta[2+\ln k] \\
R(k, \theta) & =\theta / k
\end{aligned}
$$

for each $k \in \overline{\mathbb{K}}$ and $\theta \in \Theta$. Next, we specify $u$ such that the following holds:

\footnotetext{
${ }^{14}$ The proof of this result is straightforward but rather tedious and, therefore, omitted. It is available from the authors upon request.
} 
(a) $k_{\min }=\frac{5}{4}$ is a fixed point of the capital dynamics under the low shock $\theta_{\min }$, i.e., $k_{\text {min }}=K_{0}\left(w_{\min }\right)$ where $w_{\min }:=W\left(k_{\min }, \theta_{\min }\right)$

(b) $k_{\max }=\frac{4}{3}$ is a fixed pint of the capital dynamics under the high shock $\theta_{\max }$, i.e., $k_{\max }=K_{0}\left(w_{\max }\right)$ where $w_{\max }:=W\left(k_{\max }, \theta_{\max }\right)$.

Defining $c_{\min }:=w_{\min }-k_{\min }$ and $c_{\max }:=w_{\max }-k_{\max }$, let $u$ be a utility function satisfying Assumption 2 and the additional two-point restriction $u^{\prime}\left(c_{\min }\right)=\frac{1}{k_{\min }}=\frac{4}{5}$ and $u^{\prime}\left(c_{\max }\right)=\frac{1}{k_{\max }}=\frac{3}{4}$. Clearly, as $c_{\min }<c_{\max }$, such a function can easily be specified and implies the previous fixed point properties (a) and (b). Further, let us assume that $k_{\max }$ is the unique fixed point of $K_{0}\left(W\left(\cdot, \theta_{\max }\right)\right.$ ) (otherwise, redefine $k_{\max }$ to be the smallest such fixed point and the result below will be the same). Then, for each $k_{0} \in \overline{\mathbb{K}}$, the capital dynamics $k_{t+1}=K_{0}\left(W\left(k_{t}, \theta_{\max }\right)\right)$ under the high shock converges to $k_{\max }$.

We now claim that this economy is dynamically efficient on $\overline{\mathbb{K}}$, i.e., for any $k_{0} \in \overline{\mathbb{K}}$ (equivalently, for any $w_{0} \in \overline{\mathbb{W}}$ ). To see this, we can apply the generalized Cass criterion developed in Zilcha (1990) (see also Rangazas \& Russell (2005) and Barbie \& Kaul (2009)). According to this criterion, a sufficient condition for dynamic efficiency of the economy is $R\left(k, \theta_{\max }\right)>1$ for all $k \in \overline{\mathbb{K}}$ or, equivalently, $R\left(k_{\max }, \theta_{\max }\right)>1$ which holds

since $R\left(k_{\max }, \theta_{\max }\right)=\frac{\theta_{\max }}{k_{\max }}=\frac{5}{3} \frac{3}{4}=\frac{5}{4}$. Intuitively, capital productivity in the good state is always high enough such that aggregate consumption can not be increased in each period and under any shock path.

We also claim that this economy is Pareto inefficient on $\overline{\mathbb{W}}$. By Lemma 3.3, it suffices to choose $\eta$ constant and show that $M(w)>1$ for all $w \in \overline{\mathbb{W}}$. Further, all assumptions of Lemma 3.2 hold, so it is enough to show that $M\left(w_{\min }\right)>1$. Using (30) and recalling that $k_{\min }=K_{0}\left(w_{\min }\right)=\frac{5}{4}$, the map $M$ evaluated at $w_{\min }$ satisfies

$$
M\left(w_{\min }\right)=\mathbb{E}_{\nu}\left[1 / R\left(k_{\min }, \cdot\right)\right]=\frac{\nu_{\min } k_{\min }}{\theta_{\min }}+\frac{\nu_{\max } k_{\min }}{\theta_{\max }}=\frac{2}{3} \frac{5}{4}+\frac{1}{3} \frac{5}{4} \frac{3}{5}=\frac{13}{12}>1 .
$$

Alternatively, Pareto inefficiency could be inferred from Theorem 3, noting that Assumption 5 is only needed to guarantee existence of a stable set in this result. Thus, a BME exists for this economy, although there is no capital overaccumulation.

We expect that one can easily extend the previous inefficiency properties to the entire interval $\left.] 0, w_{\max }\right]$ by choosing $u^{\prime}$ and the extension of $f$ such that $k_{\min }$ respectively $w_{\min }$ is the smallest fixed point of the state dynamics.

\subsection{Dynamics along a BME}

Suppose the ME $\left(K_{0}^{*}, P_{0}^{*}\right)$ of $\mathscr{E}$ constructed in Theorem 1 is bubbly, i.e., $P_{0}^{*}>0$. We seek to deduce several qualitative properties of the equilibrium dynamics along a BME. 
Given the initial state $x_{0}=\left(k_{0}, \theta_{0}\right) \in \mathbb{X}$, all equilibrium variables can be expressed as continuous functions of the equilibrium wage process $\left\{w_{t}\right\}_{t \geq 0}$ which evolves as

$$
w_{t+1}=W^{E}\left(w_{t}, \theta_{t+1}\right):=W\left(K_{0}^{*}\left(w_{t}\right), \theta_{t+1}\right) .
$$

It will again be convenient to study (32) rather than the mathematically equivalent capital dynamics generated by $K^{E}=K_{0}^{*} \circ W$.

As $P_{0}^{*}>0$ implies $K_{0}^{*}<K_{0}$, a first observation is that $W^{E}<W_{0}^{E}$ where the latter is defined in (91). Thus, the sequence generated by (32) is bounded by the wage process (9) along the bubbleless equilibrium under any path of the shock process $\left\{\theta_{t}\right\}_{t \geq 0}$.

A second observation that follows from the Euler equations $(3 \mathrm{a}, \mathrm{b})$ is that in each period the return on the bubbly asset must (weakly) exceed the capital return (1b) in at least one future state. Thus, for each $w \in \mathbb{W}$ there exists some $\theta^{\prime} \in \Theta$ such that

$$
\frac{P_{0}^{*}\left(W^{E}\left(w, \theta^{\prime}\right)\right)}{P_{0}^{*}(w)} \geq R\left(K_{0}^{*}(w), \theta^{\prime}\right)
$$

As $\lim _{w \rightarrow 0} R\left(K_{0}^{*}(w), \theta^{\prime}\right)=\infty$ for all $\theta^{\prime} \in \Theta$ and the left side in (33) is increasing in the shock, there exists a lower bound $\underline{w}^{\prime}>0$ such that $R\left(K_{0}^{*}(w), \theta_{\min }\right)>1$ and $P_{0}^{*}\left(W^{E}\left(w, \theta_{\max }\right)\right)>P_{0}^{*}(w)$ for all $w \leq \underline{w}^{\prime}$. Thus, by monotonicity of $P_{0}^{*}$

$$
W^{E}\left(w, \theta_{\max }\right)>w
$$

for all $w \leq \underline{w}^{\prime}$. As $W^{E}\left(w_{\max }, \theta_{\max }\right)<W_{0}^{E}\left(w_{\max }, \theta_{\max }\right)=w_{\max }$, (34) also shows that $W^{E}\left(\cdot, \theta_{\max }\right)$ has at least one stable fixed point which lies in the interval $] \underline{w}^{\prime}, w_{\max }[$. In

fact, since $\theta_{\max }$ belongs to the support of $\nu$, (34) and continuity of $W^{E}$ imply that for each $w \leq \underline{w}^{\prime}$ there exists a measurable set $\Theta_{w} \subset \Theta$ of positive measure $\nu\left(\Theta_{w}\right)>0$ such that $W^{E}(w, \theta)>w$ holds for all $\theta \in \Theta_{w}$. Thus, defining $\underline{p}^{*}:=P_{0}^{*}\left(\underline{w}^{\prime}\right)$, one observes that the bubbly asset price process $\left\{p_{t}\right\}_{t \geq 0}$ along the BME is persistent in the sense that whenever $p_{t}<\underline{p}^{*}$ there is a positive probability that $p_{t+n}>\underline{p}^{*}$ for some finite $n \geq 1$.

An open question is whether this last result can be strengthened in the sense that $p_{t}<\underline{p}^{*}$ implies $p_{t+n}>p^{*}$ for some finite $n \geq 1$ with probability one. Essentially, this holds when the wage dynamics (32) admits a uniform lower bound $\underline{w}^{\prime}>0$ such that $W^{E}(w, \theta)>w$ for all $\theta \in \Theta$ whenever $w \leq \underline{w}^{\prime}$. The example from Section 3.5 satisfies this condition. If such a lower bound exists, the bubble price processes and in fact all equilibrium variables remain bounded away from zero with probability one. Clearly, Assumption 5 is a necessary precondition for this to hold, but is it sufficient? This question becomes particularly relevant for studying the existence of stationary distributions associated with the state process defined by (32) which we leave for future research.

\subsection{Stochastically bursting bubbles}

By construction, the bubble along a BME never bursts. Asset bubbles which have a constant probability of bursting in each period were first studied in Weil (1987) using 
an otherwise deterministic framework similar to Tirole (1985). It is now straightforward to modify the previous setting along these lines to study ME with bursting bubbles in our setup as well.

Setting $d=0$, suppose that in each period $t$, there is a constant exogenous probability for the bubble to burst in the following period. Thus, the future selling price of the bubbly asset in period $t$ is $p_{t+1}$ with some probability $q$ and zero otherwise. Under this modification, the equilibrium mappings (14a) and (14b) now take the following form (with $q$ replacing the dividend parameter $d=0$ )

$$
\begin{aligned}
H^{1}(k, p ; w, P, q)= & u^{\prime}(w-p-k)-q \mathbb{E}_{\nu}\left[R(k, \cdot) v^{\prime}(P(W(k, \cdot))+k R(k, \cdot))\right] \\
& -(1-q) \mathbb{E}_{\nu}\left[R(k, \cdot) v^{\prime}(k R(k, \cdot))\right] \\
H^{2}(k, p ; w, P, q)= & u^{\prime}(w-p-k) p \\
& -q \mathbb{E}_{\nu}\left[(P(W(k, \cdot))) v^{\prime}(P(W(k, \cdot))+k R(k, \cdot))\right] .
\end{aligned}
$$

The case studied in the previous sections is recovered by setting $q=1$.

It seems now straightforward to repeat the entire analysis of Section 2 based on (35a ) and (35b) rather than (14a) and (14b) and construct such modified Markov equilibria for $q<1$ as fixed points of some operator $T_{q}: \mathscr{G} \longrightarrow \mathscr{G}$ defined by (35a) and (35b) in the exact same fashion as before. As establishing the rather technical Lemmata 2.1 2.4 for the case $q<1$ seems quite tedious, we choose not to work through this case here.

We will, however, show that our existence result derived above for $q=1$ does not necessarily imply the existence of a BME for any $q<1$. To show this, we focus on the first example discussed in Section 3.5 where $f(k)=k^{\alpha}, 0<\alpha<1, u(c)=\log (c)$, and $v(c)=\beta u(c), \beta>0$. Clearly, the bubbleless equilibrium is exactly the same as before and is inefficient if and only if $R_{\max }=\frac{1+\beta}{\beta} \frac{\alpha}{1-\alpha}<1$.

Applying the construction principle from Section 2.3 for $T_{q}$, set $P_{0}=\mathrm{id} \mathbb{w}$ and consider the sequence $\left(P_{n}\right)_{n \geq 0}$ defined as $P_{n}=T_{q}\left(P_{n-1}\right), n \geq 1$. In this example, the operator $T_{q}$ again maps linear functions onto linear functions and can be derived explicitly. One verifies that $P(w)=\delta w, \delta \geq 0$ implies $T_{q} P(w)=\left[R_{\max } / q+\delta \frac{1+\beta}{\beta q}\right]^{-1} P(w)$ for $w \in \mathbb{W}$. Thus, each $P_{n}$ is linear and can be computed explicitly as

$$
P_{n}(w)=\frac{w}{\left(R_{\max } / q\right)^{n}+\frac{1+\beta}{\beta q} \sum_{m=0}^{n-1}\left(R_{\max } / q\right)^{m}}, n \geq 0 .
$$

For each $w \in \mathbb{W}$, the pointwise limit $P_{0}^{*}(w):=\lim _{n \rightarrow \infty} P_{n}(w)$ is given by

$$
P_{0}^{*}(w)=\left\{\begin{array}{cl}
\left(\frac{\beta}{1+\beta} q-\frac{\alpha}{1-\alpha}\right) w & \text { if } R_{\max }<q \\
0 & \text { otherwise. }
\end{array}\right.
$$

Thus, in this example a BME with $q<1$ exists if and only if $R_{\max }<q$. While this restriction also requires inefficiency of the bubbleless equilibrium, the probability 
of bursting $1-q$ must, in addition, be sufficiently small. Essentially the same result is obtained in Weil (1987) for a deterministic economy. We conjecture that a similar conclusion also holds in the general case and that only stochastic bubbles which enjoy a sufficiently high level of confidence can occur in Pareto inefficient economies.

\section{Conclusions}

This paper developed a general approach to construct potentially bubbly Markov equilibria for a general class of frictionless OLG economies with stochastic production. Our main result shows that a BME exists whenever the bubbleless equilibrium is inefficient. This type of inefficiency can be the result of an overaccumulation of capital but also due to inefficient risk sharing between consumers. The deterministic result of Tirole (1985) therefore constitutes a special case of our existence theorem. Our existence result also completes the characterization of the equilibrium set for the class of economies studied in Wang (1993) and related papers by providing conditions under which additional bubbly equilibria exist in these models.

To focus on this existence result and keep the technical part bearable, we deliberately limited the underlying class of economies to a setup with i.i.d. TFP shocks and additive consumer utility. We believe that these restrictions are inessential and easy to dispense with at a cost of a more complex structure and notation. Potential extensions of the previous framework include non-additive utility, non-multiplicative and correlated production shocks, and non-classical production technologies. Models with these features were employed, e.g., in Wang (1994), Morand \& Reffett (2007), McGovern, Morand \& Reffett (2013), or Hillebrand (2014) to study the existence and properties of bubbleless ME. Since all these papers rely on methods similar to those employed in this paper, we believe that the previous construction of a BME should be amendable to these extensions. This constitutes a first major objective of future research.

In addition, several issues remain to be studied even within the framework of this paper. For instance, an open question is if the bubbly equilibrium is always efficient and, related to that, whether it constitutes a Pareto improvement relative to the bubbleless equilibrium. The characterization of Pareto optimality developed in Section 3 should be key to answer these questions. Another avenue of future research is whether the state dynamics along the BME converge to a stationary distribution on the endogenous state space. Since our equilibrium mappings are all monotonic, we view the recent results of Kamihigashi \& Stachurski (2014) as tailor-made for studying the existence, uniqueness, and stability of stationary distributions along a BME. 


\section{A Mathematical Appendix}

\section{A.1 Proof of Lemma 1.1}

For convenience, define the numerator in (b) as $D(k):=\mathbb{E}_{\nu}\left[R(k, \cdot) v^{\prime}(k R(k, \cdot))\right]$ which, as shown in the proof of Lemma 2.1 below is strictly decreasing under property (U) from Assumption 2. Conditions (a) and (b) permit to choose a lower bound $\underline{k}>0$ such that $W\left(k, \theta_{\min }\right)>k$ and $H(k):=u^{\prime}\left(W\left(k, \theta_{\min }\right)-k\right)-D(k)<0$ for all $0<k \leq \underline{k}$. Now define $\underline{w}:=W\left(\underline{k}, \theta_{\min }\right)$ and choose an arbitrary value $\left.\left.\hat{w} \in\right] 0, \underline{w}\right]$. By monotonicity of $W$, there exists a unique $\hat{k} \in] 0, \underline{k}]$ such that $W\left(\hat{k}, \theta_{\min }\right)=\hat{w}$. Then, $H(\hat{k})<0$ and the properties of $D$ and $u^{\prime}$ permit to choose a unique $\hat{k}_{1}$ such that $\hat{k}<\hat{k}_{1}<\hat{w}$ and $u^{\prime}\left(\hat{w}-\hat{k}_{1}\right)=D\left(\hat{k}_{1}\right)$ which is equivalent to $\hat{k}_{1}=K_{0}(\hat{w})$ defined by (7). Then, by monotonicity of $W\left(\cdot, \theta_{\text {min }}\right)$,

$$
\hat{w}=W\left(\hat{k}, \theta_{\min }\right)<W\left(\hat{k}_{1}, \theta_{\min }\right)=W\left(K_{0}(\hat{w}), \theta_{\min }\right)=W_{0}^{E}\left(\hat{w}, \theta_{\min }\right) .
$$

Since $\hat{w} \in] 0, \underline{w}]$ was arbitrary, this proves Assumption 5 ,

\section{A.2 Proof of Lemma 1.2}

By (1),$\frac{f(k)}{k f^{\prime}(k)}=\frac{W\left(k, \theta_{\min }\right)}{k R\left(k, \theta_{\min }\right)}+1$. Thus, NLS implies $\liminf _{k \searrow 0} \frac{W\left(k, \theta_{\min }\right)}{k R\left(k, \theta_{\min }\right)}>0$. Using this and the boundary behavior of $R$, we can choose values $\bar{B}>1>\bar{b}>0$ and a lower bound $\underline{k}_{1}>0$ such that $R\left(k, \theta_{\min }\right)>\frac{\bar{B}}{b}$ and $W\left(k, \theta_{\min }\right)>\bar{b} R\left(k, \theta_{\min }\right) k>\bar{B} k$ for all $0<k \leq \underline{k}_{1}$. Clearly, this implies $\liminf _{k \searrow 0} \frac{W\left(k, \theta_{\min }\right)}{k}>\bar{B}$ and condition (a).

To establish (b), suppose first that $u=\beta^{-1} v$. Note that $\liminf _{k \searrow 0} \frac{W\left(k, \theta_{\min }\right)}{k R\left(k, \theta_{\min }\right)}>0$ and $\lim _{k \searrow 0} \frac{k}{k R\left(k, \theta_{\min }\right)}=0$ imply $\liminf _{k \searrow 0} \frac{W\left(k, \theta_{\min }\right)-k}{k R\left(k, \theta_{\min }\right)}>0$. Thus, there exists $0<\underline{k}_{2} \leq \underline{k}_{1}$ and $\bar{b}_{1}>0$ such that for all $0<k \leq \underline{k}_{2}$ we have

$$
W\left(k, \theta_{\min }\right)-k \geq \bar{b}_{1} k R\left(k, \theta_{\min }\right) .
$$

Suppose $\bar{b}_{1} \geq 1$. Then, $u^{\prime}\left(W\left(k, \theta_{\min }\right)-k\right) \leq u^{\prime}\left(k R\left(k, \theta_{\min }\right)\right)$ for all $0<k \leq \underline{k}_{2}$ yields

$$
\liminf _{k \searrow 0} \frac{v^{\prime}\left(k R\left(k, \theta_{\min }\right)\right)}{u^{\prime}\left(W\left(k, \theta_{\min }\right)-k\right)} \geq \frac{1}{\beta}>0 .
$$

Second, suppose $\bar{b}_{1}<1$. From Assumption 2 (which holds for $u=\beta^{-1} v$ ), we infer that

$$
\bar{b}_{1} u^{\prime}\left(\left(W\left(k, \theta_{\min }\right)-k\right)\right) \leq u^{\prime}\left(\bar{b}_{1}^{-1}\left(W\left(k, \theta_{\min }\right)-k\right)\right)
$$

for all $0<k \leq \underline{k}_{2}$. To see this, define for fixed $c>0$ the map $H(a):=a v^{\prime}(a c)$. Then, $H(1)=v^{\prime}(c)$ and $H^{\prime}(a)=v^{\prime}(a c)+a c v^{\prime \prime}(a c) \geq 0$. Thus, $H$ is non-decreasing and $H(a) \geq H(1)$ for all $a>1$. Setting $c=W\left(k, \theta_{\min }\right)-k$ and $a=\bar{b}_{1}^{-1}>1$, this proves (A.2) which, combined with (A.1) gives

$$
\bar{b}_{1} u^{\prime}\left(\left(W\left(k, \theta_{\min }\right)-k\right)\right) \leq u^{\prime}\left(\bar{b}_{1}^{-1}\left(W\left(k, \theta_{\min }\right)-k\right)\right) \leq u^{\prime}\left(k R\left(k, \theta_{\min }\right)\right)
$$


for all $0<k \leq \underline{k}_{2}$ which implies

$$
\liminf _{k \searrow 0} \frac{v^{\prime}\left(k R\left(k, \theta_{\min }\right)\right)}{u^{\prime}\left(W\left(k, \theta_{\min }\right)-k\right)} \geq \frac{\bar{b}_{1}}{\beta}>0 .
$$

Finally, let $f(0)>0$. Then, NLS implies $\lim _{k \searrow 0} W\left(k, \theta_{\min }\right)=\theta_{\min } f(k)\left(1-\frac{k f^{\prime}(k)}{f(k)}\right)>0$ and, therefore, $\lim _{k \searrow 0} u^{\prime}\left(W\left(k, \theta_{\min }\right)-k\right)>0$ in (12). As the numerator diverges, this implies (b).

\section{A.3 Proof of Lemma 2.1}

Let $P \in \mathscr{G}$ be given and $w \in \mathbb{W}$ be arbitrary but fixed. For each $\left.k \in \mathbb{K}=] 0, k_{\max }\right]$ and $\theta \in \Theta$, set $c(k, \theta):=P(W(k, \theta))+k R(k, \theta)$. Define the functions

$$
V(k):=\frac{\mathbb{E}_{\nu}\left[P(W(k, \cdot)) v^{\prime}(c(k, \cdot))\right]}{\mathbb{E}_{\nu}\left[R(k, \cdot) v^{\prime}(c(k, \cdot))\right]}=: \frac{N(k)}{D(k)}, \quad k \in \mathbb{K}
$$

and

$$
S(k):=k+V(k)=\frac{\mathbb{E}_{\nu}\left[c(k, \cdot) v^{\prime}(c(k, \cdot))\right]}{\mathbb{E}_{\nu}\left[R(k, \cdot) v^{\prime}(c(k, \cdot))\right]}=: \frac{M(k)}{D(k)}, \quad k \in \mathbb{K} .
$$

Since $P$ is continuous, so are the mappings $V, N, D, L$, and $S$. The first part of the proof establishes certain monotonicity properties and the boundary behavior of the previously defined functions. First, we will show that $D$ is strictly decreasing and $S$ is strictly increasing. Fixing an arbitrary interior point $k \in \mathbb{K}$, it suffices to show $D(k+\Delta)<D(k)$ and $S(k+\Delta)>S(k)$ for any $0<\Delta \leq k_{\max }-k$. Since $P$ and $W$ and are weakly increasing and $v^{\prime}$ strictly decreasing,

$$
D(k+\Delta) \leq \tilde{D}(\Delta):=\mathbb{E}_{\nu}\left[R(k+\Delta, \cdot) v^{\prime}(\tilde{c}(\Delta, \cdot))\right]
$$

where $\tilde{c}(\Delta, \theta):=P(W(k, \theta))+(k+\Delta) R(k+\Delta, \theta)$. Likewise, property (U) from Assumption 2 implies that the map $a \mapsto(a+b) v^{\prime}(a+b), a>0$ is weakly increasing for any $b \geq 0$. Therefore,

$$
M(k+\Delta) \geq \tilde{M}(\Delta):=\mathbb{E}_{\nu}\left[\tilde{c}(\Delta, \cdot) v^{\prime}(\tilde{c}(\Delta, \cdot))\right] .
$$

This and (A.6) combined shows that

$$
S(k+\Delta) \geq \tilde{S}(\Delta):=\frac{\tilde{M}(\Delta)}{\tilde{D}(\Delta)} .
$$

Since $\tilde{D}(0)=D(k)$ and $\tilde{S}(0)=S(k)$, it suffices to establish monotonicity of $\tilde{D}$ and $\tilde{S}$. The major advantage is that, unlike $L, D$, and $S$, the maps $\tilde{L}, \tilde{D}$, and $\tilde{S}$ are all differentiable. Dropping arguments when convenient, the derivative of $\tilde{D}$ computes

$$
\begin{aligned}
\tilde{D}^{\prime}(\Delta)= & -\frac{E_{f^{\prime}}(k+\Delta)}{k+\Delta} \mathbb{E}_{\nu}\left[R(k+\Delta, \cdot)\left(v^{\prime}(\cdot)-(k+\Delta) R(k+\Delta, \cdot)\left|v^{\prime \prime}(\cdot)\right|\right)\right] \\
& -\mathbb{E}_{\nu}\left[R(k+\Delta, \cdot)^{2}\left|v^{\prime \prime}(\cdot)\right|\right]
\end{aligned}
$$


where we have used that $\partial_{\Delta} \tilde{c}(\Delta, \theta)=R(k+\Delta, \theta)\left(1-E_{f^{\prime}}(k+\Delta)\right)$. Property (U) from Assumption (2) implies that the expectation in the first term is non-negative and, therefore, $\tilde{D}^{\prime}(\Delta)<0$ and monotonicity of $D$. By (A.8), the sign of $\tilde{S}^{\prime}$ is determined by $H(\Delta):=(k+\Delta)\left(\tilde{M}^{\prime}(\Delta) \tilde{D}(\Delta)-\tilde{M}(\Delta) \tilde{D}^{\prime}(\Delta)\right)$. Noting that $\tilde{M}(\Delta) \geq(k+\Delta) \tilde{D}(\Delta)$ and using $\tilde{D}^{\prime}(\Delta)<0$, it suffices to show that $\tilde{M}^{\prime}(\Delta)>(k+\Delta) \tilde{D}^{\prime}(\Delta)$. The derivative of $\tilde{M}$ computes as

$$
\tilde{M}^{\prime}(\Delta)=\left(1-E_{f^{\prime}}(k+\Delta)\right) \mathbb{E}_{\nu}\left[R(k+\Delta, \cdot)\left(v^{\prime}(\cdot)-\tilde{c}(\Delta, \cdot)\left|v^{\prime \prime}(\cdot)\right|\right)\right]
$$

For brevity, define

$$
\begin{aligned}
& A_{1}(\Delta):=\mathbb{E}_{\nu}\left[R(k+\Delta, \cdot)\left(v^{\prime}(\cdot)-\tilde{c}(\Delta, \cdot)\left|v^{\prime \prime}(\cdot)\right|\right)\right] \\
& A_{2}(\Delta):=\mathbb{E}_{\nu}\left[R(k+\Delta, \cdot)\left(v^{\prime}(\cdot)-(k+\Delta) R(k+\Delta, \cdot)\left|v^{\prime \prime}(\cdot)\right|\right)\right] .
\end{aligned}
$$

Note that property (U) from Assumption 2 implies $0 \leq A_{1}(\Delta) \leq A_{2}(\Delta)$. Further, note from (A.9) that $-(k+\Delta) \tilde{D}^{\prime}(\Delta)>E_{f^{\prime}}(k+\Delta) A_{2}(\Delta)$. Using this property and (A.10) gives the desired result

$$
\tilde{M}^{\prime}(\Delta)-(k+\Delta) \tilde{D}^{\prime}(\Delta)>A_{1}(\Delta)+E_{f^{\prime}}(k+\Delta)\left(A_{2}(\Delta)-A_{1}(\Delta)\right)>0 .
$$

Since $\Theta$ is compact and $k \leq k_{\max }$, consumption $c(k, \theta)$ is uniformly bounded from above (e.g., by $\left.c_{\max }:=P\left(w_{\max }\right)+\theta_{\max } f\left(k_{\max }\right)\right)$ and so is $M$ defined in (A.5) (e.g., by $\left.c_{\max } v^{\prime}\left(c_{\max }\right)\right)$. The boundary conditions from Assumptions 1 and 2 then imply

$$
\lim _{k \searrow 0} D(k)=\infty
$$

and

$$
0 \leq \lim _{k \searrow 0} V(k) \leq \lim _{k \searrow 0} S(k)=\lim _{k \searrow 0} \frac{M(k)}{D(k)}=0 .
$$

Having established the properties necessary for the proof, define

$$
G(k ; w):=u^{\prime}(w-S(k))-D(k) .
$$

Then, the desired solution $\tilde{k}$ solves $G(\tilde{k} ; w)=0$. Observe that $G(\cdot ; w)$ is a strictly increasing function which follows from the monotonicity of $S$ and $D$ and $u^{\prime}$. Thus, any zero is necessarily unique. Also observe the boundary behavior $\lim _{k \searrow_{0}} G(k ; w)=-\infty$ due to A.11). By continuity, it suffices to find a $k<w$ such that $G(k ; w) \geq 0$. Suppose $P \equiv 0$. Then the solution is $\tilde{k}=k_{0}:=K_{0}(w)$ defined by (7) and $\tilde{p}=0$. If $P \neq 0$, consider the following two cases. First, $S\left(k_{0}\right) \geq w$. Then, by (A.12) and monotonicity and continuity of $S$, there exists a unique value $0<\hat{k} \leq k_{0}$ such that $S(\hat{k})=w$ which implies $\lim _{k \succ \hat{k}} G(k ; w)=\infty$. Second, suppose $S\left(k_{0}\right)<w$. Then, $\lim _{k \nearrow k_{0}} G(k ; w)=u^{\prime}\left(w-S\left(k_{0}\right)\right)-D\left(k_{0}\right) \geq G_{0}\left(k_{0} ; w\right)=0$ with $G_{0}$ defined by (7). Thus, in either case, there exists a solution $0<\tilde{k} \leq k_{0}<w$. Setting $\tilde{p}=V(\tilde{k})$ completes the proof. 


\section{A.4 Proof of Lemma 2.2}

Let $P \in \mathscr{G}$ be arbitrary. As shown in the previous proof, $T P=V \circ K_{P}$ where $V$ is defined in (A.4) and, for $w \in \mathbb{W}, k=K_{P}(w)$ is the unique solution to $G(k ; w)=0$ defined in (A.13). Clearly, $K_{P}$ is continuous. Note from A.4 that $T P \geq 0, P>0$ implies $T P>0$ and $P=0$ implies $T P=0$. As $G$ in A.13 is increasing in $P$ and $V$, $K_{P} \leq K_{0}$ for all $P$ with strict inequality if $P>0$. By definition of $K_{P}$ and (A.13), $w>S\left(K_{P}(w)\right)>V\left(K_{P}(w)\right)=T P(w)$ for $w \in \mathbb{W}$ which proves $T P<\mathrm{id} \mathbb{W}_{\text {. }}$

To show that $w \mapsto w-T P(w)$ is (even strictly) increasing, let $w \in \mathbb{W}$ be an arbitrary interior point and choose $\Delta>0$ such that $w+\Delta \in \mathbb{W}$. We show that $T P(w+$ $\Delta)<T P(w)+\Delta$. By contradiction, suppose $T P(w+\Delta) \geq T P(w)+\Delta$. Note that $G$ defined in (A.13) is strictly decreasing in $w$ and strictly increasing in $k$ by strict monotonicity of $D$ and $S$. These properties imply that $K_{P}$ is strictly increasing which gives $K_{P}(w+\Delta)>K_{P}(w)$. Further, as shown in the previous proof, the function $D$ defined in (A.5) is strictly decreasing which gives $D\left(K_{P}(w+\Delta)\right)<D\left(K_{P}(w)\right)$. But by (A.13) and our hypothesis

$$
\begin{aligned}
D\left(K_{P}(w+\Delta)\right) & =u^{\prime}\left(w+\Delta-T P(w+\Delta)-K_{P}(w+\Delta)\right) \\
& \geq u^{\prime}\left(w-T P(w)-K_{P}(w+\Delta)\right) \\
& >u^{\prime}\left(w-T P(w)-K_{P}(w)\right) \\
& =D\left(K_{P}(w)\right)
\end{aligned}
$$

which is a contradiction and proves that $w \mapsto w-T P(w)$ is increasing.

Next, we show that $T P$ is increasing. As $T P=V \circ K_{P}$ and we have already shown that $K_{P}$ is strictly increasing, it remains to show that $V$ defined in (A.4) is increasing as well. To avoid trivialities, assume in the remainder that $P>0$. Adjusting the arguments to the case where $P \geq 0$ is straightforward. Let $k \in \mathbb{K}$ be an arbitrary but fixed interior point. We show that $V(k+\Delta) \geq V(k)$ for any $0<\Delta<k_{\max }-k$. By property (U) from Assumption 2, the map $a \mapsto a v^{\prime}(a+b), a>0$ is increasing for any $b \geq 0$. Thus, by monotonicity of $P \circ W$ the numerator in (A.4) satisfies

$$
N(k+\Delta) \geq \tilde{N}(\Delta):=\mathbb{E}_{\nu}\left[P(W(k, \cdot)) v^{\prime}(P(W, k, \cdot)+(k+\Delta) R(k+\Delta, \cdot))\right] .
$$

Furthermore, by equation (A.6) , the denominator in (A.4 satisfies $D(k+\Delta) \leq \tilde{D}(\Delta)$. Thus, defining $\tilde{V}(\Delta):=\frac{\tilde{N}(\Delta)}{\tilde{N}(\Delta)}$, we have $V(k+\Delta) \geq \tilde{V}(\Delta)$ and $\tilde{V}(0)=V(k)$. It therefore suffices to show that $\tilde{V}$ is increasing. Observe that unlike $V, \tilde{V}$ is $C^{1}$ and, by direct computation, the derivative satisfies $\tilde{V}^{\prime}(\Delta) \geq 0$, if and only if

$$
A(\Delta):=(k+\Delta)^{2} \tilde{N}^{\prime}(\Delta) \tilde{D}(\Delta)-(k+\Delta)^{2} \tilde{D}^{\prime}(\Delta) \tilde{N}(\Delta) \geq 0 .
$$

To establish that $A(\Delta) \geq 0$, let $0<\Delta \leq k_{\max }-k$ be arbitrary but fixed and, for the 
sake of brevity, define the non-negative random variables

$$
\begin{aligned}
X & :=(k+\Delta) R(k+\Delta, \cdot)\left|v^{\prime \prime}(P(k, \cdot)+(k+\Delta) R(k+\Delta, \cdot))\right|^{\frac{1}{2}} \\
Y & :=P(k, \cdot)\left|v^{\prime \prime}(P(k, \cdot)+(k+\Delta) R(k+\Delta, \cdot))\right|^{\frac{1}{2}}
\end{aligned}
$$

both defined on the probability space $(\Theta, \mathscr{B}(\Theta), \nu)$. Then, by direct computation again, the derivative of $\tilde{N}$ defined in (A.14) can be expressed as

$$
(k+\Delta) \tilde{N}^{\prime}(\Delta)=-\left(1-E_{f^{\prime}}(k+\Delta)\right) \mathbb{E}_{\nu}[X Y]
$$

while the derivative of $\tilde{D}$ computed in (A.9) satisfies

$$
-(k+\Delta)^{2} \tilde{D}^{\prime}(\Delta)=E_{f^{\prime}}(k+\Delta)(k+\Delta) \tilde{D}(\Delta)+\left(1-E_{f^{\prime}}(k+\Delta)\right) \mathbb{E}_{\nu}\left[X^{2}\right]
$$

Suppose $E_{f^{\prime}}(k+\Delta) \geq 1$. Then, by (A.17) $\tilde{N}^{\prime}(\Delta) \geq 0$ while, as shown in (A.9), $\tilde{D}^{\prime}(\Delta)<$ 0 . Thus, all terms in (A.15) are positive implying $A(\Delta)>0$ in this case. Therefore, the remainder assumes $E_{f^{\prime}}(k+\Delta)<1$. Note from (44) that $\tilde{N}(\Delta) \geq \frac{\mathbb{E}_{\nu}\left[Y^{2}+X Y\right]}{E_{v^{\prime}}^{\max }} \geq \frac{\mathbb{E}_{\nu}[X Y]}{E_{v^{\prime}}^{\max }}$. Using this together with (A.17) and (A.18) in (A.19) gives

$$
A(\Delta) \geq-(k+\Delta) \tilde{D}(\Delta) \mathbb{E}_{\nu}[X Y]\left(1-E_{f^{\prime}}(k+\Delta) \frac{1+E_{v^{\prime}}^{\max }}{E_{v^{\prime}}^{\max }}\right)+B(\Delta)
$$

where

$$
B(\Delta):=\frac{\mathbb{E}_{\nu}\left[Y^{2}+X Y\right] \mathbb{E}_{\nu}\left[X^{2}\right]}{E_{v^{\prime}}^{\max }}\left(1-E_{f^{\prime}}(k+\Delta)\right) .
$$

By Hölder's inequality (see Aliprantis \& Border (2007, p.463 for the special case $p=$ $q=2$ implying $\left.\frac{1}{p}+\frac{1}{q}=1\right)$ ), we have $\mathbb{E}_{\nu}\left[X^{2}\right] \mathbb{E}_{\nu}\left[Y^{2}\right] \geq\left(\mathbb{E}_{\nu}[X Y]\right)^{2}$ which implies $\mathbb{E}_{\nu}\left[Y^{2}+X Y\right] \mathbb{E}_{\nu}\left[X^{2}\right]=\mathbb{E}_{\nu}\left[Y^{2}\right] \mathbb{E}_{\nu}\left[X^{2}\right]+\mathbb{E}_{\nu}[X Y] \mathbb{E}_{\nu}\left[X^{2}\right] \geq \mathbb{E}_{\nu}[X Y] \mathbb{E}_{\nu}[X(Y+X)]$. Further, using (A.16) and the bounds defined in (44) gives $\mathbb{E}_{\nu}[X(X+Y)] \geq E_{v^{\prime}}^{\min }(k+\Delta) \tilde{D}(\Delta)$. Using both results in (A.20) gives

$$
B(\Delta) \geq \frac{(k+\Delta) \tilde{D}(\Delta) \mathbb{E}_{\nu}[X Y] E_{v^{\prime}}^{\min }}{E_{v^{\prime}}^{\max }}\left(1-E_{f^{\prime}}(k+\Delta)\right) .
$$

Finally, using (A.21) in (A.19) gives the desired result

$$
A(\Delta) \geq-\frac{(k+\Delta) \tilde{D}(\Delta) \mathbb{E}_{\nu}[X Y]}{E_{v^{\prime}}^{\max }}\left(E_{v^{\prime}}^{\max }-E_{v^{\prime}}^{\min }-E_{f^{\prime}}(k+\Delta)\left(1+E_{v^{\prime}}^{\max }-E_{v^{\prime}}^{\min }\right) \geq 0\right.
$$

where the last inequality follows from condition (5) in Assumption 3. This proves that $\tilde{V}$ is weakly increasing which implies the desired result $V(k+\Delta) \geq \tilde{V}(\Delta) \geq \tilde{V}(0)=V(k)$. Finally, adopting an argument used and proved in Morand \& Reffett (2003, p.1360), monotonicity of $T P$ and $w \mapsto w-T P(w), w \in \mathbb{W}$ imply continuity of $T P$. 


\section{A.5 Proof of Lemma 2.3}

Let $P \in \mathscr{G}^{\prime}$ be arbitrary. We need to show that $T P$ is $C^{1}$. Since $P$ is $C^{1}$, so are the mappings $\tilde{P}, S, D$, and $\tilde{N}$ defined in (A.4) and (A.5) and $G$ defined in (A.13). Recall that for each $w \in \mathbb{W}, K_{P}$ determines the unique zero of $G(\cdot ; w)$. Since $\partial_{k} G(k ; w)>0$, $K_{P}$ is $C^{1}$ by the implicit function theorem. Thus, $T P=\tilde{P} \circ K_{P}$ is $C^{1}$ as well.

\section{A.6 Proof of Lemma 2.4}

We only prove the strict inequalities, as the proof of the weak inequalities is analogous. Given $P_{1}, P_{0} \in \mathscr{G}^{\prime}$, suppose $P_{1}>P_{0}$. For $\lambda \in[0,1]$, define $P_{\lambda}:=\lambda P_{1}+(1-\lambda) P_{0}$. Since $\mathscr{G}^{\prime}$ is convex, $P_{\lambda} \in \mathscr{G}^{\prime}$ and, using the monotonicity properties in (13)), the derivative satisfies $0 \leq P_{\lambda}^{\prime} \leq 1$ for all $\lambda$. Moreover, the map $\lambda \mapsto P_{\lambda}=P_{0}+\lambda \Delta$ where $\Delta:=P_{1}-P_{0}>0$ is strictly increasing (with respect to the point-wise ordering on $\mathscr{G}$ ).

Let $w \in \mathbb{W}$ be arbitrary but fixed. By Lemma 2.1 (and a slight abuse of notation), for each $\lambda \in[0,1]$ there exists a unique pair $\left(k_{\lambda}, p_{\lambda}\right)$ which solves $H_{1}\left(k_{\lambda}, p_{\lambda} ; w, \lambda\right)=$ $H_{2}\left(k_{\lambda}, p_{\lambda} ; w, \lambda\right)=0$. We will now show that $\lambda \mapsto k_{\lambda}, \lambda \in[0,1]$ is strictly decreasing and $\lambda \mapsto p_{\lambda}, \lambda \in[0,1]$ is strictly increasing. This implies $p_{1}>p_{0}$ and $k_{1}<k_{0}$ and the claim.

Employing the same definitions and notation as in the proof of Lemma 2.1, write $c_{\lambda}(k, \theta):=P_{\lambda}(W(k, \theta))+k R(k, \theta)$. Then, the pair $\left(k_{\lambda}, p_{\lambda}\right)$ satisfies $p_{\lambda}=\tilde{P}\left(k_{\lambda}, \lambda\right)$ where

$$
\tilde{P}(k, \lambda):=\frac{\mathbb{E}_{\nu}\left[P_{\lambda}(W(k, \cdot)) v^{\prime}\left(c_{\lambda}(k, \cdot)\right)\right]}{\mathbb{E}_{\nu}\left[R(k, \cdot) v^{\prime}\left(c_{\lambda}(k, \cdot)\right)\right]}=: \frac{N(k, \lambda)}{D(k, \lambda)}, \quad k \in \mathbb{K}, \lambda \in[0,1] .
$$

To compute the partial derivatives of $D$ and $N$, note that $\partial_{k} W(k, \theta)=E_{f^{\prime}}(k) R(k, \theta)>0$ by $(1 \mathrm{a}, \mathrm{b})$ which implies

$$
\partial_{k} c_{\lambda}(k, \theta)=R(k, \theta)\left(E_{f^{\prime}}(k) P_{\lambda}^{\prime}(-)+1-E_{f^{\prime}}(k)\right) \geq-R(k, \theta) E_{f^{\prime}}(k) .
$$

Taking the derivative of (A.22) one obtains, exploiting property (U) and suppressing arguments when convenient

$$
\begin{aligned}
\partial_{k} N(k, \lambda) & =\mathbb{E}_{\nu}\left[P_{\lambda}^{\prime}(\cdot) E_{f^{\prime}}(k) R(k, \cdot) v^{\prime}(\cdot)-P_{\lambda}(\cdot)\left|v^{\prime \prime}(\cdot)\right| \partial_{k} c_{\lambda}(k, \cdot)\right] \\
\partial_{\lambda} N(k, \lambda) & =\mathbb{E}_{\nu}\left[\Delta(k, \cdot)\left(v^{\prime}(\cdot)-P_{\lambda}(W(k, \cdot))\left|v^{\prime \prime}(\cdot)\right|\right)\right]>0 \\
\partial_{k} D(k, \lambda) & =-\frac{1}{k} \mathbb{E}_{\nu}\left[E_{f^{\prime}}(k) R(k, \cdot) v^{\prime}(\cdot)+k R(k, \cdot)\left|v^{\prime \prime}(\cdot)\right| \partial_{k} c_{\lambda}(k, \cdot)\right] \\
\partial_{\lambda} D(k, \lambda) & =-\mathbb{E}_{\nu}\left[\Delta(k, \cdot) R(k, \cdot)\left|v^{\prime \prime}(\cdot)\right|\right]<0
\end{aligned}
$$

where $\Delta(k, \theta):=P_{1}(W(k, \theta))-P_{0}(W(k, \theta))>0$ for all $k \in \mathbb{K}$ and $\theta \in \Theta$. Using (A.23) and property (U) from Assumption 2 in (A.26), we infer that

$$
\partial_{k} D(k, \lambda)<-\frac{E_{f^{\prime}}(k)}{k} \mathbb{E}_{\nu}\left[R(k, \cdot)\left(v^{\prime}(\cdot)-k R(k, \cdot)\left|v^{\prime \prime}(\cdot)\right|\right)\right] \leq 0 .
$$


We show that $\frac{d k_{\lambda}}{d \lambda}<0$. As $k_{\lambda}$ is the unique solution to $G(k, \lambda):=u^{\prime}(w-k-\tilde{P}(k, \lambda))-$ $D(k, \lambda)=0$, the implicit function theorem yields the derivative

$$
\frac{d k_{\lambda}}{d \lambda}=-\left.\frac{\partial_{\lambda} G(k, \lambda)}{\partial_{k} G(k, \lambda)}\right|_{k=k_{\lambda}}=-\frac{\left|u^{\prime \prime}(\cdot)\right| \partial_{\lambda} \tilde{P}\left(k_{\lambda}, \lambda\right)-\partial_{\lambda} D\left(k_{\lambda}, \lambda\right)}{\left|u^{\prime \prime}(\cdot)\right|\left(1+\partial_{k} \tilde{P}\left(k_{\lambda}, \lambda\right)\right)-\partial_{k} D\left(k_{\lambda}, \lambda\right)} .
$$

As shown in the proof of Lemma 2.1, the map $S(k, \lambda):=k+\tilde{P}(k, \lambda)$ is strictly increasing in $k$ and, therefore, satisfies $\partial_{k} S(k, \lambda)=1+\partial_{k} \tilde{P}(k, \lambda) \geq 0$. Further, combining (A.22) with (A.25) and (A.27) shows that $\partial_{\lambda} \tilde{P}(k, \lambda)>0$. This together with (A.27) and (A.28) shows that all terms determining the fraction in (A.29) are positive which gives $\frac{d k_{\lambda}}{d \lambda}<0$. Second, we show that $\frac{d p_{\lambda}}{d \lambda}>0$. As $p_{\lambda}=\tilde{P}\left(k_{\lambda}, \lambda\right)$ one obtains the derivative

$$
\frac{d p_{\lambda}}{d \lambda}=\partial_{k} \tilde{P}\left(k_{\lambda}, \lambda\right) \frac{d k_{\lambda}}{d \lambda}+\partial_{\lambda} \tilde{P}\left(k_{\lambda}, \lambda\right)
$$

Using (A.29), the derivative (A.30) can equivalently be written as

$$
\frac{d p_{\lambda}}{d \lambda}=\frac{\left|u^{\prime \prime}(\cdot)\right| \partial_{\lambda} \tilde{P}\left(k_{\lambda}, \lambda\right)+Z\left(k_{\lambda}, \lambda\right)}{\left|u^{\prime \prime}(\cdot)\right|\left(1+\partial_{k} \tilde{P}\left(k_{\lambda}, \lambda\right)\right)-\partial_{k} D\left(k_{\lambda}, \lambda\right)}
$$

where $Z(k, \lambda):=\partial_{\lambda} D(k, \lambda) \partial_{k} \tilde{P}(k, \lambda)-\partial_{k} D(k, \lambda) \partial_{\lambda} \tilde{P}(k, \lambda)$. By (A.26) and our previous result, both the denominator and the first term in the numerator in (A.31) are strictly positive. Hence, it suffices to show that $M\left(k_{\lambda}, \lambda\right) \geq 0$. Using the explicit form of the derivatives $\partial_{k} \tilde{P}$ and $\partial_{\lambda} \tilde{P}$ computed from (A.22), this last expression can be written as

$$
Z(k, \lambda)=\frac{\partial_{\lambda} D(k, \lambda) \partial_{k} N(k, \lambda)-\partial_{k} D(k, \lambda) \partial_{\lambda} N(k, \lambda)}{D(k, \lambda)} .
$$

Using property (U), (A.25), and (A.27) gives $\partial_{\lambda} N(k, \lambda) \geq-k \partial_{\lambda} D(k, \lambda)$. Thus, it suffices to show $\partial_{k} N(k, \lambda)+k \partial_{k} D(k, \lambda) \leq 0$. By (A.24) and (A.26), recalling that $0 \leq P_{\lambda}^{\prime} \leq 1$,

$$
\begin{aligned}
-\partial_{k} N(k, \lambda)-k \partial_{k} D(k, \lambda) & =\mathbb{E}_{\nu}\left[\left(1-P_{\lambda}^{\prime}(\cdot)\right) E_{f}^{\prime}(k) R(k, \cdot) v^{\prime}(\cdot)+c_{\lambda}(k, \cdot)\left|v^{\prime \prime}(\cdot)\right| \partial_{k} c_{\lambda}(k, \cdot)\right] \\
& >E_{f}^{\prime}(k) \mathbb{E}_{\nu}\left[\left(1-P_{\lambda}^{\prime}(\cdot)\right) R(k, \cdot)\left(v^{\prime}(\cdot)-c_{\lambda}(k, \cdot)\left|v^{\prime \prime}(\cdot)\right|\right)\right] \geq 0
\end{aligned}
$$

where the last inequality exploits (A.23). This proves $Z\left(k_{\lambda}, \lambda\right)>0$ and the claim.

\section{A.7 Proof of Corollary 2.1}

(i) $T_{d} P_{1}=T\left(P_{1}+d\right) \geq T\left(P_{0}+d\right)=T_{d} P_{0}$. (ii) $T_{d_{1}} P=T\left(P+d_{1}\right) \geq T\left(P+d_{0}\right)=T_{d_{0}} P$.

\section{A.8 Proof of Theorem 1}

(i) We show the fixed point property for $d=0$. The proof for $d>0$ is analogous. For convenience, we drop the subscript $d=0$ and denote the sequence $\left(T^{n} P_{0}\right)_{n \geq 0}$ simply 
as $\left(P_{n}\right)_{n \geq 0}$ and its pointwise limit by $P^{*}$. Also, for the sake of brevity we abuse our notation by writing $P(k, \theta)$ instead of $P(W(k, \theta))$.

Let $w \in \mathbb{W}$ be arbitrary but fixed. As $\left(P_{n}\right)_{n}$ is a decreasing sequence of functions in $\mathscr{G}^{\prime}$, monotonicity of $K$. due to Lemma 2.4 implies that the sequence $k_{n}:=K_{P_{n}}(w), n \geq 0$ is strictly increasing and converges to some limit $0<k^{*} \leq K_{0}(w) \leq k_{\max }$. The claim will follow if we show that $k^{*}$ and $p^{*}:=P^{*}(w)$ satisfy (15), i.e., $H_{1}\left(k^{*}, p^{*} ; w, P^{*}, 0\right)=$ $H_{2}\left(k^{*}, p^{*} ; w, P^{*}, 0\right)=0$. Uniqueness of the solution to (15) then implies $k^{*}=K_{P^{*}}(w)$.

Let $\theta \in\left[\theta_{\min }, \theta_{\max }\right]$ be arbitrary but fixed. We show that $\lim _{n \rightarrow \infty} P_{n}\left(k_{n}, \theta\right)=P^{*}\left(k^{*}, \theta\right)$. As $\left(P_{n}\right)_{n \geq 0}$ is a sequence of increasing functions which converges pointwise to the continuous function $P^{*}$, convergence is uniform on $\overline{\mathbb{W}}:=\left[W\left(k_{0}, \theta_{\min }\right), w_{\max }\right] \subset \mathbb{W}$ by Theorem A in Buchanan \& Hildebrandt (1908). Note that $W\left(k_{n}, \theta\right) \in \overline{\mathbb{W}}$ for $n \geq 0$. Thus, for each $\delta>0$, there is $n_{0} \geq 0$ such that $\left|P_{n}\left(k_{n}, \theta\right)-P^{*}\left(k_{n}, \theta\right)\right|<\delta / 2$ for all $n \geq n_{0}$. Further, by continuity of $W$ and $P^{*}$ there is $n_{0}^{\prime}>0$ such that $n \geq n_{0}^{\prime}$ implies $\left|P^{*}\left(k_{n}, \theta\right)-P^{*}\left(k^{*}, \theta\right)\right|<\delta / 2$. Combining both insights, we have for all $n \geq \max \left\{n_{0}, n_{0}^{\prime}\right\}$ :

$$
\left|P_{n}\left(k_{n}, \theta\right)-P^{*}\left(k^{*}, \theta\right)\right| \leq\left|P_{n}\left(k_{n}, \theta\right)-P^{*}\left(k_{n}, \theta\right)\right|+\left|P^{*}\left(k_{n}, \theta\right)-P^{*}\left(k^{*}, \theta\right)\right|<\delta .
$$

For $\theta \in\left[\theta_{\min }, \theta_{\max }\right]$, define the functions $\phi_{n}^{1}(\theta):=R\left(k_{n}, \theta\right) v^{\prime}\left(P_{n}\left(k_{n}, \theta\right)+k_{n} R\left(k_{n}, \theta\right)\right)$ and $\phi_{n}^{2}(\theta):=P_{n}\left(k_{n}, \theta\right) v^{\prime}\left(P_{n}\left(k_{n}, \theta\right)+k_{n} R\left(k_{n}, \theta\right)\right)$. The previous result and continuity of $v^{\prime}$ and $R$ imply for each $\theta \in\left[\theta_{\min }, \theta_{\max }\right]$

$$
\begin{aligned}
\lim _{n \rightarrow \infty} \phi_{n}^{1}(\theta) & =\phi_{*}^{1}(\theta):=R\left(k^{*}, \theta\right) v^{\prime}\left(P^{*}\left(k^{*}, \theta\right)+k^{*} R\left(k^{*}, \theta\right)\right) \\
\lim _{n \rightarrow \infty} \phi_{n}^{2}(\theta) & =\phi_{*}^{2}(\theta):=P^{*}\left(k^{*}, \theta\right) v^{\prime}\left(P^{*}\left(k^{*}, \theta\right)+k^{*} R\left(k^{*}, \theta\right)\right) .
\end{aligned}
$$

As $\phi_{n}^{1}(\theta)<R\left(k_{1}, \theta_{\max }\right) v^{\prime}\left(k_{1} R\left(k_{\max }, \theta_{\min }\right)\right)$ and $\phi_{n}^{2}(\theta)<w_{\max } v^{\prime}\left(k_{1} R\left(k_{\max }, \theta_{\min }\right)\right)$ for all $n$, the Lebesgue dominated convergence theorem implies $\lim _{n \rightarrow \infty} \mathbb{E}_{\nu}\left[\phi_{n}^{i}(\cdot)\right]=\mathbb{E}_{\nu}\left[\phi_{*}^{i}(\cdot)\right]$, $i=1,2$. This, $\lim _{n \rightarrow \infty} P_{n}(w)=p^{*}$ and $\lim _{n \rightarrow \infty} u^{\prime}\left(w-P_{n}(w)-k_{n}\right)=u^{\prime}\left(w-p^{*}-k^{*}\right)$ imply that (15) is satisfied. Since $w$ was arbitrary, $P^{*}$ is a fixed point of $T$.

That $d>0$ implies $P_{d}^{*}>0$ follows directly from the Euler equations (14a, b) resp. (15).

To prove the stated properties of $P_{0}^{*}$, we show that $P_{0}^{*}(w)=0$ for some $w \in \mathbb{W}$ implies $P_{0}^{*}(w)=0$ for all $w \in \mathbb{W}$. Let $w_{0} \in \mathbb{W}$ be arbitrary and suppose $P_{0}^{*}\left(w_{0}\right)=0$. If $w_{0}=w_{\max }$, the claim follows from monotonicity of $P_{0}^{*}$, so suppose $w_{0}<w_{\max }$. By (14b) and (15),$P_{0}^{*}\left(w_{0}\right)=0$ implies $P_{0}^{*}\left(W\left(K_{P_{0}^{*}}\left(w_{0}\right), \theta\right)\right)=0 \nu$-a.s. As $\theta_{\max }$ is contained in the support of $\nu$, continuity of $P_{0}^{*}$ yields $P_{0}^{*}\left(W\left(K_{0}^{*}\left(w_{0}\right), \theta_{\max }\right)\right)=0$. Moreover, (14a) and (15) imply $K_{0}^{*}\left(w_{0}\right)=K_{0}\left(w_{0}\right)$, the latter being defined by (7). Thus, under Assumption 4, $w_{1}:=W\left(K_{0}^{*}\left(w_{0}\right), \theta_{\max }\right)$ satisfies $w_{1}=W\left(K_{0}\left(w_{0}\right), \theta_{\max }\right)>w_{0}$ and $P_{0}^{*}\left(w_{1}\right)=0$.

Let $w_{1} \leq w_{n}<w_{\max }$ be any value for which $P_{0}^{*}\left(w_{n}\right)=0$. Repeating the previous argument shows that $w_{n+1}:=W\left(K_{0}^{*}\left(w_{n}\right), \theta_{\max }\right)=W\left(K_{0}\left(w_{n}\right), \theta_{\max }\right)>w_{n}$ and $P_{0}^{*}\left(w_{n+1}\right)=0$. Due to Assumption 4, the sequence $\left(w_{n}\right)_{n \geq 1}$ converges monotonically to $w_{\max }$ and $P_{0}^{*}\left(w_{n}\right)=0$ for all $n \geq 1$ implies $P_{0}^{*}\left(w_{\max }\right)=0$ due to continuity of $P_{0}^{*}$. 
The remaining inequalities follow as limits from the monotonicity of $K_{\bullet}$ and $T$. due to Lemma 2.4 and Corollary 2.1 which imply $P_{d}^{m}>P_{d^{\prime}}^{m}$ and $K_{P_{d}^{m}+d}<K_{P_{d^{\prime}}^{m}+d^{\prime}}$ for all $m$ which must (weakly) also hold in the limit. As for each $w \in \mathbb{W}, K_{d}^{*}(w)$ is the unique zero $k$ of $G_{d}(k ; w)=u^{\prime}\left(w-k-P_{d}^{*}(w)\right)-\mathbb{E}_{\nu}\left[R(k, \cdot) v^{\prime}\left(P_{d}^{*}(W(k, \cdot))+d+k R(k, \cdot)\right)\right]$ which is strictly increasing in $d$, the second inequality even holds strictly.

(ii) Follows directly from $P_{d}^{*} \in \mathscr{G}$ as shown in the main text and Lemma 2.2 (ii).

(iii) Follows directly from the previous results and Definitions 1 and 2 .

\section{A.9 Proof of Lemma 2.5}

Let $\left(d_{n}\right)_{n \geq 0}$ be a sequence converging monotonically to zero. For each $n \geq 1$, define $\left(P_{d_{n}}^{m}\right)_{m \geq 1}$ as $P_{0}=\mathrm{id}_{\mathbb{W}}$ and $P_{d_{n}}^{m}=T_{d_{n}}^{m} P_{0} \in \mathscr{G}^{\prime}$ for $m \geq 1$. This sequence is strictly monotonic and converges pointwise to $P_{d_{n}}^{*} \in \mathscr{G}$ defined in (18). It follows from Theorem 1 (i) that the sequence of limits $\left(P_{d_{n}}^{*}\right)_{n \geq 1}$ is decreasing such that the limiting function

$$
P_{0}^{* *}(w):=\lim _{n \rightarrow \infty} P_{d_{n}}^{*}(w)
$$

is well-defined for all $w \in \mathbb{W}$. Denote by $P_{0}^{*}$ the limit in (18) for $d=0$, i.e.,

$$
P_{0}^{*}(w)=\lim _{m \rightarrow \infty} T^{m} P_{0}(w)
$$

for $w \in \mathbb{W}$. We would like to show that $P_{0}^{* *}=P_{0}^{*}$.

As $T_{d}$ is increasing in $d$ by Corollary 2.1, $P_{d_{n}}^{m}=T_{d_{n}}^{m} P_{0} \geq T^{m} P_{0}=P_{0}^{m}$ for all $m$ which implies $P_{d_{n}}^{*} \geq P_{0}^{*}$ for all $n$. Therefore, $P_{0}^{* *} \geq P_{0}^{*}$. We therefore need to show $P_{0}^{* *} \leq P_{0}^{*}$.

Suppose $d_{n}=0$ for all $n \geq n_{0}$. In this case $n \geq n_{0}$ implies $P_{d_{n}}^{m}=T_{d_{n}}^{m} P_{0}=T^{m} P_{0}=P_{0}^{m}$ for all $m \geq 1$ and, therefore, $P_{0}^{* *}=P_{0}^{*}$. The remainder of the proof therefore assumes that the dividend sequence is strictly positive, i.e., $d_{n}>0$ for all $n$ and strictly decreasing.

We first show that $P_{0}^{* *}$ in $\mathrm{A.32}$ ) is independent of the particular dividend sequence. For $i=1,2$, let $\left(d_{n}^{i}\right)_{n \geq 1}$ be a strictly positive sequence converging monotonically to zero. Denote by $P_{0}^{* * i}$ the pointwise limit (A.32) induced by $\left(d_{n}^{i}\right)_{n \geq 1}$. Now, for each $n \geq 1$ there exists $k \geq 0$ such that $d_{n}^{1}>d_{n+m}^{2}$ for all $m \geq k$. By Theorem 11(i), this implies $P_{d_{n}^{1}}^{*} \geq P_{d_{n+m}^{2}}^{*}$ and, therefore, $P_{d_{n}^{1}}^{*}(w) \geq \lim _{m \rightarrow \infty} P_{d_{n+m}^{2}}^{*}(w)=P_{0}^{* *, 2}(w)$ for all $w \in \mathbb{W}$. Since $n$ was arbitrary, $P_{0}^{* *, 1} \geq P_{0}^{* *, 2}$. Reversing the argument gives $P_{0}^{* *, 2} \geq P_{0}^{* *, 1}$.

We show that $P>P_{0}^{* *}$ implies $T P>P_{0}^{* *}$ for any $P \in \mathscr{G}^{\prime}$. As $P_{0}>P_{0}^{* *}$ and $P_{0} \in \mathscr{G}^{\prime}$, we then obtain by simple induction that $T^{m} P_{0}>P_{0}^{* *}$ for all $m$ which proves $P_{0}^{*} \geq P_{0}^{* *}$. Let $P \in \mathscr{G}^{\prime}$ satisfy $P>P_{0}^{* *}$ and $\hat{w} \in \mathbb{W}$ be arbitrary. We show $T P(\hat{w})>P_{0}^{* *}(\hat{w}) 15$ Given $\hat{w}$, define the compact set $\overline{\mathbb{W}}_{\hat{w}}:=\left[W\left(K_{P}(\hat{w}), \theta_{\min }\right), w_{\max }\right] \subset \mathbb{W}$. We will construct

\footnotetext{
${ }^{15}$ If $P_{0}^{* *} \in \mathscr{G}^{\prime}$, this follows trivially by monotonicity of $T$ and the fixed point property $T P_{0}^{* *}=P_{0}^{* *}$ which can be established as in the proof of Theorem 1. Unfortunately, however, we only know $P_{0}^{* *} \in \mathscr{G}$.
} 
a function $\tilde{P} \in \mathscr{G}^{\prime}$ such that $P>\tilde{P}$ on $\overline{\mathbb{W}}_{\hat{w}}$. Noting that only the behavior of $P$ and $\tilde{P}$ on the interval $\overline{\mathbb{W}}_{\hat{w}}$ is relevant to compute $T P(\hat{w})$ and $T \tilde{P}(\hat{w})$, the same arguments as in the proof of Lemma 2.4 can then be used to show $T P(\hat{w})>T \tilde{P}(\hat{w}) 16$

In order to construct such a $\tilde{P}$, set $\delta:=\min _{w \in \overline{\mathbb{W}}_{\hat{w}}}\left\{P(w)-P_{0}^{* *}(w)\right\}>0$. By Theorem A in Buchanan \& Hildebrandt (1908), there exists a $d>0$ such that $\left\|P_{d}^{*}(w)-P_{0}^{* *}(w)\right\|_{\infty}<\frac{\delta}{3}$ on $\overline{\mathbb{W}}_{\hat{w}}$ as $P_{d}^{*}$ converges montonically to $P_{0}^{* *}$ for $d \searrow 0$ (here $\|\cdot\|_{\infty}$ denotes the supremum norm). By the same argument, there exists $m \in \mathbb{N}$ such that $\left\|T_{d}^{m} P_{0}(w)-P_{d}^{*}(w)\right\|_{\infty}<\frac{\delta}{3}$ on $\overline{\mathbb{W}}_{\hat{w}}$ as $\left(T_{d}^{m} P_{0}\right)_{m>0}$ converges pointwise to $P_{d}^{*}$. Define $\tilde{P}:=T_{d}^{m} P_{0}$ and note that $\left\|\tilde{P}-P_{0}^{* *}\right\|_{\infty}<\frac{2 \delta}{3}$ on $\overline{\mathbb{W}}_{\hat{w}}$. Further, $P_{0}^{* *}<T_{\tilde{d}}^{m+1} P_{0}<T_{\tilde{d}} \circ T_{d}^{m} P_{0}$ on $\mathbb{W}$ for any $0<\tilde{d}<d$. Thus, $P_{0}^{* *}<T_{\tilde{d}} \tilde{P}$ for any $\tilde{d}>0$ which implies $P_{0}^{* *} \leq T \tilde{P}$. This last results uses that

$$
\lim _{n \rightarrow \infty} T_{d_{n}} P(w)=T P(w)
$$

for all $P \in \mathscr{G}^{\prime}, w \in \mathbb{W}$ and any monotonic sequence $\left(d_{n}\right)_{n}$ converging to zero 17 Combining these results we get $T P(\hat{w})>T \tilde{P}(\hat{w}) \geqslant P_{0}^{* *}(\hat{w})$ for any $\hat{w} \in \mathbb{W}$.

To show that $\lim _{n \rightarrow \infty} K_{d_{n}}^{*}(w)=K_{0}^{*}(w)$ for each $w \in \mathbb{W}$, note that $\left(K_{d_{n}}^{*}(w)\right)_{n}$ is increasing by Theorem 1(i) and converges to some limit $k^{*} \leq K_{0}(w)$. By the same arguments used in the proof of Theorem 1(i), $k^{*}$ and $p^{*}:=P_{0}^{*}(w)$ satisfy the Euler equations at $P=P_{0}^{*}$ and $d=0$ which implies $k^{*}=K_{P_{0}^{*}}(w)$ by uniqueness of the solution to (15).

\section{B Efficiency and Inefficiency of MEA}

In this appendix, we review the recursive characterization of interim Pareto optimality for stationary exchange economies obtained in Barbie \& Kaul (2015) and adapt their results to characterize the optimality of $\mathrm{ME}$ in a stochastic production economy. As large parts of the analysis holds almost unchanged and requires mainly notational changes, we refer at many places the reader to Barbie \& Kaul (2015) for the details and proofs and just repeat the core facts. To adapt the results, we need the characterization of interim optimality for production OLG models from Barbie, Hagedorn \& Kaul (2007) who extended the pure exchange case in Chattopadhyay \& Gottardi (1999).

\section{B.1 Notation and definitions}

Let $A=\left(K, C^{y}, C^{o}\right)$ be a continuous, bounded MEA defined as in Section 3.2 and $\overline{\mathbb{W}}=\left[\underline{w}, w_{\max }\right]$ be a stable set of $A$. Fixing the initial shock $\theta_{0} \in \Theta$ permits $\overline{\mathbb{W}}$ to be

\footnotetext{
${ }^{16}$ Observe that any convex combination $P_{\lambda}=\lambda P+(1-\lambda) \tilde{P}$ lies between $P$ and $\tilde{P}$. Therefore, by monotonicity of $K_{\bullet}, W\left(K_{P_{\lambda}}(\hat{w}), \theta\right) \in \overline{\mathbb{W}}_{\hat{w}}$ for all $\theta \in\left[\theta_{\min }, \theta_{\max }\right]$.

${ }^{17}$ To see this, fix $w \in \mathbb{W}$ and let $p_{n}:=T_{d_{n}} P(w)$ and $k_{n}:=K_{P+d_{n}}(w)$. By Corollary 2.1 and monotonicity of $K_{\bullet}$, these sequences converge monotonically to values $p^{*} \geq 0$ and $k^{*}>0$, respectively. As $H^{i}\left(k_{n}, p_{n}, w, P, d_{n}\right)=0$ for all $n$ and $i=1,2$, continuity of $H^{i}$ implies $H^{i}\left(k^{*}, p^{*}, w, P, 0\right)=0$. Uniqueness of the solution to (15) implies $p^{*}=T P(w)$ and $k^{*}=K_{P}(w)$.
} 
used as the state space which corresponds to the set $S$ in Barbie \& Kaul (2015). To adapt our notation to their setup, note that any two successive states $w$ and $w^{\prime}$ permit to recover the shock in the second period via $\theta^{\prime}=w^{\prime} / W(K(w), 1)$. Thus, define the (modified) pricing kernel $m: \overline{\mathbb{W}} \times \overline{\mathbb{W}} \longrightarrow \mathbb{R}_{++}$

$$
m\left(w, w^{\prime}\right):=\frac{v^{\prime}\left(C^{o}\left(w, w^{\prime} / W(K(w), 1)\right)\right)}{u^{\prime}\left(C^{y}(w)\right)} .
$$

Denote by $\mathscr{B}(\overline{\mathbb{W}})$ the Borel- $\sigma$ algebra on $\overline{\mathbb{W}}$. As shocks are i.i.d., function $K$ defines a transition probability $Q: \overline{\mathbb{W}} \times \mathscr{B}(\overline{\mathbb{W}}) \longrightarrow[0,1]$

$$
Q(w, G):=\nu(\{\theta \in \Theta \mid W(K(w), \theta) \in G .\}) .
$$

Note that $Q$ has the Feller property since the function $W \circ K$ is continuous. By the change-of variable formula, the inequality (21) can be written as

$$
\int_{\overline{\mathbb{W}}} \eta\left(w^{\prime}\right) m\left(w, w^{\prime}\right) Q\left(w, d w^{\prime}\right)>\eta(w) .
$$

To adapt their formal arguments the remainder follows Barbie, Hagedorn \& Kaul (2007) by assuming that the shock-process is finite-valued, i.e., $\Theta=\left\{\theta_{1}, \ldots, \theta_{N}\right\}$. Thus, if $w_{t} \in \overline{\mathbb{W}}$ is the state in period $t$, the are $n$ successive states $w_{t+1}=W\left(K\left(w_{t}\right), \theta_{t+1}\right)$. If $w^{\prime} \in \overline{\mathbb{W}}$ is a such a successor, we write $w^{\prime} \succ w_{t}$. With this notation, an integral of the form (B.3) can be written as $\sum_{w^{\prime} \succ w} \eta\left(w^{\prime}\right) m\left(w, w^{\prime}\right) Q\left(w, w^{\prime}\right)$.

Given some initial state $w_{0} \in \overline{\mathbb{W}}$, denote by $W^{t}\left(w_{0}\right)$ the set of histories $w^{t}=\left(w_{0}, \ldots, w_{t}\right)$ observed up to time $t$, i.e., $w_{n} \succ w_{n-1}$ for all $n=1, \ldots, t$. Further, let $W^{\infty}\left(w_{0}\right)$ denote the set of all infinite histories $w^{\infty}=\left(w_{t}^{\infty}\right)_{t \geq 0}$, i.e., $w_{t}^{\infty} \succ w_{t-1}^{\infty}$ for all $t \geq 1$ and $w_{0}^{\infty}=w_{0}$. For any infinite path $w^{\infty} \in W^{\infty}\left(w_{0}\right)$, denote by $\left(w^{\infty}\right)^{t}$ the induced history up to time $t \geq 0$ along this path, i.e. $\left(w^{\infty}\right)^{t}=\left(w_{0}^{\infty}, w_{1}^{\infty}, \ldots, w_{t}^{\infty}\right) \in W^{t}\left(w_{0}\right)$.

Similar to Chattopadhyay \& Gottardi (1999), define for each $w^{t} \in W^{t}\left(w_{0}\right)$ the set weights 18

$$
\mathcal{U}\left(w^{t}\right)=\left\{\lambda\left(w^{t}, w^{\prime}\right) \in \mathbb{R}_{+} \mid w^{\prime} \succ w_{t}, \sum_{w^{\prime} \succ w_{t}} \lambda\left(w^{t}, w^{\prime}\right) Q\left(w_{t}, w^{\prime}\right)=1\right\} .
$$

Given some $w_{0} \in \overline{\mathbb{W}}$, define $\mathcal{U}^{\infty}\left(w_{0}\right)$ to be the family of weights $\lambda^{\infty}=\left(\lambda\left(w^{t}, \cdot\right)\right)_{t \geq 1}$ where $w^{t} \in W^{t}\left(w_{0}\right)$ and $\lambda\left(w^{t}, \cdot\right) \in \mathcal{U}\left(w^{t}\right)$ for all $t$.

\footnotetext{
${ }^{18}$ As explained in detail in Barbie \& Kaul (2015), the definition of a weight given in Chattopadhyay \& Gottardi (1999) (and also in Barbie, Hagedorn \& Kaul (2007)) is slightly different from here (and in Barbie \& Kaul (2015)). Because Chattopadhyay \& Gottardi (1999) use an abstract date-event tree setting without objective probabilities, their definition is without probablities, but equivalent to the one given here.
} 


\section{B.2 Recursive characterization of inefficiency}

Barbie, Hagedorn \& Kaul (2007) derive a condition for interim Pareto inefficiency in a stochastic Diamond model. For a MEA $A$ which satisfies the restrictions from Lemma 3.1, the necessary part of this result can be stated as follows.

\section{Lemma B.1}

If $A=\left(K, C^{y}, C^{o}\right)$ is inefficient at $w_{0} \in \overline{\mathbb{W}}$, there exists a family of weights $\lambda^{\infty} \in \mathcal{U}^{\infty}\left(w_{0}\right)$ and a constant $C \geq 0$ such that for each path $w^{\infty} \in W^{\infty}\left(w_{0}\right)$

$$
\sum_{i=0}^{\infty} \prod_{j=0}^{i} \frac{\lambda\left(\left(w^{\infty}\right)^{j}, w_{j+1}^{\infty}\right)}{m\left(w_{j}^{\infty}, w_{j+1}^{\infty}\right)} \leq C
$$

As noted in Barbie \& Kaul (2015), the condition (B.4) can be restated as a minimax problem. The max-part is taking the supremum over all possible paths, the min-part is taking the infimum over all possible weights. For any $w_{0} \in \overline{\mathbb{W}}$, define the value function

$$
J^{*}\left(w_{0}\right):=\inf _{\lambda^{\infty} \in \mathcal{U}^{\infty}\left(w_{0}\right)} \sup _{w^{\infty} \in W^{\infty}\left(w_{0}\right)}\left\{1+\sum_{i=0}^{\infty} \prod_{j=0}^{i} \frac{\lambda\left(\left(w^{\infty}\right)^{j}, w_{j+1}^{\infty}\right)}{m\left(w_{j}^{\infty}, w_{j+1}^{\infty}\right)}\right\} .
$$

The next result follows immediately from Lemma B.1 and (B.5).

\section{Corollary B.1}

If $A$ is inefficient at $w_{0} \in \overline{\mathbb{W}}$, then $J^{*}\left(w_{0}\right)<\infty$.

Following Barbie \& Kaul (2015) we show that (B.5) defines a recursive structure permitting $J^{*}$ to be computed as a fixed point of some operator $Z$. For each $w \in \overline{\mathbb{W}}$, denote the set of all stationary weights

$$
\mathcal{U}(w)=\left\{\lambda\left(w, w^{\prime}\right) \in \mathbb{R}_{+} \mid w^{\prime} \succ w, \sum_{w^{\prime} \succ w} \lambda\left(w, w^{\prime}\right) Q\left(w, w^{\prime}\right)=1\right\} .
$$

Define the operator $Z$ which associates with any nonnegative extended real-valued function $J: \overline{\mathbb{W}} \longrightarrow \mathbb{R}_{+} \cup\{+\infty\}$ the new function $Z J$ defined for all $w \in \overline{\mathbb{W}}$ as

$$
Z J(w):=1+\inf _{\lambda(w, \cdot) \in \mathcal{U}(w)} \sup _{w^{\prime} \succ w}\left\{\frac{\lambda\left(w, w^{\prime}\right)}{m\left(w, w^{\prime}\right)} \cdot J\left(w^{\prime}\right)\right\} .
$$

Note that $Z$ is monotonic, i.e., $J_{1} \geq J_{2}$ implies $Z J_{1} \geq Z J_{2}$. The operator $Z$ can now be used to compute a value function that solves the functional equation (B.6). Construct the sequence $\left(J_{n}\right)_{n \geq 0}$ of functions $J_{n}$ defined on $\overline{\mathbb{W}}$ recursively by setting $J_{0} \equiv 1$ and $J_{n}=Z J_{n-1}$ for $n \geq 1$. For each $w \in \overline{\mathbb{W}}$, define the function

$$
J_{\infty}(w):=\lim _{n \rightarrow \infty} J_{n}(w)
$$


Note that the pointwise limit in (B.7) exists since the sequence $\left(J_{n}\right)_{n \geq 0}$ is increasing. We now have the following result. The proof is the same as in Barbie \& Kaul (2015) for Theorem 1 and Proposition 2 (with the appropriate notational changes).

\section{Lemma B.2}

The function $J_{\infty}$ defined in $(\overline{B .7})$ is a fixed point of $Z$ that coincides with the value function $J^{*}$ defined in (B.5), i.e., $J_{\infty}=Z J_{\infty}=J^{*}$.

\section{B.3 Proof of Lemma 3.1 (i)}

By Corollary B.1, if $A$ is inefficient then $J^{*}\left(w_{0}\right)<\infty$ for all $w_{0} \in \overline{\mathbb{W}}$. Set $\eta(w):=$ $1 / J^{*}(w)$ for $w \in \overline{\mathbb{W}}$. It follows from the same arguments as in the proofs of Proposition 4 and Theorem 2(a) in Barbie \& Kaul (2015) that $\eta$ is a strictly positive, uppersemicontinuous function which takes values in the unit interval (since $J^{*}>1$ ) and satisfies (B.3) for all $w \in \overline{\mathbb{W}}$. As boundedness of $A$ permits to choose the lower bound $\underline{w}$ arbitrarily small, the previous construction of $\eta$ can be extended to the entire interval $\left.\mathbb{W}=] 0, w_{\max }\right]$.

\section{B.4 Proof of Lemma 3.1 (ii)}

In this section we present a new additional sufficient condition under which the function $\eta$ constructed as in the previous subsection is continuous, not just upper-semicontinuous. We will then argue that this condition is satisfied if the kernel $m_{A}$ exhibits the monotonicity property required in Lemma 3.1 (ii). We have the following result:

\section{Lemma B.3}

Suppose $J^{*}=J_{\infty}$ defined in $(\overline{B .7})$ is uniformly bounded on $\overline{\mathbb{W}}$, i.e., there exists a constant $M \geq 0$ such $J^{*}(w) \leq M$ for all $w \in \overline{\mathbb{W}}$. Then $\eta=1 / J^{*}$ is continuous.

Proof: Construct the sequence $\left(J_{n}\right)_{n \geq 0}$ as above by setting $J_{0} \equiv 1$ and $J_{n}=Z J_{n-1}$ for $n \geq 1$. Recall that $J_{1}>1=J_{0}$ and monotonicity of $Z$ imply that $\left(J_{n}\right)_{n \geq 0}$ is strictly increasing, i.e., $J_{n}>J_{n-1}$ for all $n \geq 0$. By Lemma B.2, we know that the pointwise limit $J^{*}$ defined in (B.7) is a fixed point of $Z$. We will show that under the hypotheses of Lemma B.3, $\left(J_{n}\right)_{n \geq 1}$ is a Cauchy sequence in the space of bounded continuous functions on $\overline{\mathbb{W}}$. As this space is complete, the sequence must converge to some bounded continuous function, which coincides with the pointwise limit $J^{*}$.

First, we show that each $J_{n}$ is of the form $J_{n}(w)=1+c_{n}^{*}(w)$ for some continuous function $c_{n}^{*}: \overline{\mathbb{W}} \longrightarrow \mathbb{R}_{+}$. Clearly, this holds trivially for $n=0$ and $c_{0}^{*} \equiv 0$. By induction, suppose $J_{n-1}(w)=1+c_{n-1}^{*}(w)$ for some $n \geq 1$. For each $w \in \overline{\mathbb{W}}$ and $w^{\prime} \succ w$, define the function

$$
\lambda_{n}^{*}\left(w, w^{\prime}\right):=\frac{m\left(w, w^{\prime}\right)}{J_{n-1}\left(w^{\prime}\right)} c_{n}^{*}(w)
$$


where $c_{n}^{*}$ is chosen such that $\sum_{w^{\prime} \succ w} \lambda_{n}^{*}\left(w, w^{\prime}\right) Q\left(w, w^{\prime}\right)=1$ for all $w \in \overline{\mathbb{W}}$, i.e.,

$$
c_{n}^{*}(w):=\left[\sum_{w^{\prime} \succ w} \frac{m\left(w, w^{\prime}\right)}{J_{n-1}\left(w^{\prime}\right)} Q\left(w, w^{\prime}\right)\right]^{-1} .
$$

Note that $\lambda_{n}^{*}$ is continuous and attains the infimum in (B.6). Hence,

$$
J_{n}(w)=1+\max _{w^{\prime} \succ w} \frac{\lambda_{n}^{*}\left(w, w^{\prime}\right)}{m\left(w, w^{\prime}\right)} J_{n-1}\left(w^{\prime}\right)=1+c_{n}^{*}(w) .
$$

As continuity of $c_{n-1}^{*}$ implies continuity of $c_{n}^{*}$, this proves that each $J_{n}$ is continuous and, therefore, bounded on the compact set $\overline{\mathbb{W}}$.

Defining $\lambda_{n}^{*}$ by $(\underline{\mathrm{B} .8})$ for each $n \geq 1$ we can now use the first equality in (B.10) to expand $J_{n}$ for all $w_{0} \in \overline{\mathbb{W}}$ as

$$
\begin{aligned}
J_{n}\left(w_{0}\right)= & 1+\max _{w_{1} \succ w_{0}} \frac{\lambda_{n}^{*}\left(w_{0}, w_{1}\right)}{m\left(w_{0}, w_{1}\right)}\left[1+\max _{w_{2} \succ w_{1}} \frac{\lambda_{n-1}^{*}\left(w_{1}, w_{2}\right)}{m\left(w_{1}, w_{2}\right)} J_{n-2}\left(w_{2}\right)\right] \\
= & 1+\max _{w_{1} \succ w_{0}} \frac{\lambda_{n}^{*}\left(w_{0}, w_{1}\right)}{m\left(w_{0}, w_{1}\right)}\left[1+\max _{w_{2} \succ w_{1}} \frac{\lambda_{n-1}^{*}\left(w_{1}, w_{2}\right)}{m\left(w_{1}, w_{2}\right)}[\ldots\right. \\
& {\left.\left.\left[1+\max _{w_{n} \succ w_{n-1}} \frac{\lambda_{1}^{*}\left(w_{n-1}, w_{n}\right)}{m\left(w_{n-1}, w_{n}\right)}\right] \ldots\right]\right] . }
\end{aligned}
$$

The final term in (B.11) satisfies $1+\max _{w_{n} \succ w_{n-1}} \frac{\lambda_{1}^{*}\left(w_{n-1}, w_{n}\right)}{m\left(w_{n-1}, w_{n}\right)}=J_{1}\left(w_{n-1}\right)=1+c_{1}^{*}\left(w_{n-1}\right)$.

Clearly, $\lambda_{n}^{*}$ does not necessarily attain the infimum when defining $J_{n+1}$ by (B.6) $)$. Therefore, for all $w_{0} \in \overline{\mathbb{W}}$, recalling that $J_{1}(w)=1+c_{1}^{*}(w)$

$$
\begin{aligned}
J_{n+1}\left(w_{0}\right)= & 1+\max _{w_{1} \succ w_{0}} \frac{\lambda_{n+1}^{*}\left(w_{0}, w_{1}\right)}{m\left(w_{0}, w_{1}\right)} J_{n}\left(w_{1}\right) \\
\leq & 1+\max _{w_{1} \succ w_{0}} \frac{\lambda_{n}^{*}\left(w_{0}, w_{1}\right)}{m\left(w_{0}, w_{1}\right)} J_{n}\left(w_{1}\right) \\
\leq & 1+\max _{w_{1} \succ w_{0}} \frac{\lambda_{n}^{*}\left(w_{0}, w_{1}\right)}{m\left(w_{0}, w_{1}\right)}\left[1+\max _{w_{2} \succ w_{1}} \frac{\lambda_{n-1}^{*}\left(w_{1}, w_{2}\right)}{m\left(w_{1}, w_{2}\right)}[\ldots\right. \\
& \left.\left.1+\max _{w_{n-1} \succ w_{n-2}} \frac{\lambda_{2}^{*}\left(w_{n-2}, w_{n-1}\right)}{m\left(w_{n-2}, w_{n-1}\right)}\left[1+\max _{w_{n} \succ w_{n-1}} \frac{\lambda_{1}^{*}\left(w_{n-1}, w_{n}\right)}{m\left(w_{n-1}, w_{n}\right)}\left(1+c_{1}^{*}\left(w_{n}\right)\right)\right] \ldots\right]\right] .
\end{aligned}
$$


By elementary observations 19 , the final term in (B.12) satisfies for any $w_{n-2} \in \overline{\mathbb{W}}$

$$
\begin{aligned}
& \max _{w_{n-1} \succ w_{n-2}} \frac{\lambda_{2}^{*}\left(w_{n-2}, w_{n-1}\right)}{m\left(w_{n-2}, w_{n-1}\right)}\left[1+\max _{w_{n} \succ w_{n-1}} \frac{\lambda_{1}^{*}\left(w_{n-1}, w_{n}\right)}{m\left(w_{n-1}, w_{n}\right)}\left(1+c_{1}^{*}\left(w_{n}\right)\right)\right] \\
\leq & \max _{w_{n-1} \succ w_{n-2}} \frac{\lambda_{2}^{*}\left(w_{n-2}, w_{n-1}\right)}{m\left(w_{n-2}, w_{n-1}\right)}\left[1+\max _{w_{n} \succ w_{n-1}} \frac{\lambda_{1}^{*}\left(w_{n-1}, w_{n}\right)}{m\left(w_{n-1}, w_{n}\right)}+\max _{w_{n} \succ w_{n-1}} \frac{\lambda_{1}^{*}\left(w_{n-1}, w_{n}\right)}{m\left(w_{n-1}, w_{n}\right)} c_{1}^{*}\left(w_{n}\right)\right] \\
\leq & \max _{w_{n-1} \succ w_{n-2}} \frac{\lambda_{2}^{*}\left(w_{n-2}, w_{n-1}\right)}{m\left(w_{n-2}, w_{n-1}\right)}\left[1+\max _{w_{n} \succ w_{n-1}} \frac{\lambda_{1}^{*}\left(w_{n-1}, w_{n}\right)}{m\left(w_{n-1}, w_{n}\right)}\right] \\
+ & \max _{w_{n-1} \succ w_{n-2}} \frac{\lambda_{2}^{*}\left(w_{n-2}, w_{n-1}\right)}{m\left(w_{n-2}, w_{n-1}\right)} \max _{w_{n} \succ w_{n-1}} \frac{\lambda_{1}^{*}\left(w_{n-1}, w_{n}\right)}{m\left(w_{n-1}, w_{n}\right)} c_{1}^{*}\left(w_{n}\right) .
\end{aligned}
$$

Solving (B.12) in this recursive fashion and using (B.11) we obtain for all $n$ and $w_{0} \in \overline{\mathbb{W}}$

$$
J_{n+1}\left(w_{0}\right) \leq J_{n}\left(w_{0}\right)+\max _{w_{1} \succ w_{0}} \frac{\lambda_{n}^{*}\left(w_{0}, w_{1}\right)}{m\left(w_{0}, w_{1}\right)} \cdots \max _{w_{n} \succ w_{n-1}} \frac{\lambda_{1}^{*}\left(w_{n-1}, w_{n}\right)}{m\left(w_{n-1}, w_{n}\right)} c_{1}^{*}\left(w_{n}\right) .
$$

Using $(\overline{\mathrm{B} .8})$ and $(\overline{\mathrm{B} .10})$ in $(\overline{\mathrm{B} .13})$ we obtain for all $n \in \mathbb{N}$ and $w_{0} \in \overline{\mathbb{W}}$

$$
\begin{aligned}
J_{n+1}\left(w_{0}\right)-J_{n}\left(w_{0}\right) & \leqslant \max _{w_{1} \succ w_{0}} \frac{c_{n}^{*}\left(w_{0}\right)}{1+c_{n-1}^{*}\left(w_{1}\right)} \cdot \max _{w_{2} \succ w_{1}} \frac{c_{n-1}^{*}\left(w_{1}\right)}{1+c_{n-2}^{*}\left(w_{2}\right)} \ldots \max _{w_{n} \succ w_{n-1}} c_{1}^{*}\left(w_{n-1}\right) \cdot c_{1}^{*}\left(w_{n}\right) \\
& =c_{n}^{*}\left(w_{0}\right) \cdot \max _{w_{1} \succ w_{0}} \frac{c_{n-1}^{*}\left(w_{1}\right)}{1+c_{n-1}^{*}\left(w_{1}\right)} \ldots \max _{w_{n-1} \succ w_{n-2}} \frac{c_{1}^{*}\left(w_{n-1}\right)}{1+c_{1}^{*}\left(w_{n-1}\right)} \cdot \max _{w_{n} \succ w_{n-1}} c_{1}^{*}\left(w_{n}\right) .
\end{aligned}
$$

Since $M \geqslant J^{*}(w) \geqslant J_{n}(w)=1+c_{n}^{*}(w)>c_{n}^{*}(w)$ for any $w \in \overline{\mathbb{W}}$ and $n \in \mathbb{N}$, we get

$$
0<J_{n+1}(w)-J_{n}(w) \leqslant M^{2} \cdot\left(\frac{M}{1+M}\right)^{n-1}
$$

for all $w \in \overline{\mathbb{W}}$. But this means that

$$
\left\|J_{n+1}-J_{n}\right\|_{\infty} \leqslant B(\beta)^{n-1}
$$

where $\|\cdot\|_{\infty}$ is the supremum norm on the space of bounded continuous functions on $\overline{\mathbb{W}}$ and $B>0$ and $0<\beta<1$. By standard arguments, this implies

$$
\left\|J_{n+m}-J_{n}\right\|_{\infty} \leqslant B \beta^{n-1} \frac{1}{1-\beta}
$$

for all $n, m>0$ and so $\left(J_{n}\right)_{n \geq 0}$ is a Cauchy sequence, as was to be shown.

Now suppose $m_{A}$ defined in (20) is monotonically increasing. We show that this implies the hypothesis of Lemma B.3. Using the change of variable formula in (B.9) yields

$$
\frac{1}{c_{n}^{*}(w)}=\sum_{w^{\prime} \succ w} \frac{m\left(w, w^{\prime}\right)}{1+c_{n-1}^{*}\left(w^{\prime}\right)} Q\left(w, w^{\prime}\right)=\mathbb{E}_{\nu}\left[\frac{m_{A}(w, \cdot)}{1+c_{n-1}^{*}(W(K(w), \cdot))}\right] .
$$

\footnotetext{
${ }^{19}$ These are $\max _{x}\{A(x)+B(x)\} \leq \max _{x}\{A(x)\}+\max _{x}\{B(x)\}$ and $\max _{x}\{A(x)\} \max _{y \in G(x)}\{B(y)+$ $C(y)\} \leq \max _{x}\{A(x)\} \max _{y \in G(x)}\{B(y)\}+\max _{x}\{A(x)\} \max _{y \in G(x)}\{C(y)\}$ for real-valued functions $A$, $B, C$ and some correspondence $G$.
} 
As the term to the far right is a strictly increasing function whenever $c_{n-1}^{*}$ is decreasing, it follows by induction that each $J_{n}(w)=1+c_{n}^{*}(w), w \in \overline{\mathbb{W}}$ is strictly decreasing which implies $J_{n}(w) \leq J_{n}(\underline{w})$ for all $n$. Taking the limit gives $J^{*}(w) \leq J^{*}(\underline{w})$ for all $w \in \overline{\mathbb{W}}$. Finally, if $A$ is inefficient at $w_{0}$, monotonicity of $J^{*}$ implies $J^{*}\left(w_{0}^{\prime}\right) \leq J^{*}\left(w_{0}\right)<\infty$ also for $w_{0} \geq w_{0}^{\prime}$, i.e., $A$ is also inefficient for all $w_{0} \geq w_{0}^{\prime}$.

\section{B.5 Proof of Lemma 3.2}

For each $w \in \mathbb{W}$ and $\theta \in \Theta$, define $C_{0}^{o}(w, \theta):=K_{0}(w) R\left(K_{0}(w), \theta\right)$ and $\tilde{m}(w):=$ $\mathbb{E}_{\nu}\left[R\left(K_{0}(w), \cdot\right) v^{\prime}\left(C_{0}^{o}(w ; \cdot)\right)\right]$. Using (7), the pricing kernel $m_{0}$ can be written as

$$
m_{0}(w, \theta)=v^{\prime}\left(C_{0}^{o}(w, \theta)\right) / \tilde{m}(w) .
$$

Let $w \in \mathbb{W}$ and $\theta \in \Theta$ be arbitrary but fixed and set $c_{0}:=C_{0}^{o}(w, \theta)$ and $k_{0}:=K_{0}(w)$. Then, by direct computations $\frac{\partial m_{0}}{\partial w}(w, \theta)=\frac{K_{0}^{\prime}(w) v^{\prime}\left(c_{0}\right)}{k_{0} \tilde{m}(w)} H(w)$ where

$$
H(w):=E_{f^{\prime}}\left(k_{0}\right)+\left(1-E_{f^{\prime}}\left(k_{0}\right)\right)\left(\frac{\mathbb{E}_{\nu}\left[R\left(k_{0}, \cdot\right) C_{0}(w, \cdot)\left|v^{\prime \prime}\left(C_{0}(w, \cdot)\right)\right|\right]}{\tilde{m}(w)}-E_{v^{\prime}}\left(c_{0}\right)\right)
$$

determines the sign of $\frac{\partial m_{0}}{\partial w}(w, \theta)$. Using (4), we have $0 \leq E_{v^{\prime}}^{\min } \leq E_{v^{\prime}}\left(c_{0}\right) \leq E_{v^{\prime}}^{\max } \leq 1$ and

$$
E_{v^{\prime}}^{\min } \tilde{m}(w) \leq \mathbb{E}_{\nu}\left[R\left(k_{0}, \cdot\right) C_{0}(w, \cdot)\left|v^{\prime \prime}\left(C_{0}(w, \cdot)\right)\right|\right] \leq E_{v^{\prime}}^{\max } \tilde{m}(w) .
$$

Using these bounds in (B.14), we obtain

$$
H(w) \geq E_{f^{\prime}}\left(k_{0}\right)+E_{v^{\prime}}^{\min }-E_{v^{\prime}}^{\max }-E_{f^{\prime}}\left(k_{0}\right)\left(E_{v^{\prime}}^{\max }-E_{v^{\prime}}^{\min }\right) .
$$

As the r.h.s in (B.15) is non-negative due to (5) in Assumption 3 , the claim follows.

\section{B.6 Proof of Lemma 3.3}

As both $C^{y}$ and $C^{o}$ are continuous, strictly positive functions on their compact domains $\overline{\mathbb{W}}$ and $\overline{\mathbb{W}} \times \Theta$, respectively, we can choose $\bar{\alpha}>0$ such that the 'perturbed' allocation $\left(K, C_{\alpha}^{y}, C_{\alpha}^{o}\right)$ defined as $C_{\alpha}^{y}(w):=C^{y}(w)-\alpha \eta(w)$ and $C_{\alpha}^{o}(w, \theta)=C^{o}(w, \theta)+$ $\alpha \eta(W(K(w), \theta))$ is strictly positive and feasible for all $\alpha \in[-\bar{\alpha}, \bar{\alpha}]$ and $w \in \overline{\mathbb{W}}$. Thus, given $w \in \overline{\mathbb{W}}$, the map $h(\alpha ; w):=u\left(C_{\alpha}^{y}(w)\right)+\mathbb{E}_{\nu}\left[v\left(C_{\alpha}^{o}(w, \cdot)\right)\right]$ is well-defined and determines the utility of a generation born in state $w \in \overline{\mathbb{V}}$ under the perturbation $\alpha \in[-\bar{\alpha}, \bar{\alpha}]$. We will determine $\alpha^{*}>0$ such that $h\left(\alpha^{*} ; w\right)-h(0 ; w)>0$ for all $w \in \overline{\mathbb{W}}$, i.e., the perturbed allocation improves the utility of any generation. Let $w \in \overline{\mathbb{V}}$ be fixed. As $h(\cdot ; w)$ is twice continuously differentiable on the open interval $]-\bar{\alpha}, \bar{\alpha}[$, we have

$$
h(\alpha ; w)-h(0 ; w)=h^{\prime}(0 ; w) \alpha+\frac{1}{2} h^{\prime \prime}(\xi ; w) \alpha^{2}
$$


for $0 \leq \alpha \leq \bar{\alpha}$ and some $0<\xi<\alpha$ that may depend on both $w$ and $\alpha$. By hypothesis,

$$
h^{\prime}(0 ; w)=-u^{\prime}\left(C^{y}(w)\right) \eta(w)+\mathbb{E}_{\nu}\left[v^{\prime}\left(C^{o}(w, \cdot)\right) \eta(W(K(w, \cdot))]>0\right.
$$

for all $w$. Further, using the Lebesgue-dominated convergence theorem

$$
h^{\prime \prime}(\xi ; w)=u^{\prime \prime}\left(C_{\xi}^{y}(w)\right)(\eta(w))^{2}+\mathbb{E}_{\nu}\left[v^{\prime \prime}\left(C_{\xi}^{o}(w, \cdot)\right)(\eta(W(K(w), \cdot)))^{2}\right]<0 .
$$

By the Lebesgue dominated convergence theorem again, both mappings $w \mapsto h^{\prime}(0 ; w)$ and $(\xi ; w) \mapsto h^{\prime \prime}(\xi ; w)$ are continuous on $\overline{\mathbb{W}}$ and $[0, \bar{\alpha}] \times \overline{\mathbb{W}}$, respectively. Thus, there exist $\Delta_{1}>0$ and $\Delta_{2}<0$ such that $h(\alpha ; w)-h(0 ; w) \geqslant \Delta_{1} \alpha+\Delta_{2} \alpha^{2}$ for all $w \in \overline{\mathbb{W}}$ and $\alpha \in[0, \bar{\alpha}]$. Choosing $\alpha^{*}>0$ sufficiently small therefore ensures that $h\left(\alpha^{*} ; w\right)>h(0 ; w)$ for all $w \in \overline{\mathbb{W}}$.

\section{References}

Aiyagari, R. \& D. Peled (1991): "Dominant Root Characterization of Pareto Optimality and the Existence of Optimal Equilibria in Stochastic Overlapping Generations Models", Journal of Economic Theory, 54, 69-83.

Aliprantis, C. D. \& K. C. Border (2007): Infinite Dimensional Analysis. SpringerVerlag, Berlin a.o.

Ball, L., D. Elmendorf \& N. Mankiw (1998): "The Deficit Gamble", Journal of Money, Credit, and Banking, 30, 699-720.

Barbie, M., M. Hagedorn \& A. Kaul (2006): "On the Interaction between Risk Sharing and Capital Accumulation in a Stochastic OLG Model with Production", Working paper.

(2007): "On the Interaction between Risk Sharing and Capital Accumulation in a Stochastic OLG Model with Production", Journal of Economic Theory, 137, $568-579$.

Barbie, M. \& A. Kaul (2009): "The Zilcha criteria for dynamic inefficiency reconsidered", Economic Theory, 40, 339-348.

(2015): "Pareto Optimality and Existence of Monetary Equilibria in a Stochastic OLG Model: A Recursive Appproach", Discussion paper

Buchanan, H. E. \& T. H. Hildebrandt (1908): "Note on the Convergence of a Sequence of Functions of a Certain Type", Annals of Mathematics, 9(3), 123-126. 
Cass, D. (1972): "On Capital Overaccumulation in the Aggregative Neoclassical Model of Economic Growth: A Complete Characterization", Journal of Economic Theory, 4, 200-223.

Chattopadhyay, S. \& P. Gottardi (1999): "Stochastic OLG Models, Market Structure and Optimality", Journal of Economic Theory, 89, 21-67.

Coleman, W. J. I. (1991): "Equilibrium in a Production Economy with an Income Tax", Econometrica, 59, 1091-1104.

Demange, G. \& G. Laroque (2000): "Social Security, Optimality, and Equilibria in a Stochastic Overlapping Generations Economy", Journal of Public Economic Theory, 2(1), 1-23.

Diamond, P. (1965): "National Debt in a Neoclassical Growth Model", American Economic Review, 55(5), 1126-1150.

Farhi, E. \& J. Tirole (2012): "Bubbly Liquidity", Review of Economic Studies, 79, $678-706$.

Galor, O. \& H. E. Ryder (1989): "Existence, Uniqueness, and Stability of Equilibrium in an Overlapping-Generations Model with Productive Capital", Journal of Economic Theory, 49, 360-375.

Gottardi, P. \& F. Kübler (2011): "Social Security and Risk Sharing", Journal of Economic Theory, 146, 1078-1106.

Greenwood, J. \& G. Huffman (1995): "On the Existence of Nonoptimal Equilibria in Dynamic Stochastic Economies", Journal of Economic Theory, 65, 611-623.

Hauenschild, N. (2002): "Capital Accumulation in a Stochastic Overlapping Generations Model with Social Security", Journal of Economic Theory, 106, 201-216.

Hellwig, C. \& G. Lorenzoni (2009): "Bubbles and Self-Enforcing Debt", Econometrica, 77(4), 1137-1164.

Hillebrand, M. (2011): "On the Role of Labor Supply for the Optimal Size of Social Security", Journal of Economic Dynamics and Control, 35, 1091-1105.

(2014): "Uniqueness of Markov Equilibrium in Stochastic OLG Models with Nonclassical Production", Economics Letters, 123(2), 171-176.

Ikeda, D. \& T. Phan (2016): "Toxic asset bubbles", Economic Theory, 61, 241-271.

Kaminigashi, T. \& J. Stachurski (2014): "Stochastic Stability in Monotone Economies", Theoretical Economics, 9, 383 - 407. 
Konishi, H. \& F. Perera-Tallo (1997): "Existence of Steady-State Equilibrium in an Overlapping-Generations Model with Production", Economic Theory, 9, 529-537.

Kübler, F. \& H. Polemarchakis (2004): "Stationary Markov Equilibria for Overlapping Generations", Economic Theory, 24(3), 623-643.

LI, J. \& S. LiN (2012): "Existence and Uniqueness of Steady State Equilibrium in a Generalized Overlapping Generations Model", Macroeconomic Dynamics, 16, 299311.

Magill, M. \& M. Quinzi (2003): "Nonshiftable Capital, Affine Price Expectations and Convergence to the Golden Rule", Journal of Mathematical Economics, 39, 239272 .

Manuelli, R. (1990): "Existence and Optimality of Currency Equilibrium in Stochastic Overlapping Generations Models: The Pure Endowment Case", Journal of Economic Theory, 51, 268-294.

Martin, A. \& J. Ventura (2012): "Economic Growth with Bubbles", American Economic Review, 102, 3033-3058.

McGovern, J., O. F. Morand \& K. L. Reffett (2013): "Computing minimal state space recursive equilibrium in OLG models with stochastic production", Economic Theory, 54, 623-674.

Miao, J., P. Wang \& L. Xu (2016): "Stock market bubbles and unemployment", Economic Theory, 61, 273-307.

Michel, P. \& B. Wigniolle (2003): "Temporary Bubbles", Journal of Economic Theory, 112, 173-183.

Morand, O. F. \& K. L. Reffett (2003): "Existence and uniqueness of equilibrium in nonoptimal unbounded infinite horizon economies", Journal of Monetary Economics, 50, 1351-1373.

(2007): "Stationary Markovian Equilibrium in Overlapping Generations Models with Stochastic Nonclassical Production and Markov Shocks", Journal of Mathematical Economics, 43, 501-522.

Rangazas, P. \& S. Russell (2005): "The Zilcha criterion for dynamic inefficiency", Economic Theory, 26, 701-716.

Tirole, J. (1985): "Asset Bubbles and Overlapping Generations", Econometrica, 53(6), 1499-1528. 
WANG, Y. (1993): "Stationary Equilibria in an Overlapping Generations Economy with Stochastic Production", Journal of Economic Theory, 61(2), 423-435.

WAng, Y. (1994): "Stationary Markov Equilibria in an OLG Model with Correlated Production Shocks", International Economic Review, 35(3), 731-744.

WEIL, P. (1987): "Confidence and the real value of money in an overlapping generations economy", Quarterly Journal of Economics, 102, 1-22.

Zilcha, I. (1990): "Dynamic Efficiency in Overlapping Generations Models with Stochastic Production", Journal of Economic Theory, 52(2), 364-379. 